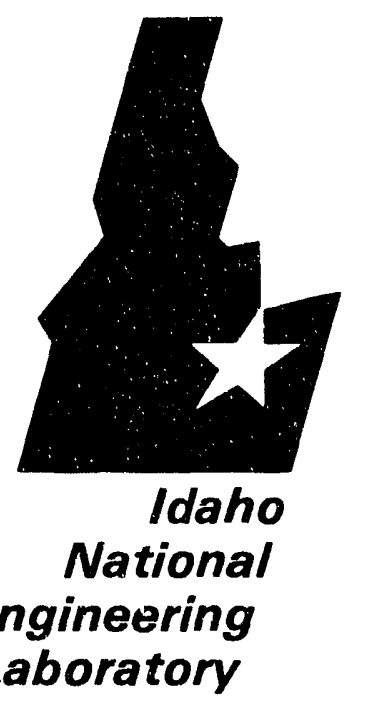

EGG-EAST-9878

JuTy 1992

\section{INFORMAL REPORT}

THEORY AND INPUT REQUIREMENTS FOR THE MULTIDIMENSIONAL COMPONENT IN RELAP5 FOR SAVANNAH RIVER SITE THERMAL HYDRAULIC ANALYSIS

Contributing Authors:

K. E. Carlson

R. A. Riemke

R. J. Wagner

Editors:

R. G. Hanson

E. C. Johnson

\title{
¿SEERE Idaho
}

Work performed under
DOE Contract No. DE.AC07-76IDO1570 
EGG-EAST-9878

July 1992

EGG-EASTL-9878

DE93 001926

INFORMAL REPORT

THEORY AND INPUT REQUIREMENTS FOR THE MULTIDIMENSIONAL COMPONENT IN RELAP5 FOR SAVANNAH RIVER SITE THERMAL HYDRAULIC ANALYSIS

Contributing Authors:
K. E. Carison
R. A. Riemke
R. J. Wagner

Editors:

R. G. Hanson

E. C. Johnson

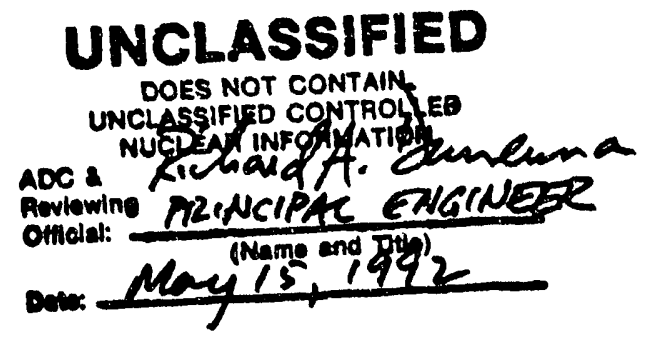


This report was prepared as an account of work sponsored by an agency of the United States Government. Neither the United States Gnvernment nor any agency thereof, nor any of their employees, makes any warranty, express or implied, or assumes any legal liability or responsibility for the accuracy, completeness, or usefulness of any information, apparatus, product, or process disclosed, or represents that its use would not infringe privately owned rights. Reference herein to any specific commercial product, process, or service by trade name, trademark, manufacturer, or otherwise does not necessarily constitute or imply its endorsement, recommendation, or favoring by the United States Government or any agency thereof. The views and opinions of authors expressed herein do not necessarily state or reflect those of the United States Government or any agency thereof.

\title{
THEORY AND INPUT REQUIREMENTS FOR THE MULTIDIMENSIONAL COMPONENT IN RELAP5 FOR SAVANNAH RIVER SITE THERMAL HYDRAULIC ANALYSIS
}

\author{
Contributing Authors: \\ K. E. Carlson \\ R. A. Riemke \\ R. J. Wagner
}

Editors:

R. G. Hanson

E. C. Johnson

July 1992

Idaho National Engineering Laboratory

EG\&G Idaho, Inc.

Idaho Falls, Idaho 83415

Prepared for the U. S. Department of Energy

Assistant Secretary for Defense Programs, 


\section{ABSTRACT}

This report documents the theory and input requirements for the multidimensional component in RELAP5/MOD2.5, Version $3 w$. The equations in Cartesian and cylindrical coordinates are presented as well as the shallow water terms. The implementation of these equations is then discussed. Finally, the constitutive models and input requirements are then described. 


\section{SUMMARY}

The RELAP5/MOD2.5 code is based on a nonhomogeneous and nonequilibrium model for the two-phase system that is solved by a fast, partially implicit numerical scheme to permit economical calculation of system transients. The objective of the RELAP5 development effort from the outset was to produce a code that includes important first-order effects necessary for accurate prediction of system transients but is sufficiently simple and cost-effective such that parametric or sensitivity studies are possible.

This report provides the theory and input requirements for the multidimensional component in RELAP5/MOD2.5, Version 3w. Multidimensional two-phase flow equations are presented as local partial differential equations and in control volume form for mass, momentum, and internal energy. The discrete form of these equations is presented for both Cartesian coordinates and cylindrical coordinates. The implementation of these equations into RELAP5/MOD2.5 is discussed, along with special cases for interior boundaries and 1-D connectiors as well as programming considerations. The constitutive models used for the multidimensional component are presented, which include flow regimes, interphase friction, virtual mass, interphase heat transfer, wall friction, wall heat transfer regimes, and wall heat transfer. The input requirements are presented in the form of card numbers and appropriate input words. Finally, modeling guidelines and assumptions are presented. 


\section{ACKNOWLEDGMENTS}

Development of a complex computer code such as RELAP5 is the result of a team effort. In addition to the code developers who developed and modified the code for the multidimensional component, acknowledgment is made to users Mr. C. B. Davis, Ms. C. Y. Chou, and Mr. R. P. Mart in who were part of the developmental assessment team and were instrumental in finding code errors and supplying alternative methods. Acknowledgment is made to Dr. V. H. Ransom (Purdue University) and Dr. J. A. Trapp (University of Colorado) for their expert consultation, guidance, and equation development on this project, as well as on the ATHENA multidimensional code development, which provided the basis for this project. Acknowledgment is also made to Mr. I. Parzer (Jozef Stefan Institute), who initiated the multidimensional database modifications to RELAP5 before this project began, as well as the volume velocities and elevation checker changes. Acknowledgment is made to Ms. E. C. Johnson for her work in preparing the text in this report and to Ms. G. S. Reilly and Ms. C. E. White for their work in preparing the figures in this report.

The RELAP5 program is indebted to Dr. R. A. Dimenna (Westinghouse Savannah River) for providing technical direction, coordination, and funding for this effort. The comments of Dr. Dimenna, Mr. G. A. Taylor (Westinghouse Savannah River), Mr. C. B. Davis, and Mr. P. E. Murray have significantly improved the accuracy and quality of this report. The program is also indebted to the EG\&G Idaho, Inc. managers during the development and assessment of this capability who provided coordination, direction, and resources: Mr. P. D. Wheatley, Mr. R. Hanson, Dr. C. M. Allison, Mr. G. A. Berna, and Mr. G. W. Johnsen. Finally, the program is indebted to the technical monitors responsible for directing the overall program:

Mr. C. Noble and Mr. W. H. Rettig of the Department of Energy Idaho Field office. 


\section{CONTENTS}

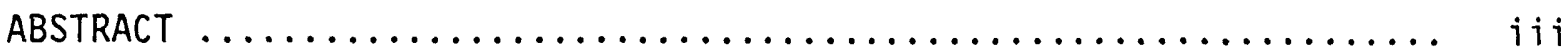

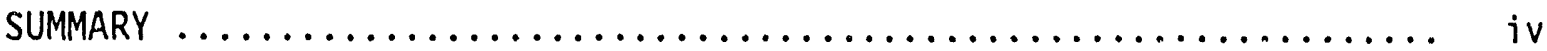

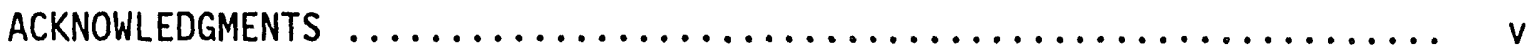

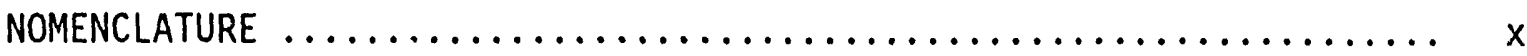

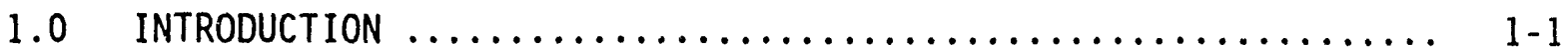

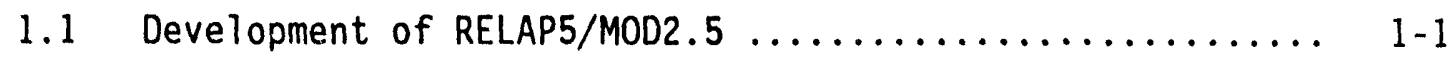

1.2 RELAP5/MOD2.5 Application to Savannah River Site ....... 1-2

1.3 References ................................ 1-3

2.0 MULTIDIMENSIONAL TWO-PHASE FIJW EQUATIONS IN CARTESIAN AND

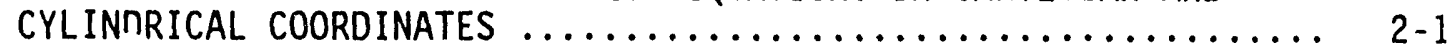

2.1 Mass and Internal Energy Equations ............... 2-1

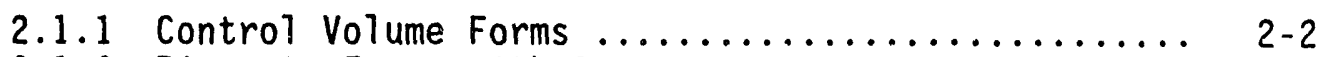

2.1.2 Discrete Forms with Approximations ............. 2-4

2.1.2.1 Cartesian Coordinates ................ 2-8

2.1.2.2 Cylindrical Coordinates $\ldots \ldots \ldots \ldots \ldots \ldots, 2-10$

2.2 Momentum Equations $\ldots \ldots \ldots \ldots \ldots \ldots \ldots \ldots \ldots \ldots \ldots .2-10$

2.2.1 Control Volume Forms .................. 2-12

2.2.2 Discrete Forms with Approximations ............. 2-13

2.2.2.1 Cartesian Coordinates .............. 2-13

2.2.2.2 Cylindrical Coordinates ............ 2-14

2.2 .3 Shallow Water Equations ..................... 2-14

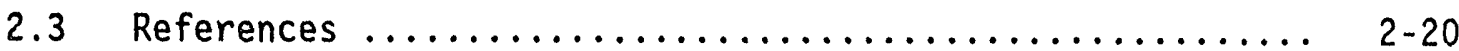

3.0 IMPLEMENTATION OF THE MULTIDIMENSIONAL MODEL TWO-PHASE FLOW

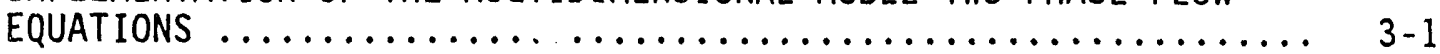

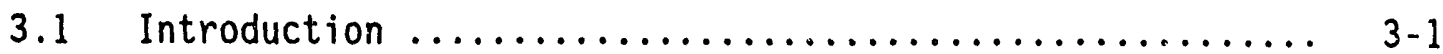

3.2 Cartesian Coordinates ........................... 3-1

3.2.1 Momentum Flux Terms ..................... $3-2$

3.2 .2 Terms at Interior Boundaries .................. $3-11$

3.2.2.1 Side Cells ....................... 3-11

3.2.2.2 Corner Cells ..................... 3-13

3.2 .3 Terms for $1-D$ Connections ..................... $3-19$

3.2.3.1 Pipe Connections .................... 3-19

3.2.3.2 Branch Connections .................. $3-22$ 
3.3 Cylindrical Coordinates ................... 3-27

3.3.1 Momentum Flux Terms ................... 3-27

3.3.2 Terms at Interior Boundaries ................ 3-36

3.3.3 Terms for 1-D Connections .................. 3-41

3.4 Programming Considerations $\ldots \ldots \ldots \ldots \ldots \ldots \ldots \ldots . \ldots . \ldots . \ldots 3$

3.5 Routines ................................ $3-44$

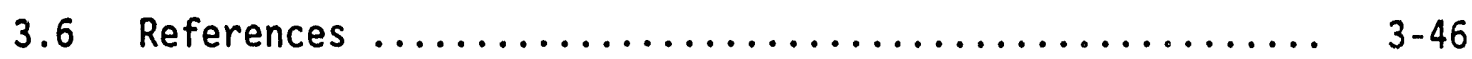

4.0 MULTIDIMENSIONAL CONSTITUTIVE MODELS $\ldots \ldots \ldots \ldots \ldots \ldots \ldots \ldots \ldots$

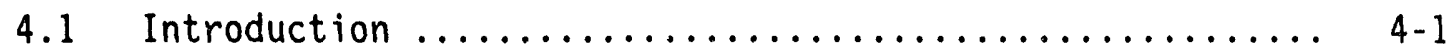

4.2 Flow Regimes $\ldots \ldots \ldots \ldots \ldots \ldots \ldots \ldots \ldots \ldots \ldots \ldots \ldots \ldots$ 4-1

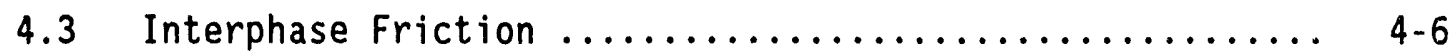

4.4 Virtual Mass $\ldots \ldots \ldots \ldots \ldots \ldots \ldots \ldots \ldots \ldots \ldots \ldots \ldots \ldots$ 4-8

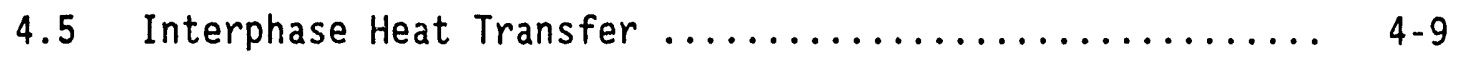

4.6 Wall Friction .............................. 4-10

4.7 Wall Heat Transfer Regimes ................... 4-13

4.8 Wall Heat Transfer ....................... 4.13

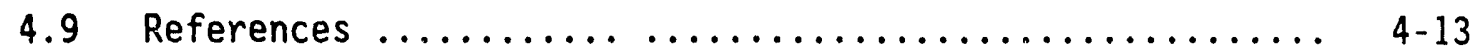

5.0 MULTIDIMENSIONAL INPUT REQUIREMENTS $\ldots \ldots \ldots \ldots \ldots \ldots \ldots \ldots \ldots \ldots$

5.1 Card CCCO001, MULTID Information Card ............. 5-2

5.2 Card CCCOO02, Rotation Angle Data Card ............ 5-3

5.3 Cards CCCOXnN, Mesh Interval Cards .............. 5-5

5.4 Cards CCCINnN, Volume Option Cards ............. 5-6

5.5 Cards CCC2NNN, Volume Friction Data Cards .......... 5-8

5.6 Cards CCC3001 Through CCC5999, Junction Data Cards .... 5-9

5.7 Cards CCC6nNN, Volume Initial Condition $\mathrm{Da}^{+} \mathrm{a}$ Cards ..... 5-11

5.8 Cards CCC7001 Through CCC9999, Junction Initial

Condition Data Cards ......................... 5-14

6.0 MODELING GUIDELINES AND ASSUMPTIONS $\ldots \ldots \ldots \ldots \ldots \ldots \ldots \ldots \ldots$ 


\section{FIGURES}

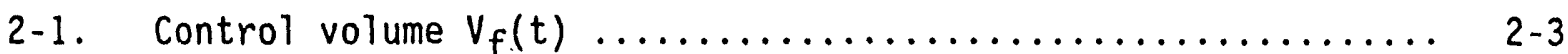

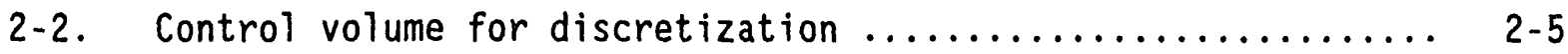

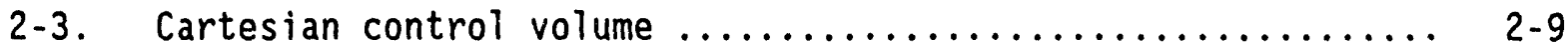

2-4. Cylindrical control volume $\ldots \ldots \ldots \ldots \ldots \ldots \ldots \ldots \ldots \ldots \ldots \ldots \ldots \ldots \ldots, 2-11$

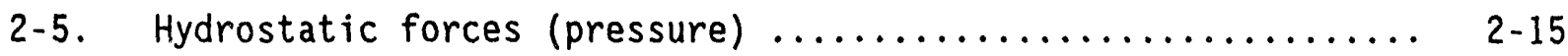

2-6. Force on gas due to pressure distribution $\ldots \ldots \ldots \ldots \ldots \ldots \ldots .16$

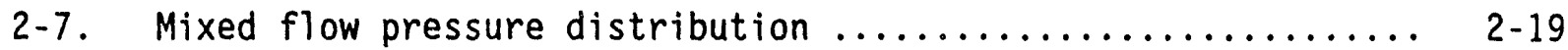

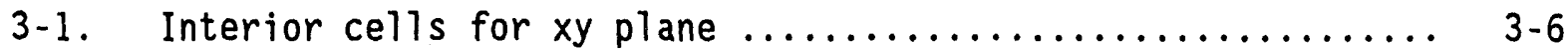

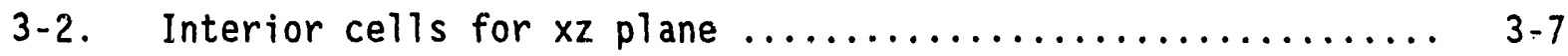

3-3. Control volume for the momentum cell absut $v_{x_{i+1 / 2}, j, k} \ldots \ldots .3-8$

3-4. Control volume for the momentum cell about $v_{y_{i} j+1 / 2, k} \ldots \ldots .3-9$

3-5. Control volume for the momentum cell about $v_{z_{j, j}, k+1 / 2} \ldots \ldots$ 3-10

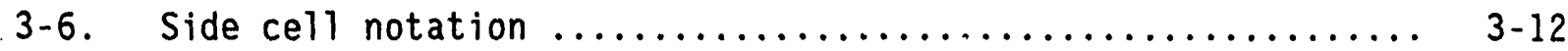

3-7. Interior, side, and corner cells ..................... 34

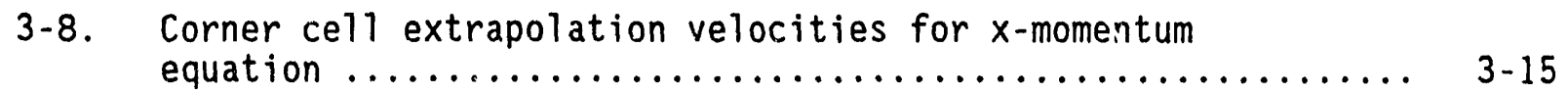

3-9. Corner cell extrapolation velocities for $y$-momentum equation ....................................... 3-17

3-10. Necessary extrapolations ............................ 3-.

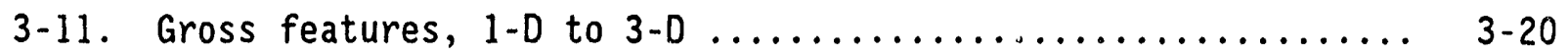

3-12. Axial velocity profile, expanding flow ................ 3-21

3-13. Velocities needed for junctions 3,5 , and $7 \ldots \ldots \ldots \ldots \ldots .3-23$

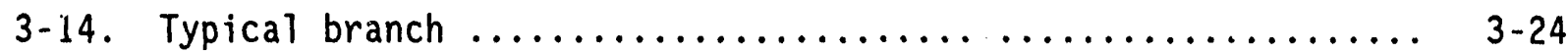

3-15. Extrapolated velocities for a plenum $\ldots \ldots \ldots \ldots \ldots \ldots \ldots \ldots$ 3-26

3-16. Control volume for the momentum cell about $v_{r_{i+1 / 2, j, k}} \ldots \ldots .3-29$

3-17. Control volume for Bernoulli correction ............... 3-31

3-18. Control volume for the momentum cell about $v_{\theta_{i, j+1 / 2, k} \ldots \ldots}, \ldots-34$ viii 


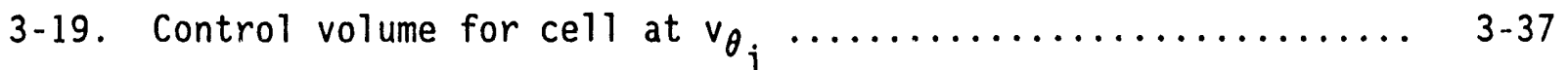

3-20. Control volume for radial momentum flux $\ldots \ldots \ldots \ldots \ldots \ldots \ldots . . . .39$

3-21. Control volume for azimuthal momentum flux $\ldots \ldots \ldots \ldots \ldots \ldots \ldots$ 3-40

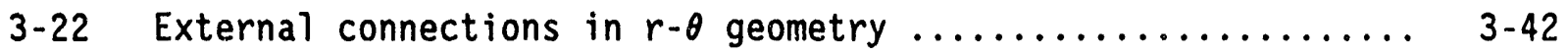

4-1. Horizontal flow map and flow regimes $\ldots \ldots \ldots \ldots \ldots \ldots \ldots . \ldots . \ldots . \ldots$

4-2. Vertical/annular flow map and flow regimes $\ldots \ldots \ldots \ldots \ldots \ldots . . .4$

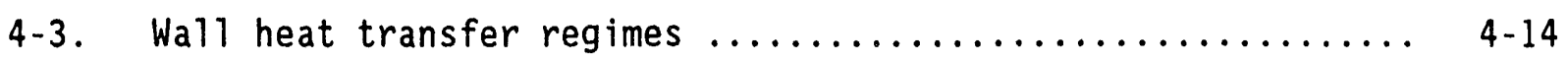




\section{NOMENCLATURE}

A

a

$A_{j}$

$A_{c i}$

b

D

$D_{S}$

FAAJ

FAAV

FIJ

FIVOL

FWFJ

FWGJ

FWFVOL

FWGVOL

f

fvcahs

G

g

HIF

HIG

h

NX

NY

NZ

$n$

Area

Constant in wall friction correlation

Total area of face $i$ for volume $V$

Area of $A_{j}$ for flowing fluid phase under consideration

Constant in wall friction correlation

Increment of length, volume hydraulic diameter

Viscous dissipation rate per unit volume

Junction virtual mass coefficient

Volume virtual mass coefficient

Junction interphase drag coefficient

Volume interphase drag coefficient

Junction wall friction coefficient for liquid

Junction wall friction coefficient for vapor

Volume wall friction coefficient for liquid

Volume wall friction coefficient for vapor

Vector field, external forces per unit mass

Junction $f l a g$

Volume mass flux

Gravitaticnal acceleration

Volume interphase heat transfer coefficient for liquid

Volume interphase heat transfer coefficient for vapor

Enthalpy, height

Number of intervals in the $x$ or $r$ coordinate direction

Number of intervals in the $y$ or $\theta$ coordinate direction

Number of intervals in the $z$ coordinate direction

Outwardly directed unit normal to a surface 


\begin{tabular}{|c|c|}
\hline$P$ & Pressure \\
\hline pvbfe & Volume flag \\
\hline$Q$ & Rate of direct heating per unit mass \\
\hline$Q_{i}$ & $\begin{array}{l}\text { Rate of heat addition to the phase per unit volume at the } \\
\text { interface }\end{array}$ \\
\hline q & Rate of contact heat addition per unit area \\
\hline $\mathrm{R}$ & Rate of direct heating per unit volume \\
\hline r & Spatial coordinate (cylindrical) \\
\hline$S$ & Viscous stress tensor \\
\hline$s_{c}$ & Fixed surface of interest \\
\hline$s_{i}$ & $\begin{array}{l}\text { Interface surface between the phase in } V_{f} \text { and the other } \\
\text { phase }\end{array}$ \\
\hline $\mathrm{T}$ & Temperature \\
\hline$t$ & Time \\
\hline$x$ & Spatial coordinate (Cartesian) \\
\hline u & Specific internal energy \\
\hline V & Total volume of both phases \\
\hline$v_{f}$ & Volume of phase of interest \\
\hline v & Velocity \\
\hline$v_{\text {in }}$ & Normal component of $v$ on face $i$ \\
\hline$v_{S}$ & Velocity of surface $s$ \\
\hline$x$ & Quality, position number in direction 1 \\
\hline$x_{B}$ & Bernoulli correction factor \\
\hline$x$ & Spatial coordinate (Cartesian) \\
\hline YY & Position number in direction 2 \\
\hline y & Spatial coordinate (Cartesian) \\
\hline z & Position number in direction 3 \\
\hline $\mathrm{ZZ}$ & Spatial coordinate (Cartesian and cylindrical), elevation \\
\hline
\end{tabular}




\section{Greek Symbols}

$\alpha$

B

CHF

f

g

I

i

j

K

k

L

M

m

n

0

p

Q

$r$
Void fraction

Mass generation rate per unit volume

Angular coordinate (cylindrical), face $\theta$

Viscosity

Density

Viscous stress gradient

Scalar field, elevation angle

\section{SUbSCRIPTS}

Bottom

Critical heat flux

Liquid phase

Vapor phase

Inte: ?ace

Face $i$, index for $x$ or $r$

Index for $y$ or $\theta$

Index for cell $\mathrm{K}$

Index for $z$

Index for cell L

Index for cell $M$

Mean value

Noncondensable

Index for cell 0

Index for cell $p$

Index for cell $Q$

Face $r$ 


\begin{tabular}{ll} 
s & Surface, steam \\
sat & Saturation \\
$v$ & Top \\
w & Volume v \\
wall & Wall \\
$x$ & Wall \\
$y$ & Face $x$ \\
$z$ & Face $y$ \\
1 & Face z \\
2 & Direction 1 \\
3 & Direction 2 \\
\hline
\end{tabular}

\section{SUPERSCRIPTS}

$\begin{array}{ll}c & \text { Corner cell } \\ i & \text { Interior cell } \\ \mathrm{s}, \mathrm{n}+1 & \text { Time level index } \\ & \text { Side cell } \\ & \\ + & \text { Velocity index } \\ \nabla & \text { Vector, average value, velocity index } \\ \Delta & \text { "Del, or gradient, operator }\end{array}$




\title{
THEORY AND INPUT REQUIREMENTS FOR
}

\section{THE MULTIDIMENSIONAL COMPONENT IN}

\author{
RELAP5 FOR SAVANNAH RIVER SITE
}

\section{THERMAL HYDRAULIC ANALYSIS}

\subsection{INTRODUCTION}

The RELAP5 computer code is a light water reactor transient analys is code developed for the U.S. Nuclear Regulatory Commission (NRC) for lise in rulemaking, licensing audit calculations, evaluation of operator guidelines, and as a basis for a nuclear plant analyzer. Specific applications of this capability have included simulations of transients in LWR systems, such as loss of coolant accidents (LOCA), anticipated transients without scram (ATWS), and operational transients such as loss of feedwater, loss of offsite power, station blackout, and turbine trip. RELAP5 is a highly generic code that, in addition to calculating the behavior of a reactor coolant system during a transient, can be used for simulation of a wide variety of hydraulic and thermal transients in both nuclear and nonnuclear systems involving steam-water noncondensable solute fluid mixtures.

\subsection{DeVelopment OF RELAP5/MOD2.5}

RELAP5/MOD2.5 was produced by improving and extending the modeling base of RELAP5/MOD2. ${ }^{1-1,2,3}$ There is no official stand-alone RELAP5/MOD2.5 documentation, as it is an interim version in the development of RELAP5/MOD3. The models in RELAP5/MOD2.5 are documented in the SCDAP/RELAP5/MOD2 documentation. ${ }^{1-4}$ The new models include:

- the Bankoff counter-current flow limiting correlation, which is based on actual geometry and can be activated by the user at each junction in the system model. 
Improvements to existing models include:

- the Bestion correlation for interfacial friction for bundle types of geometry in the bubbly-slug flow regime in vertical flow passages

Additional user conveniences include:

- code speedup through vectorization for the CRAY/X-MP computer

- code portability through the conversion of the FORTRAN coding to adhere to the FORTRAN 77 standard

- code execution and validation on a variety of systems [CRAY/X-MP, CYBER (NOS/VE), IBM 3090 (MVS), VAX (ULTRIX), and DECworkstation (ULTRIX)].

\subsection{RELAP5/MOD2.5 APPLICATION to Savannah River Site}

Although originally developed for NRC, RELAP5/MOD2.5 has been used to model Department of Energy (DOE) reactors, including Savannah River, 1-5,6,7 $\mathrm{N}$-Reactor, 1-8 and the Advanced Test Reactor (ATR). 1-9 The Savannah River applications have included models of the K-reactor and $L$-reactor. Both the water plenum and the moderator tank in the reactor vessel of these reactors are currently being modeled using the code's cross-flow option to approximate the multidimensional features of the flow. The multidimensional component in RELAP5 was developed to allow the user to model more accurately the multidimensional features of the water plenum and the moderator tank. The multidimensional component (indicated by MULTID in the input cards) defines a one, two, or three dimensional array of volumes and the internal junctions connecting the volumes. The geometry can be either Cartesian $(x, y, z)$ or cylindrical $(r, \theta, z)$. An orthogonal, three-dimensional grid is defined by mesh interval input data in each of the three coordinate directions. The major modifications to the code were to add the full momentum flux terms, as well as the associated input processing and checking. 
This document shows the theory and input requirements for the multidimensional component that is in RELAP5/MOD2.5, Version $3 w$. Topics covered are multidimensional two-phase flow equations in Cartesian and cylindrical coordinates, implementation of these equations into RELAP5/MOD2.5, constitutive models, input requirements, and modeling guidelines/assumptions.

\subsection{References}

1-1. V. H. iansom et a7., RELA,05/MOD2 Coce Marus?, Volumes 1 and 2, NUREG/CR-4312, EGG-2396, Revision 1, March 1987.

1-2. V. H. Ransom et al., RELAP5/MOD2 Code Manual, Volume 3:

Developmental Assessment Problems, EGG-TFM-7952, December 1987.

1-3. R. A. Dimenna et al., RELAP5/MOD2 Models and Correlations, NUREG/CR-5194, EGG-2531, August 1988.

1-4. C. M. Allison et al., SCDAP/RELAP5/MOD2 Code Manual, Volumes 1, 2 and 3, NUREG/CR-5273, EGG-2555, September 1989.

1-5. C. B. Davis et a1., Benchmarking the RELAP5 L-Reactor Model with Savannah River Test Data, EGG-EAST-8336, Apri1 1989.

1-6. R. A. Shaw, Preliminary Benchmarking of the Hexagonal RELAP5 L-Reactor Model With Savannah River Test Data, EGG-EAST-8774, December 1989.

1-7. C. Y. Chou and C. B. Davis, Single-Phase RELAP5 Benchmarks With 1989 L-Area Test Data, EGG-EAST-9440, January 1991.

1-8. M. A. Bolander and C. D. Fletcher, Simulation of Cold Leg Manifold Break and Station Blackout Sequences in the N-Reactor, EGG-TFM-7891, February 1988.

1-9. J. D. Burtt et a1., Advanced Test Reactor Large Break Loss-of-Coolant Accident Break Spectrum Study, EGG-TFM-8082, Apri1 1988. 


\subsection{MULTIDIMENSIONAL TWO-PHASE FLOW EQUATIONS IN CARTESIAN AND CYLINDRICAL COORDINATES}

\subsection{Mass and Internal Energy Equations}

The partial differential equations are of the form

$$
\begin{aligned}
& \frac{\partial \rho}{\partial t}+\nabla \cdot(\rho \bar{v})=0 \\
& \left.\rho\left(\frac{\partial U}{\partial t}+\bar{v} \cdot \nabla U\right)=-\nabla \cdot \bar{q}-P \nabla \cdot \bar{v}+\operatorname{tr}(\bar{S} \cdot \nabla \bar{v})+\rho Q \quad \text { (Reference 2-1, } p 304\right) \\
& \text { or using Equation }(2-1) \\
& \frac{\partial(\rho U)}{\partial t}+\nabla \cdot(\rho U \bar{v})=-\nabla \cdot \bar{q}-P \nabla \cdot \bar{v}+\operatorname{tr}(\bar{S} \cdot \nabla \bar{v})+\rho Q
\end{aligned}
$$

In general we will be going back and forth between the local partial differential equation form above and the control volume form derived below. Before deriving the control volume form, it should be noted that Equation (2-1) is in conservative form (a11 derivative terms appear without any coefficients), but Equation (2-2) is not. The total energy equation, including internal and kinetic energies, can be written in conservative form, but the internal energy equation can not.

The local mass equation, because it is in conservative form, could be stated directly in control volume form as a balance between rates of change and surface fluxes. Because the internal energy equation is not a conservative form partial differential equation, no such primitive control volume statement exists. The control volume form of the internal energy equation must be derived by performing a spatial integration of Equation (2-2). When this is done nonconservative terms appear, i.e., not all terms are (a) time derivatives of volume integrals, (b) surface fluxes, or (c) volume sources. 


\subsubsection{Control Volume Forms}

Consider the contrcl volume $V_{f}(t)$ shown in Figure 2-1. $V_{f}(t)$ is the volume occupied by one phase in the region of interest. $V_{f}(t)$ is surrounded by surfaces $S_{j}(t)$ and $S_{c}$. The surface $S_{j}(t)$ is the interface between the phase in $V_{f}(t)$ and the other phase. The surface $S_{C}$ is the fixed surface of interest. The surface $S_{j}(t)$ moves with velocity $\bar{v}_{S}$ whereas $S_{C}$ is fixed, i.e., $\bar{v}_{S}=0$ on $S_{C}$. Note, (a) $\bar{v}_{S}$ is the velocity of the interface, not the fluid velocity $\bar{v}$, and (b) $v_{f}(t)$ is a function of time because $S_{j}(t)$ is moving.

We need the following two integral theorems for any scalar field $\phi$ and vector field $f$ defined in $V_{f}(t)$.

$$
\begin{aligned}
& \frac{\partial}{\partial t} \int_{V_{f}(t)} \phi d V=\int_{V_{f}(t)} \frac{\partial \phi}{\partial t} d V+\int_{S_{j}(t)} \phi \bar{v}_{s} \cdot \bar{n} d A \\
& \int_{V_{f}(t)} \nabla \cdot \bar{f} d V=\int_{S_{j}(t)} \bar{f} \cdot \bar{n} d A+\int_{S_{C}}^{\prime} \bar{f} \cdot \bar{n} d A \quad \text { (Divergence Theorem) }
\end{aligned}
$$

To derive the control volume form of the mass equation integrate Equation (2-1) over $V_{f}(t)$, then apply Equation (2-4) to the gradient terms and use Equation (2-3) to evaluate the time derivative term. The result is

$$
\frac{\partial}{\partial t_{V_{f}}(t)} \int_{S_{C}} \rho d V+\int_{S_{C}} \rho \bar{v} \cdot \bar{n} d A+\int_{S_{j}(t)} \rho\left(\bar{v}-\bar{v}_{S}\right) \cdot \bar{n} d A=0
$$

Equation (2-5) is the general control volume form of the mass conservation equation. It can be applied to any shape control volume, $V$, containing $V_{f}$ to obtain discretized finite difference formulas. In the next section we will do this using Cartesian coordinate control volumes and cylindrical coordinate control volumes.

To derive the control volume form of Equation (2-2), we integrate Equation (2-2) over $V_{f}(t)$ and apply Equation (2-3) and (2-4) as before. The result is 


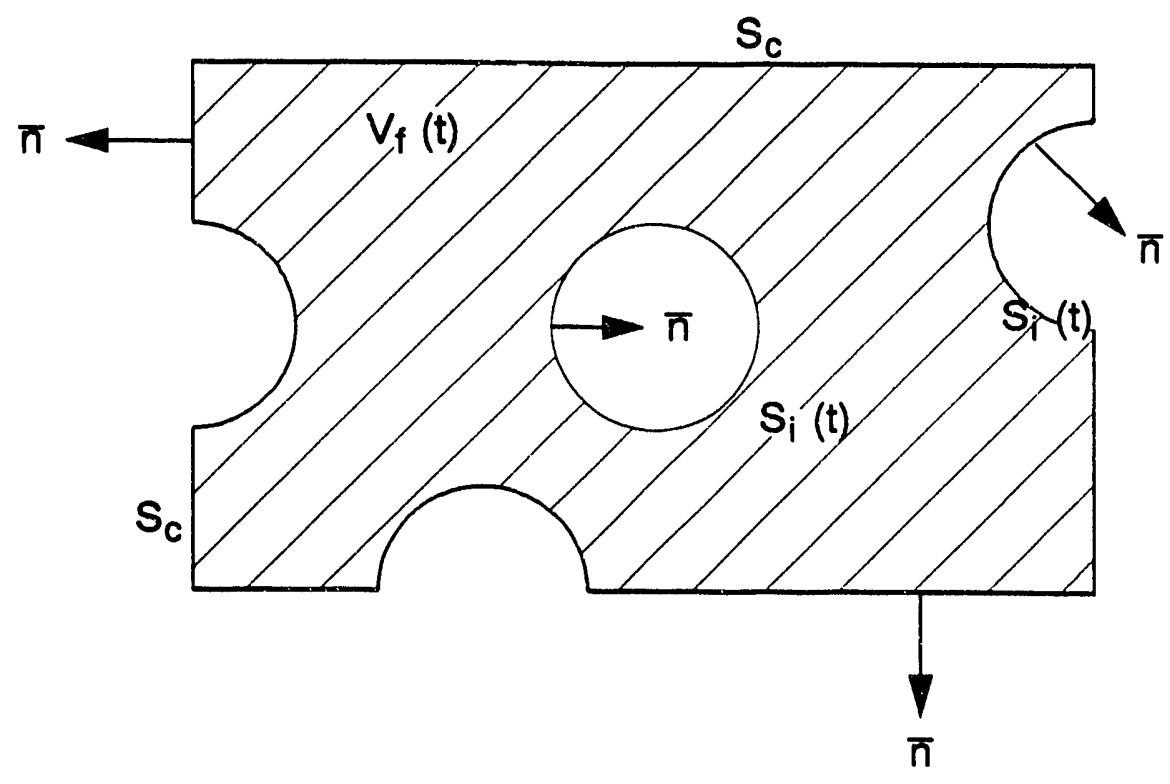

M552 rer-0492-01

Figure 2-1. Control volume $V_{f}(t)$. 


$$
\begin{aligned}
& \frac{\partial}{\partial t} \int_{V_{f}(t)} \rho U d V+\int_{S_{C}} \rho U \bar{v} \cdot \bar{n} d A+\int_{S_{j}(t)}, \bar{U}\left(\bar{v}-\bar{v}_{S}\right) \cdot \bar{n} d A \\
= & -\int_{S_{C}} \bar{q} \cdot \bar{n} d A-\int_{S_{j}(t)} \bar{q} \cdot \bar{n} d A-\int_{V_{f}(t)} P \nabla \cdot \bar{v} d V+\int_{V_{f}(t)} \operatorname{tr}(\bar{S} \cdot \nabla \bar{v}) d V+\int_{V_{f}(t)} \rho Q d V
\end{aligned}
$$

This is the basic control volume form for the internal energy equation.

Before proceeding to the discrete forms we introduce some terminology for the source terms:

$$
\begin{array}{ll}
\Gamma=\frac{1}{V} \int_{S_{i}(t)} \rho\left(\bar{V}_{S}-\bar{v}\right) \cdot \bar{n} d A & =\text { mass generation rate per unit volume } \\
R=\frac{1}{V} \int_{V_{f}(t)} \rho Q d V & =\text { rate of direct heating per unit volume } \\
D_{S}=\frac{1}{V} \int_{V_{f}(t)} \operatorname{tr}(\bar{S} \cdot \nabla \bar{v}) d V & =\text { viscous dissipation rate per unit volume. }
\end{array}
$$

\subsubsection{Discrete Forms With Approximations}

Consider Figure 2-2 with the following terminology:

$$
\begin{array}{ll}
V & =\text { volume in dotted lines, i.e., spatial volume containing } \\
& V_{f} \text { plus "holes" occupied by other phase } \\
A_{i} & =\text { total area of face } i \text { of } V \\
A_{c i} & =\text { area of } A_{i} \text { flowing fluid phase we are considering. }
\end{array}
$$

For any particular face $\alpha_{i}=\frac{A_{c i}}{A_{i}}$ and $\alpha_{v}=\frac{V_{f}}{V}$.

With this terminology, we return to Equations $(2-5)$ and $(2-6)$. 


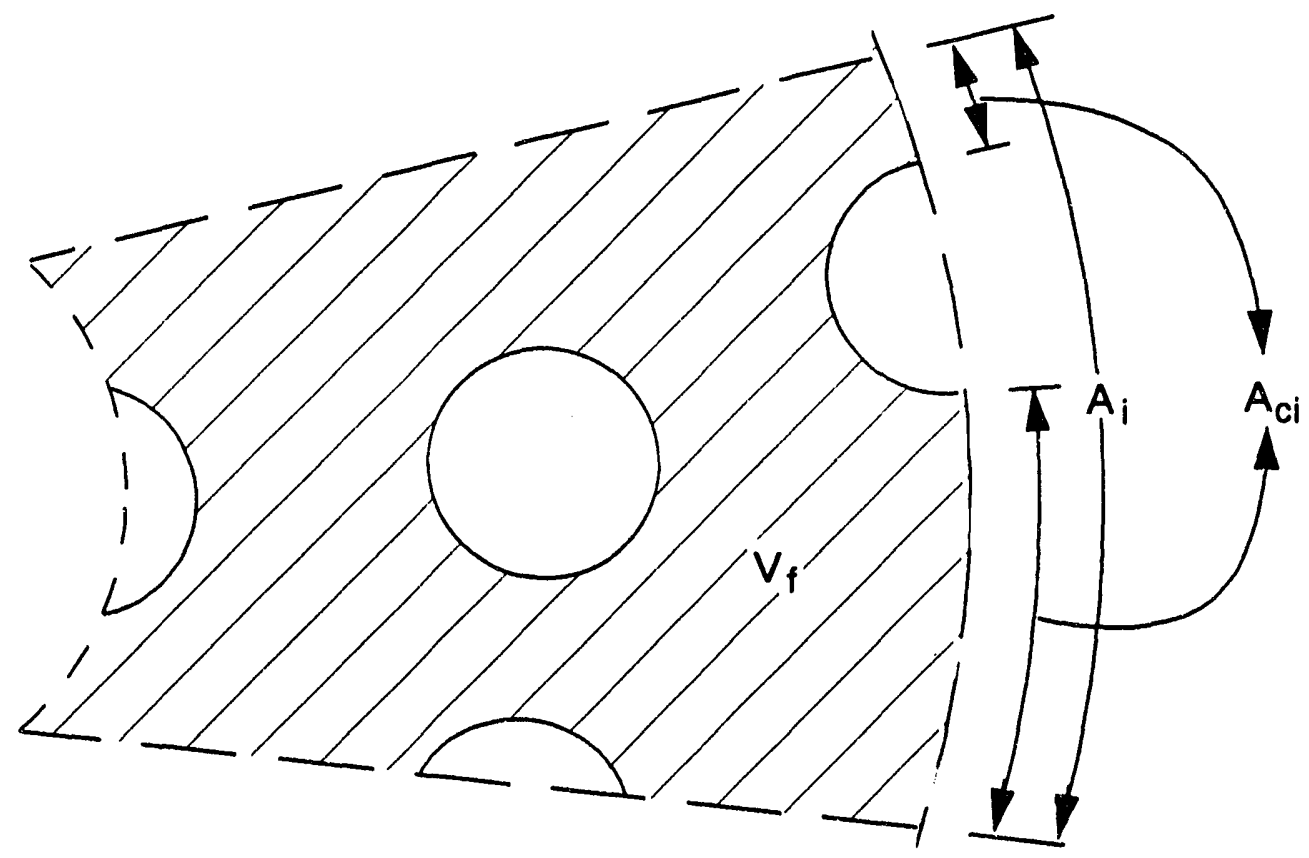

M552 rer-0492-0R

Figure 2-2. Control volume for discretization. 
To discretize Equation (2-5) and (2-6), we will represent all yolume and surface integrals assuming uniform profiles for each variable. Doing this for Equation (2-5) gives

$$
\begin{aligned}
& \frac{\partial}{\partial t}\left(\rho V_{f}\right)+\sum_{i} A_{C i} \rho_{j}(\bar{V} \cdot \bar{n})_{i}=V \Gamma \\
& \text { or } \\
& \frac{\partial}{\partial t}\left(\alpha_{V} \rho V\right)+\sum_{i} A_{i} \alpha_{i} \rho_{i} V_{i n}=V \Gamma
\end{aligned}
$$

whers $v_{\text {in }}$ is the normal component of $\bar{v}$ on face $i$.

To complete the discrete form of the mass equation, the iime derivative in Equation (2-7) is written as

$$
\frac{\partial}{\partial t}\left(\alpha_{v} \rho V\right)=V\left(\rho \frac{\partial \alpha_{v}}{\partial t}+\alpha_{v} \frac{\partial \rho}{\partial t}\right)
$$

and discretized with old $n$-time levels on the coefficients. The flux terms have the normal face velocities evaluated at $n+1$-time levels with the face $\alpha_{i}$ and $\rho_{i}$ donored at the old $n$-time level. $\Gamma$ is linearly implicit as in the present RELAP5.

We now consider Equation (2-6). The third term on the right-hand side of Equation (2-6) is approximated assuming a uniform pressure in the volume $v$. Hence,

$$
\begin{aligned}
& \int_{V_{f}(t)} P \nabla \cdot \bar{v} d V=P \int_{V_{f}(t)} \nabla \cdot \bar{V} d V \\
& =P\left[\int_{S_{C}} \bar{v} \cdot \bar{n} d A+\int_{S_{j}(t)} \bar{V} \cdot \bar{n} d A\right] \quad \text { (using the Divergence Theorem) }
\end{aligned}
$$

or 


$$
\int_{V_{f}(t)} P \nabla \cdot \bar{v} d V=P\left[\int_{S_{C}} \bar{v} \cdot \bar{n} d A+\int_{S_{j}(t)}\left(\bar{v}-\bar{v}_{S}\right) \cdot \bar{n} d A+\int_{S_{j}(t)} \bar{v}_{s} \cdot \bar{n} d A\right]
$$

Now applying Equation $(2-3)$ to $\phi=1$ we have

$$
\frac{\partial}{\partial t} \int_{V_{f}(t)} 1 d V=\int_{S_{j}(t)} \bar{v}_{s} \cdot \bar{n} d A
$$

and using this for the last term in Equation (2-8) gives (remember $P$ is assumed constant)

$$
\int_{V_{f}(t)} P \nabla \cdot \bar{v} d V=P \int_{S_{C}} \bar{v} \cdot \bar{n} d A+P \int_{S_{j}(t)}\left(\bar{v}-\bar{v}_{S}\right) \cdot \bar{n} d A+P \frac{\partial V_{f}}{\partial t}
$$

With this approximation for the pressure-volume change work term, Equation (2-6) becomes

$$
\begin{aligned}
& \frac{\partial}{\partial t} \int_{V_{f}(t)} \rho U d V+\int_{S_{C}} \rho U \bar{v} \cdot \bar{n} d A= \\
& -\int_{S_{C}} \bar{q} \cdot \bar{n} d A-\int_{S_{j}(t)} \bar{q} \cdot \bar{n} d A-P \frac{\partial\left(\alpha_{V} V\right)}{\partial t}-P \int_{S_{C}} \bar{v} \cdot \bar{n} d A-\int_{S_{j}(t)}(U+P / \rho) \rho\left(\bar{V}-V_{S}\right) \cdot \bar{n} d A \\
& +D_{S} V+R V
\end{aligned}
$$

As before, assuming uniform profiles for all integrands, Equation (2-10) can be discretized as

$$
\begin{aligned}
& \frac{\partial}{\partial t}\left(\alpha_{v} \rho U V\right)+\sum_{i} A_{i} \alpha_{i} \rho_{i} U_{i} v_{i n} \\
& =-\int_{S_{C}} \bar{q} \cdot \bar{n} d A+Q_{j} V-P\left[\frac{\partial \alpha_{V} V}{\partial t}+\sum_{i} A_{j} \alpha_{i} v_{i n}\right] \\
& \quad+h_{i} \Gamma V+D_{s} V+R V
\end{aligned}
$$


where

$$
\begin{aligned}
& h_{i}=U+P / \rho \text { on } S_{i} \quad \text { (average or mean value of) } \\
& Q_{i}=-\frac{1}{V} \int_{S_{i}(t)} \bar{q} \cdot \bar{n} d A=\begin{array}{r}
\text { rate of heat addition to phase per unit } \\
\text { volume at the interface. }
\end{array} \\
& -\int_{S_{C}} \bar{q} \cdot \bar{n} d A
\end{aligned}
$$

When applying Equation (2-11) to a finite control volume, the discretization in time is like that in the mass equation and it is writter as

$$
\frac{\partial}{\partial t}\left(\alpha_{V} \rho U V\right)=V\left(\rho U \frac{\partial \alpha_{V}}{\partial t}+\alpha_{V} U \frac{\partial \rho}{\partial t}+\alpha_{V} \rho \frac{\partial U}{\partial t}\right)
$$

The donored flux terms are also like that in the mass equation. [Here they are the second term on the left and the fourth term on the right-hand side of Equation (2-11).] The interface heat transfer, $Q_{j}$, is evaluated in a linearly implicit manner, and $h_{i}$ is at the old time level. The $D_{s}$ and $R$ terms are explicit.

2.1.2.1 Cartesian Coordinates. For a finite Cartesian coordinate control volume as in Figure $2-3$, the volume $V$ is given by

$$
v=\int_{z_{1}}^{z_{2}} \int_{y_{1}}^{y_{2}} \int_{x_{1}}^{x_{2}} d x d y d z=\Delta x \Delta y \Delta z
$$

and for the area terms we have

$$
\begin{array}{ll}
x \text {-faces } & A_{x}=\Delta y \Delta z \\
y \text {-faces } & A_{y}=\Delta x \Delta z \\
z \text {-faces } & A_{z}=\Delta x \Delta y
\end{array}
$$



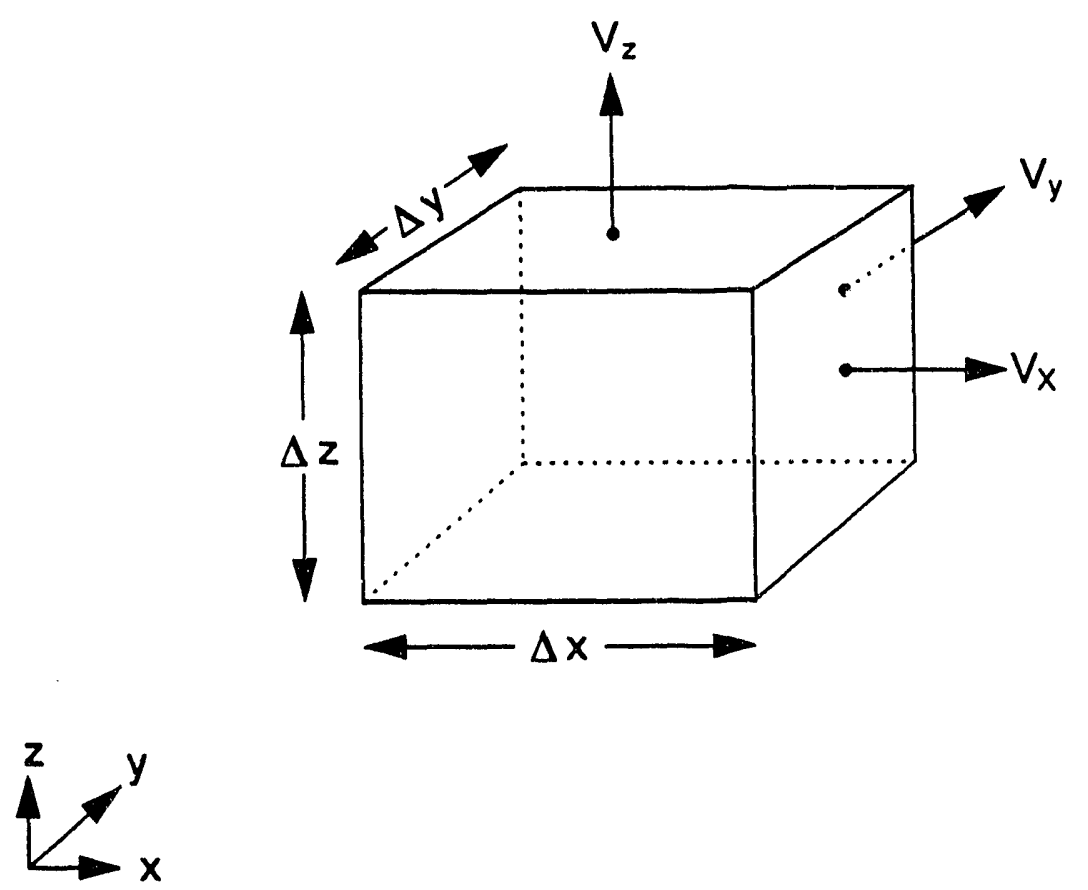

M552 rer-0492-03

Figure 2-3. Cartesian control volume. 
These are used in Equation (2-7), the discrete form of the mass equation, and Equation (2-11), the discrete form of the internal energy equation.

\subsubsection{Cylindrical Coordinates. For a finite cylindrical}

coordinate control volume as in Figure 2-4, the volume $V$ is given by

$$
\begin{aligned}
V=\int_{z_{1}}^{z_{2}} \int_{r_{1}}^{r_{2}} \int_{\theta_{1}}^{\theta_{2}} r d \theta d r d z & =\left.\frac{r^{2}}{2}\right|_{r_{1}} ^{r_{2}} \Delta \theta \Delta z=\frac{r_{2}^{2}-r_{1}^{2}}{2} \Delta \theta \Delta z \\
& =\left(r_{2}-r_{1}\right) \frac{\left(\frac{r_{2}+r_{1}}{2}\right) \Delta \theta \Delta z}{}
\end{aligned}
$$

or

$$
V=r_{m} \Delta r \Delta \theta \Delta z
$$

and for the area terms we have

$$
\begin{array}{ll}
r \text { - faces } & A_{r}=r_{m} \Delta \theta \Delta z \\
\theta \text { - faces } & A_{\theta}=\Delta r \Delta z \\
z \text { - faces } & A_{z}=\int_{\theta_{1}}^{\theta} \int_{1}^{r} r d r d \theta=r_{m} \Delta r \Delta \theta
\end{array}
$$

These are used in Equation (2-7), the discrete form of the mass equation, and Equation (2-11), the discrete form of the internal energy equation.

\subsection{Momentum Equations}

The momentum equations are used in their nonconservative form. In this way they can be used to advance the velocity components directly, instead of density times velocity. The partial differential equations in the 3 directions are of the form (Reference 2-1, p. 61)

$$
\rho\left(\frac{\partial \bar{v}}{\partial t}+\bar{v} \cdot \nabla \bar{v}\right)=-\nabla P+\bar{\sigma}+\rho \bar{f}
$$

For Cartesian coordinates, the momentum flux is given by 


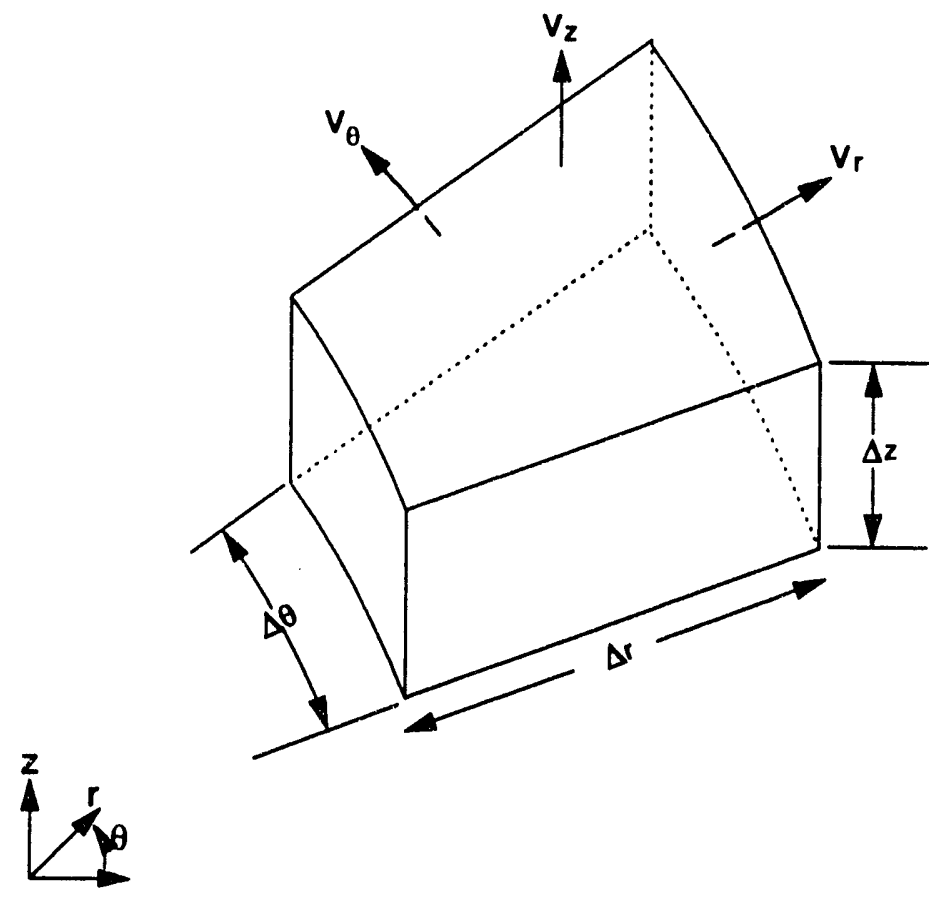

Ms52 rem-0nee-0use

Figure 2-4. Cylindrical control volume. 


$$
\bar{v} \cdot \nabla \bar{v}= \begin{cases}v_{x} \frac{\partial v_{x}}{\partial x}+v_{y} \frac{\partial v_{x}}{\partial y}+v_{z} \frac{\partial v_{x}}{\partial z} & \text { x component } \\ v_{x} \frac{\partial v_{y}}{\partial x}+v_{y} \frac{\partial v_{y}}{\partial y}+v_{z} \frac{\partial v_{y}}{\partial z} & \text { y component } \\ v_{x} \frac{\partial v_{z}}{\partial x}+v_{y} \frac{\partial v_{z}}{\partial y}+v_{z} \frac{\partial v_{z}}{\partial z} & z \text { component }\end{cases}
$$

and for cylindrical coordinates, the momentum flux is given by

$$
\bar{v} \cdot \nabla \bar{v}= \begin{cases}v_{r} \frac{\partial v_{r}}{\partial r}+\frac{v_{\theta}}{r} \frac{\partial v_{r}}{\partial \theta}-\frac{v_{\theta}^{2}}{r}+v_{z} \frac{\partial v_{r}}{\partial z} & r \text { component } \\ v_{r} \frac{\partial v_{\theta}}{\partial r}+\frac{v_{\theta}}{r} \frac{\partial v_{\theta}}{\partial \theta}+\frac{v_{r} v_{\theta}}{r}+v_{z} \frac{\partial v_{\theta}}{\partial z} & \theta \text { component } \\ v_{r} \frac{\partial v_{z}}{\partial r}+\frac{v_{\theta}}{r} \frac{\partial v_{z}}{\partial \theta}+v_{z} \frac{\partial v_{z}}{\partial z} & z \text { component }\end{cases}
$$

\subsubsection{Control Volume Forms}

To derive the control volume form of Equation (2-16), it is integrated over $v_{f}(t)$, using

$$
\int_{V_{f}(t)} \phi d V=\phi V_{f}=\alpha_{v} \phi V
$$

Here, $\Phi$ represents the average value of $\phi$ in the control volume. For ease of notation, the bar (-) over the $\phi$ will not be used in subsequent formulas. The notation for these terms can, at times, require many superscripts and subscripts (e.g., see Reference 2-2). The result is, dropping the superscript bar $(-)$,

$$
\alpha_{V} \rho V\left(\frac{\partial \bar{v}}{\partial t}+\bar{v} \cdot \nabla \bar{v}\right)=-\alpha_{V} V \nabla P+\int_{V_{f}(t)} \bar{\sigma} d V+\alpha_{v} \rho \bar{f} V
$$




\subsubsection{Discrete Forms with Approximations}

The control volumes for momentum are staggered as in the ICE scheme. 2-3 Other guidelines are as follows:

1. The time derivatives and convective terms are discretized with old $n$-time levels on the coefficients.

2. All derivative terms in the convective terms are donored, i.e., the gradients are calculated using upstream values for the finite difference divided differences. 01d $n$-time levels are used for all terms in the convective terms.

3. $\bar{\sigma}$ represents the viscous stress gradient terms in Reference 2-1, p. 61. This term will give rise (after use of the Divergence Theorem) to three terms for (a) viscous stress acting on the surface $S_{C}$ occupied by fluid; these viscous stress are generally small; (b) viscous stress acting on the part of $S_{c}$ that consists of solid wall; this gives the normal wall drag term, and $(c)$ viscous stresses acting on $S_{j}(t)$ and these give the normal interface drag terms.

4. The wall and interface drag correlations must satisfy certain invariant requirements that follow from the symmetry of the imbedded solid structure. These forms are implicit in the velocities.

2.2.2.1 Cartesian Coordinates. For volume V, Equation (2-12) is used. For lengths, $\Delta x, \Delta y$, and $\Delta z$ are used. The momentum flux is given by

$$
\bar{v} \cdot \nabla \bar{v}=\left\{\begin{array}{cc}
v_{x} \frac{\Delta v_{x}}{\Delta x}+v_{y} \frac{\Delta v_{x}}{\Delta y}+v_{z} \frac{\Delta v_{x}}{\Delta z} & \text { x component } \\
v_{x} \frac{\Delta v_{y}}{\Delta x}+v_{y} \frac{\Delta v_{y}}{\Delta y}+v_{z} \frac{\Delta v_{y}}{\Delta z} & \text { y component } \\
v_{x} \frac{\Delta v_{z}}{\Delta x}+v_{y} \frac{\Delta v_{z}}{\Delta y}+v_{z} \frac{\Delta v_{z}}{\Delta z} & z \text { component }
\end{array}\right.
$$


2.2.2.2 Cylindrical Coordinates. For volume V, Equation (2-14) is used. For lengths, $\Delta r, r_{m} \Delta \theta$, and $\Delta z$ are used. The momentum flux is given by

$$
\bar{v} \cdot \nabla \bar{v}= \begin{cases}v_{r} \frac{\Delta v_{r}}{\Delta r}+\frac{v_{\theta}}{r_{m}} \frac{\Delta v_{r}}{\Delta \theta}-\frac{v_{\theta}^{2}}{r_{m}}+v_{z} \frac{\Delta v_{r}}{\Delta z} & r \text { component } \\ v_{r} \frac{\Delta v_{\theta}}{\Delta r}+\frac{v_{\theta}}{r_{m}} \frac{\Delta v_{\theta}}{\Delta \theta}+\frac{v_{r} v_{\theta}}{r_{m}}+v_{z} \frac{\Delta v_{\theta}}{\Delta z} & \theta \text { component } \\ v_{r} \frac{\Delta v_{z}}{\Delta r}+\frac{v_{\theta}}{r_{m}} \frac{\Delta v_{z}}{\Delta \theta}+v_{z} \frac{\Delta v_{z}}{\Delta z} & z \text { component }\end{cases}
$$

\subsubsection{Shallow Water Equations}

Flow at low velocity can be stratified as a result of buoyancy forces caused by density differences between vapor and liquid. When the flow is stratified, the area average pressures are affected by nonuniform transverse distribution of the phases. As a result, an extra force term is needed in the momentum equation. This term is already present in RELAP5 for 1-D components, ${ }^{2-4}$ and this section shows its derivation for 3-D components. The term is particularly important for shallow water situations where the flow is stratified.

The hydrostatic forces (pressure) assuming small vertical motion are shown in Figure 2-5, with $P_{T}, P_{I}$, and $P_{B}$ related by:

$$
\begin{aligned}
& P_{I}=P_{T}+\alpha_{g} \rho_{g} h g, \\
& P_{B}=P_{I}+\alpha_{f} \rho_{f} h g .
\end{aligned}
$$

The force on the gas due to this pressure distribution is shown in Figure 2-6, and is given by

$$
-\left(h \alpha_{g} \bar{P}_{g}\right)_{x+\Delta x}+\underbrace{\left(h \alpha_{g} \bar{P}_{g}\right)_{x}}_{\text {forces on ends }}+\underbrace{P_{I}\left(\left.\alpha_{g} h\right|_{x+\Delta x}-\left.\alpha_{g} h\right|_{x}\right)}_{\begin{array}{l}
\text { axial force due to } \\
\text { sloping interface }
\end{array}}
$$




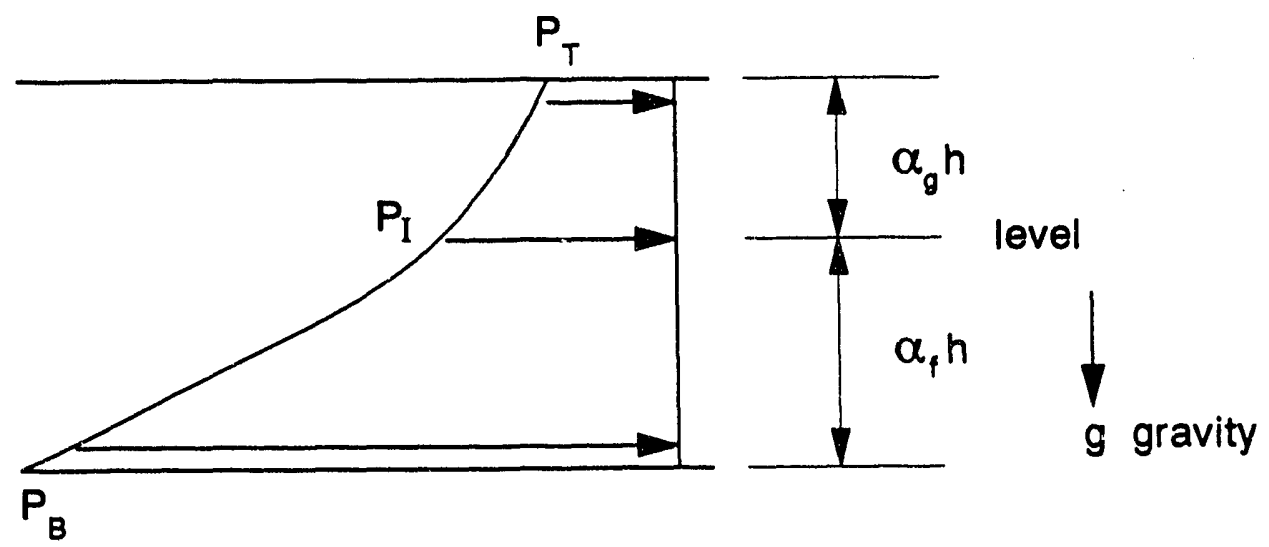

Figure 2-5. Hydrostatic forces (pressure). 


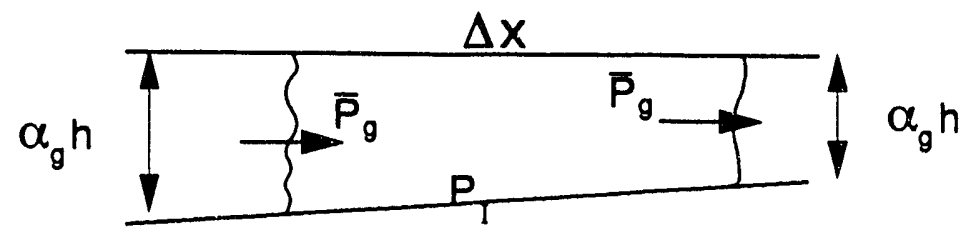

M552 rar-0492-06

Figure 2-6. Force on gas due to pressure distribution. 
where $\tilde{P}_{g}$ is the average pressure at a face in the gas, i.e.,

$$
\bar{P}_{g}=\frac{\left(P_{T}+P_{I}\right)}{2}
$$

Taking the limit in Equation (2-26) per unit length gives (also divided by $h$ )

$$
\begin{aligned}
\text { force on gas } & =-\frac{\partial\left(\alpha_{g} \bar{P}_{g}\right)}{\partial x}+P_{I} \frac{\partial \alpha_{g}}{\partial x} \\
& =-\alpha_{g} \frac{\partial \bar{P}_{g}}{\partial x}+\left(P_{I}-\bar{P}_{g}\right) \frac{\partial \alpha_{g}}{\partial x}
\end{aligned}
$$

Now using Equations (2-2s) and (2-23)

$$
\bar{P}_{g}=\frac{\left(P_{I}-\alpha_{g} \rho_{g} h g\right)+P_{I}}{2}=P_{I}-\frac{\alpha_{g} \rho_{g} h g}{2}
$$

and substituting this into Equation (2-27) for $\overline{\mathrm{p}}_{\mathrm{g}}$ gives

$$
\text { force on gas }=-\alpha_{g}\left(\frac{\partial P_{I}}{\partial x}-\frac{\rho_{q}{ }^{h g}}{2} \frac{\partial \alpha_{g}}{\partial x}\right)+\frac{\alpha_{q} \rho_{g} h g}{2} \frac{\partial \alpha_{g}}{\partial x}
$$

or

$$
\text { force on gas }=-\alpha_{g} \frac{\partial P_{I}}{\partial x}+\alpha_{g} \rho_{g} h g \frac{\partial \alpha_{g}}{\partial x}
$$

A parallel development for the force on the liquid leads to

$$
\text { force on liquid }=-\alpha_{f} \frac{\partial \tilde{P}_{f}}{\partial x}+\left(P_{I}-\tilde{P}_{f}\right) \frac{\partial \alpha_{f}}{\partial x}
$$

where

$$
\tilde{P}_{f}=\frac{P_{I}+P_{B}}{2}
$$

As before this reduces to

$$
\text { force on liquid }=-\alpha_{f} \frac{\partial P_{I}}{\partial x}+\alpha_{f} \rho_{f} h g \frac{\partial \alpha_{g}}{\partial x}
$$


We now define how $P_{I}$ (or $P_{T}$ or $P_{B}$ ) is related to the mean pressure $P$ calculated by the code.

The code will make the pressure calculation based upon a vertical force balance assuming a mixed flow as shown in Figure 2-7 with a gravity head relating $P_{T}$ and $P_{B}$. We note that adding the vertical momentum equation keeping only pressure and gravity terms gives

$$
0=-\frac{\partial P}{\partial z}+\alpha_{g} \rho_{g} g+\alpha_{f} \rho_{f} g
$$

and integrating over the control volume in the $z$-direction gives

$$
P_{B}=P_{T}+\alpha_{g} \rho_{g} g h+\alpha_{f} \rho_{f} g h
$$

which gives the same $P_{B}-P_{T}$ as given by Equations (2-23) and (2-24), i.e., stratified or not we get the same total $\Delta P$ in the $z$-direction, it is just distributed differently in the z-direction.

If we use the present RELAP5 convention for mean pressure [Equation (53), Reference 2-4] i.e.,

$$
p \triangleq \alpha_{g} \bar{P}_{g}+\alpha_{f} \bar{P}_{f}
$$

then we get

$$
P=\alpha_{g}\left(\frac{P_{T}+P_{I}}{2}\right)+\alpha_{f}\left(\frac{P_{I}+P_{B}}{2}\right)
$$

and using Equations (2-23) and (2-24), this becomes

$$
P=P_{I}-\frac{\alpha_{g}{ }^{2} \rho_{q} h g}{2}+\frac{\alpha_{f}{ }^{2} \rho_{f} h g}{2}
$$

Putting this into Equation $(2-28)$ gives 


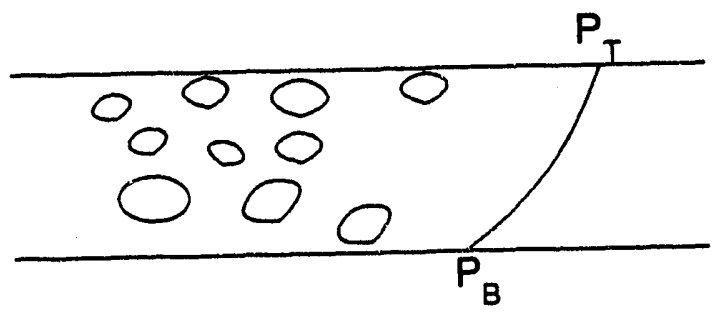

M552 rar-0492-07

Figure 2-7. Mixed flow pressure distribution. 


$$
\begin{aligned}
& \text { force on gas }=-\alpha_{g}\left[\frac{\partial P}{\partial x}+\frac{\partial}{\partial x}\left(\frac{\alpha_{g}^{2} \rho_{g} h g}{2}\right)-\frac{\partial}{\partial x}\left(\frac{\alpha_{f}^{2} \rho_{f} h g}{2}\right)\right] \\
& +\alpha_{g} \rho_{g} h g \frac{\partial \alpha_{g}}{\partial x} \\
& =-\alpha_{g} \frac{\partial P}{\partial x}+\alpha_{f} \alpha_{g}\left(\rho_{g}-\rho_{f}\right) h g \frac{\partial \alpha_{g}}{\partial x}
\end{aligned}
$$

Putting Equation (2-34) into Equation (2-29) gives

$$
\begin{aligned}
& \text { force on liquid }=-\alpha_{f}\left[\frac{\partial P}{\partial x}+\frac{\partial}{\partial x}\left(\frac{\alpha_{g}^{2} \rho_{g} h g}{2}\right)-\frac{\partial}{\partial x}\left(\frac{\alpha_{f}^{2} \rho_{f} h g}{2}\right)\right] \\
& +\alpha_{f} \rho_{f} h g \frac{\partial \alpha_{g}}{\partial x} \\
& =-\alpha_{f} \frac{\partial P}{\partial x}+\alpha_{f} \alpha_{g}\left(\rho_{f}-\rho_{g}\right) h g \frac{\partial \alpha_{g}}{\partial x}
\end{aligned}
$$

For Cartesian coordinates, Equations (2-35) and (2-36) show what $\alpha \frac{\partial P}{\partial x}$ and $\alpha \frac{\partial P}{\partial y}$ in Equation $(2-20)$ are replaced by to make stratified forces in the horizontal region. For cylindrical coordinates, Equations (2-35) and (2-36) show what $\alpha \frac{\partial \mathrm{P}}{\partial r}$ and $\alpha \frac{\partial \mathrm{P}}{\partial \theta} \frac{1}{r}$ in Equation $(2-20)$ are replaced by to make stratified forces in the horizontal region.

\subsection{References}

2-1. J. C. Slattery, Momentum, Energy, and Mass Transfer in Continua, 2nd Edition, McGraw-Hill, New York, 1979.

2-2. V. H. Ransom, Course A - Numerical Modeling of Two-Phase Flows for Presentation at Ecole d'Ete d'Analyse Numerique, EGG-EAST-8546, May 1989.

2-3. F. Harlow and A. Amsden, Numerical Calculation of Almost Incompressible Flow, Journal of Computational Physics, 3,1969 , pp. 80-93.

2-4. V. H. Ransom et al., RELAP5/MOD2 Code Marual, Volume 1 , NUREG/CR-4312, EGG-2396, Revision 1, March 1987. 


\subsection{IMPLEMENTATION OF THE}

\section{MULTIDIMENSIONAL MODEL TWO-PHASE FLOW EQUATIONS}

\subsection{INTRODUCTION}

This section presents the implementation of the momentum flux terms included in the momentum equations and how they are evaluated in the interior, as well as at the boundaries of a 3-D region. Additional considerations for 1-D to 3-D connections are also given. Lastly, details of code internals which directly affect the new model are presented. The modifications to the mass and energy equations are minor (e.g., areas and volume), and they are discussed in the previous Section 2.

This discussion of the 3-D model will first be given in terms of the Cartesian coordinate system for clarity. Later, sections will present the equations in cylindrical coordinates and their implementation.

\subsection{Cartesian Coordinates}

For the convective terms, we are interested only in the spatial gradient terms from Equation (2-16). The Cartesian form of the spatial gradient terms of the momentum flux terms are given in Equation (2-17), and are repeated here for reference. Tney are:

$$
\begin{aligned}
& v_{x} \frac{\partial v_{x}}{\partial x}+v_{y} \frac{\partial v_{x}}{\partial y}+v_{z} \frac{\partial v_{x}}{\partial z}, \\
& v_{x} \frac{\partial v_{y}}{\partial x}+v_{y} \frac{\partial v_{y}}{\partial y}+v_{z} \frac{\partial v_{y}}{\partial z} \text {, and } \\
& v_{x} \frac{\partial v_{z}}{\partial x}+v_{y} \frac{\partial v_{z}}{\partial y}+v_{z} \frac{\partial v_{z}}{\partial z}
\end{aligned}
$$

The finite difference form and implementation of these equations is given in the next section. 


\subsubsection{Momentum Flux Terms}

The momentum flux terms in finite difference form will be presented here. Considerations for boundary conditions and external connections will be given in the following sections. Although these difference equations are straight forward, the interior cell equations will be discussed before doing the side cell or corner cell forms. The index notation is shown in Figure 3-1 for the ' $x-y$ ' plane, and Figure 3-2 for the ' $x-z$ ' plane. The variables ' $v_{x}$ ', ' $v_{y}$ ', and ' $v_{z}$ ' are the $x, y$, and $z$ velocities respectively, the subscripts indicate the cell edges where the velocities are defined.

This is done on the control volume for the momentum cell about $v_{x_{i+1 / 2, j, k}}$ (Figure 3-3). Using a donor formulation where the spatial gradient terms are evaluated on the upstream side relative to the velocity that multiples them, the convective terms become in finite difference form

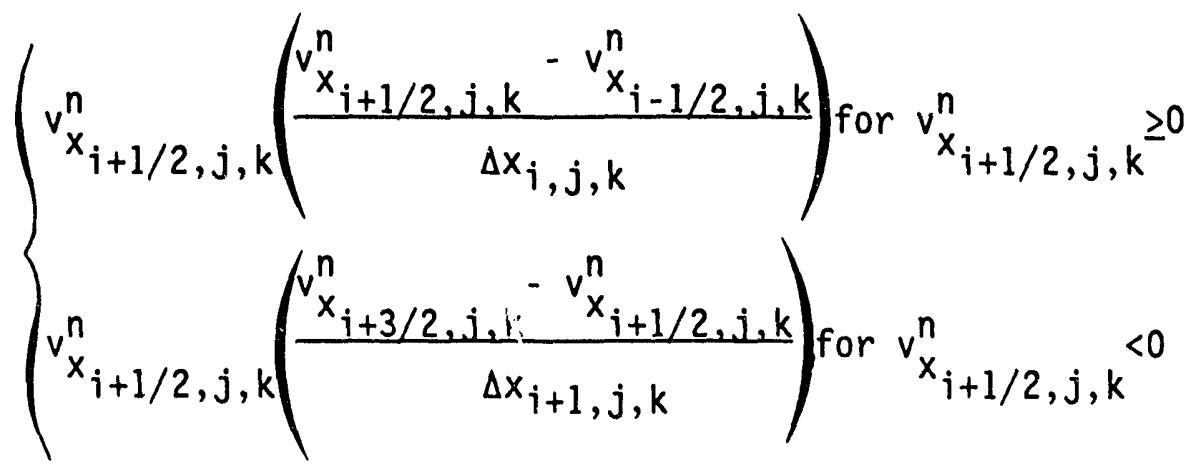

$$
\begin{aligned}
& +\left(\begin{array}{l}
\bar{v}_{y_{i+1 / 2, j, k}^{n}}\left(\frac{v_{i+1 / 2, j, k}^{n}-v_{x_{i+1 / 2, j-1, k}}^{n}}{\frac{1}{2}\left(\Delta y_{i, j, k}+\Delta y_{i, j-1, k}\right)}\right) \text { for } v_{y_{i+1 / 2, j, k}^{n} \geq 0}^{n}\left(\frac{v_{x_{i+1 / 2, j+1, k}}^{n}-v_{x_{i+1 / 2, j, k}}^{n}}{\frac{1}{2}\left(\Delta y_{i, j+1, k}+\Delta y_{i, j, k}\right)}\right) \text { for } v_{y_{i+1 / 2, j, k}^{n}}^{n}<0
\end{array}\right.
\end{aligned}
$$




$$
\left\{\begin{array}{l}
v_{z_{i+1 / 2, j, k}^{n}}^{n}\left(\frac{v_{x_{i+1 / 2, j, k}}^{n}-v_{x_{i+1 / 2, j, k-1}^{n}}^{n}}{\frac{1}{2}\left(\Delta z_{i, j, k}+\Delta z_{i, j, k-1}\right)}\right) \text { for } v_{z_{i+1 / 2, j, k}}^{n} \geq 0 \\
\bar{v}_{z_{i+1 / 2, j, k}}^{n}\left(\frac{v_{x_{i+1 / 2, j, k+1}^{n}}^{n}-v_{x_{i+1 / 2, j, k}}^{n}}{\frac{1}{2}\left(\Delta z_{i, j, k+1}+\Delta z_{i, j, k}\right)}\right) \text { for } \bar{v}_{z_{i+1 / 2, j, k}}^{n}<0
\end{array}\right.
$$

In Equation (3-2), the velocities not located on the junction plane $' i+1 / 2$ ', i.e., $\bar{v}_{y_{i+1 / 2, j, k}}$ are the direct average of the four surrounding junction velocities. Namely,

$$
\bar{v}_{y_{i+1 / 2, j, k}}^{n}=\frac{1}{4}\left(v_{y_{i, j-1 / 2, k}}^{n}+v_{y_{i, j+1 / 2, k}}^{n}+v_{y_{i+1, j-1 / 2, k}}^{n}+v_{y_{i+1, j+1 / 2, k}}^{n}\right)
$$

and

$$
v_{z_{i+1 / 2, j, k}}^{n}=\frac{1}{4}\left(v_{z_{i, j, k-1 / 2}}^{n}+v_{z_{i, j, k+1 / 2}^{n}}^{n}+v_{z_{i+1, j, k-1 / 2}^{n}}^{n}+v_{z_{i+1, j, k+1 / 2}^{n}}^{n}\right)
$$

The finite difference approximation for the $y$-direction convective term in the momentum cell about $v_{y_{i, j+1 / 2, k}}$, (Figure 3-4), becomes

$$
\left\{\begin{array}{l}
v_{y_{i, j+1 / 2, k}}^{n}\left(\frac{v_{y_{i, j+1 / 2, k}}^{n}-v_{y_{i, j-1 / 2, k}}^{n}}{\Delta y_{i, j, k}}\right) \text { for } v_{y_{i, j+1 / 2, k}}^{n} \geq 0 \\
v_{y_{i, j+1 / 2, k}}^{n}\left(\frac{v_{y_{i, j+3 / 2, k}}^{n}-v_{y_{i, j+1 / 2, k}}^{n}}{\Delta y_{i, j+1, k}}\right) \text { for } v_{y_{i, j+1 / 2, k}}^{n}<0
\end{array}\right.
$$




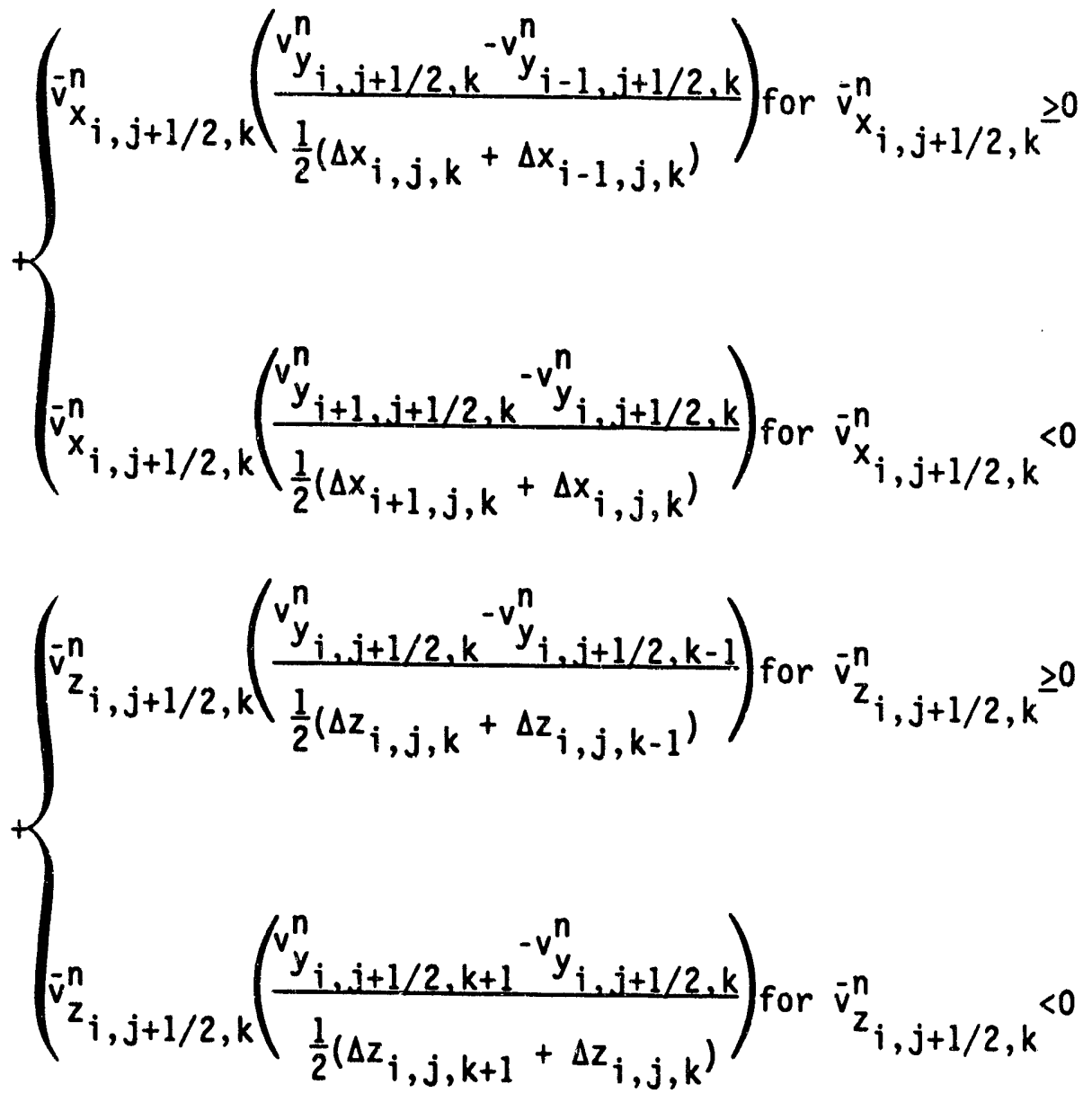

Again, the junction velocities that are not on the ' $j+1 / 2$ ' $p l$ ane are given by

$$
v_{x_{i, j+1 / 2, k}}^{n}=\frac{1}{4}\left(v_{x_{i-1 / 2, j, k}^{n}}^{n}+v_{x_{i+1 / 2, j, k}^{n}}^{n}+v_{x_{i-1 / 2, j+1, k}^{n}}^{n}+v_{x_{i+1 / 2, j+1, k}}^{n}\right)
$$

and

$$
v_{z_{i, j+1 / 2, k}}^{-n}=\frac{1}{4}\left(v_{z_{i, j, k-1 / 2}^{n}}+v_{z_{i, j, k+1 / 2}^{n}}+v_{z_{i, j+1, k-1 / 2}^{n}}+v_{z_{i, j+1, k+1 / 2}^{n}}\right)
$$

Finally, the convective term for the $z$-face, in finite difference form over the $z$ control volume, (Figure 3-5) are 


$$
\begin{aligned}
& \left\{\begin{array}{l}
v_{z_{i, j, k+1 / 2}^{n}}\left(\frac{v_{z_{i, j, k+1 / 2}^{n}}-v_{z_{i, j, k-1 / 2}^{n}}^{n}}{\Delta z_{i, j, k}}\right) \text { for } v_{z_{i, j, k+1 / 2}^{n} \geq 0} \\
v_{z_{i, j, k+1 / 2}^{n}}^{n}\left(\frac{v_{z_{i, j, k+3 / 2}}^{n}-v_{z_{i, j, k+1 / 2}}^{n}}{\Delta z_{i, j, k+1}}\right) \text { for } v_{z_{i, j, k+1 / 2}^{n}}<0
\end{array}\right. \\
& \left\{\begin{array}{l}
\sum_{x_{i, j, k+1 / 2}}^{n}\left(\frac{v_{z_{i, j, k+1 / 2}}^{n}-v_{z_{i-1, j, k+1 / 2}}^{n}}{\frac{1}{2}\left(\Delta x_{i, j, k}+\Delta x_{i-1, j, k}\right)}\right) \text { for } v_{x_{i, j, k+1 / 2}}^{n} \geq 0 \\
i_{x_{i, j, k+1 / 2}}^{n}\left(\frac{v_{z_{i+1, j, k+1 / 2}^{n}}^{n}-v_{z_{i, j, k+1 / 2}}^{n}}{\frac{1}{2}\left(\Delta x_{i+1, j, k}+\Delta x_{i, j, k}\right)}\right) \text { for } v_{x_{i, j, k+1 / 2}^{n}}^{n}<0
\end{array}\right.
\end{aligned}
$$

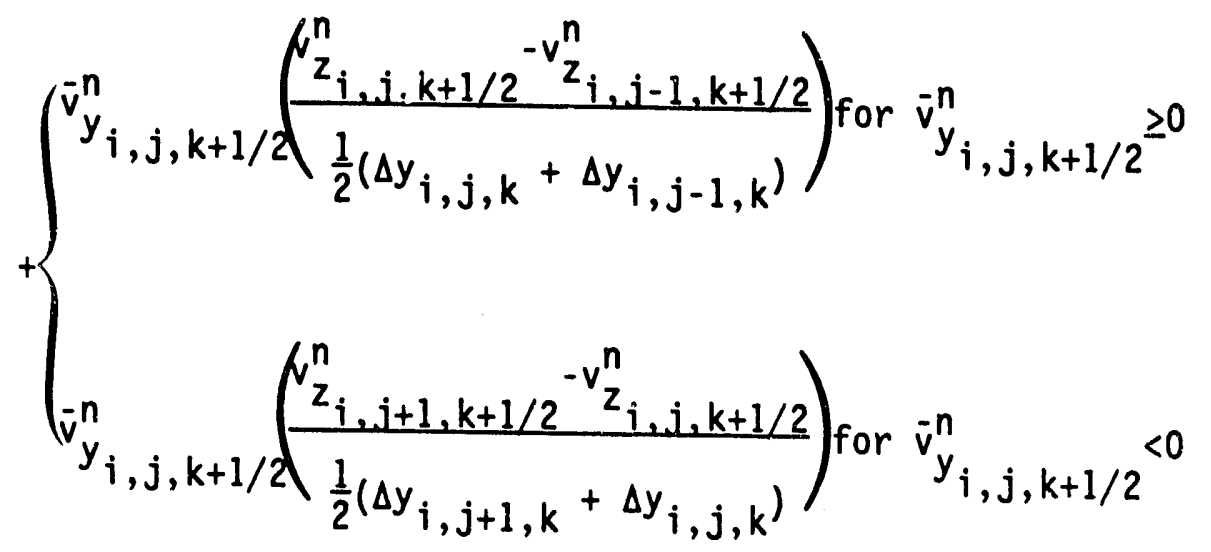

where the overscore terms are the averaged velocities

$$
v_{x_{i, j, k+1 / 2}}^{n}=\frac{1}{4}\left(v_{x_{i-1 / 2, j, k}}^{n}+v_{x_{i+1 / 2, j, k}}^{n}+v_{x_{i-1 / 2, j, k+1}}^{n}+v_{x_{i+1 / 2, j, k+1}}^{n}\right)
$$

and

$$
\bar{v}_{y_{i, j, k+1 / 2}}^{n}=\frac{1}{4}\left(v_{y_{i, j-1 / 2, k}}^{n}+v_{y_{i, j+1 / 2, k}}^{n}+v_{y_{i, j-1 / 2, k+1}^{n}}^{n}+v_{y_{i, j+1 / 2, k+1}}^{n}\right)
$$

3-5 


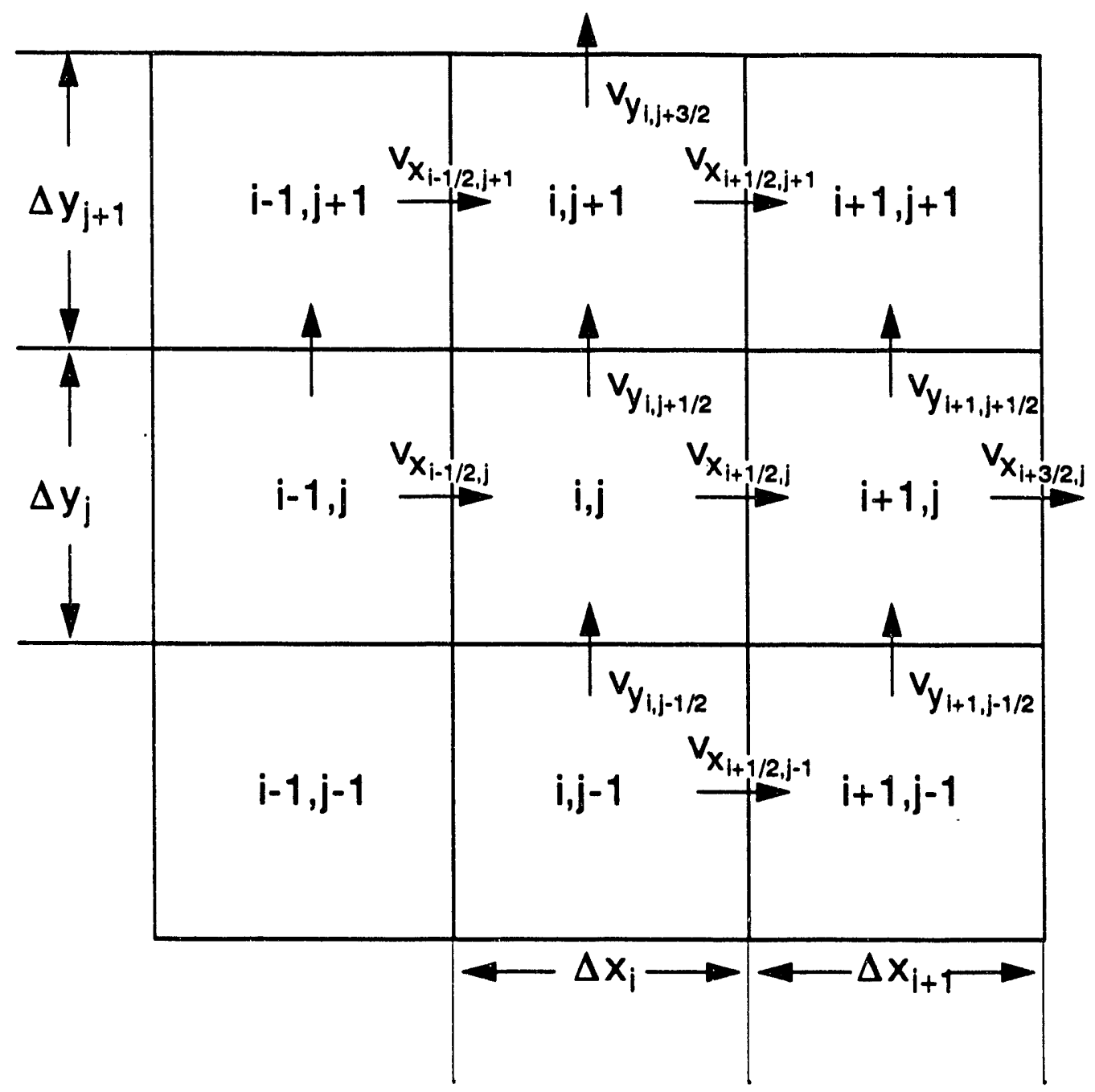

MSEe rer-04se-0s

Figure 3-1. Interior cells for $x y \mathrm{plane}$. 


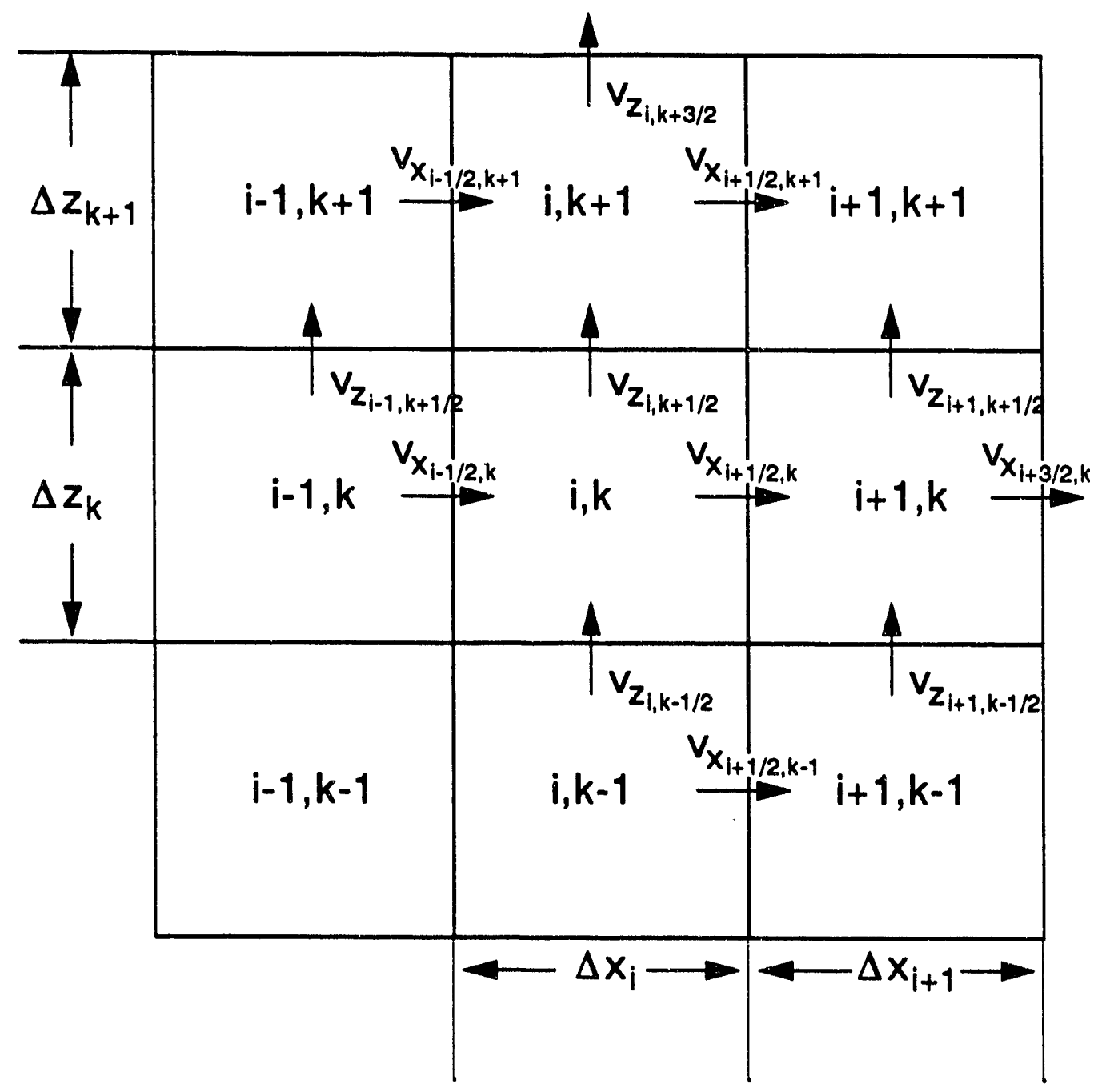

MS5e ra-0482-00

Figure 3-2. Interior cells for $x z$ plane. 


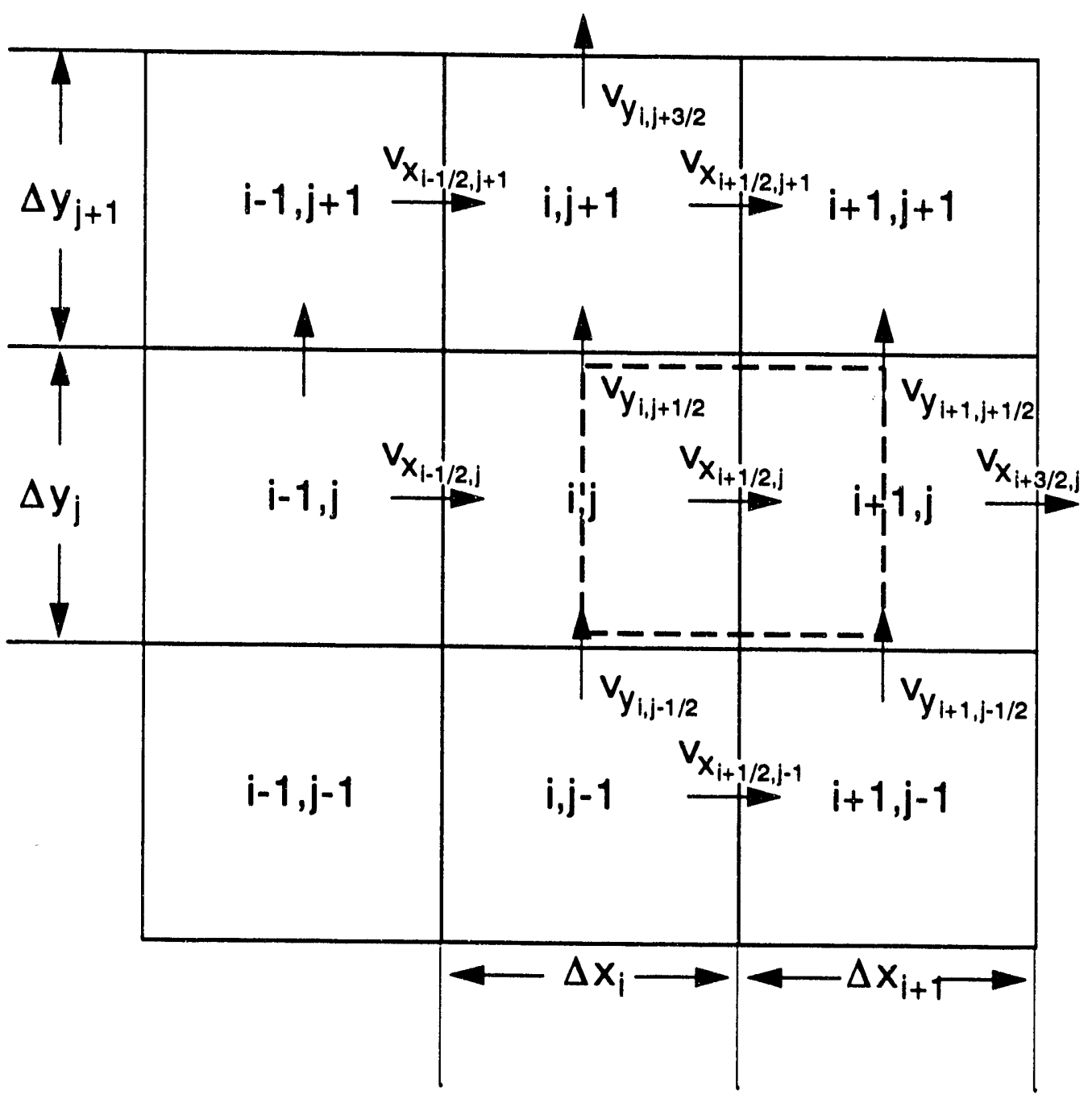

M552 rer.0492-10

Figure 3-3. Control volume for the momentum cell about $v_{x_{i+1 / 2, j, k}}$. 


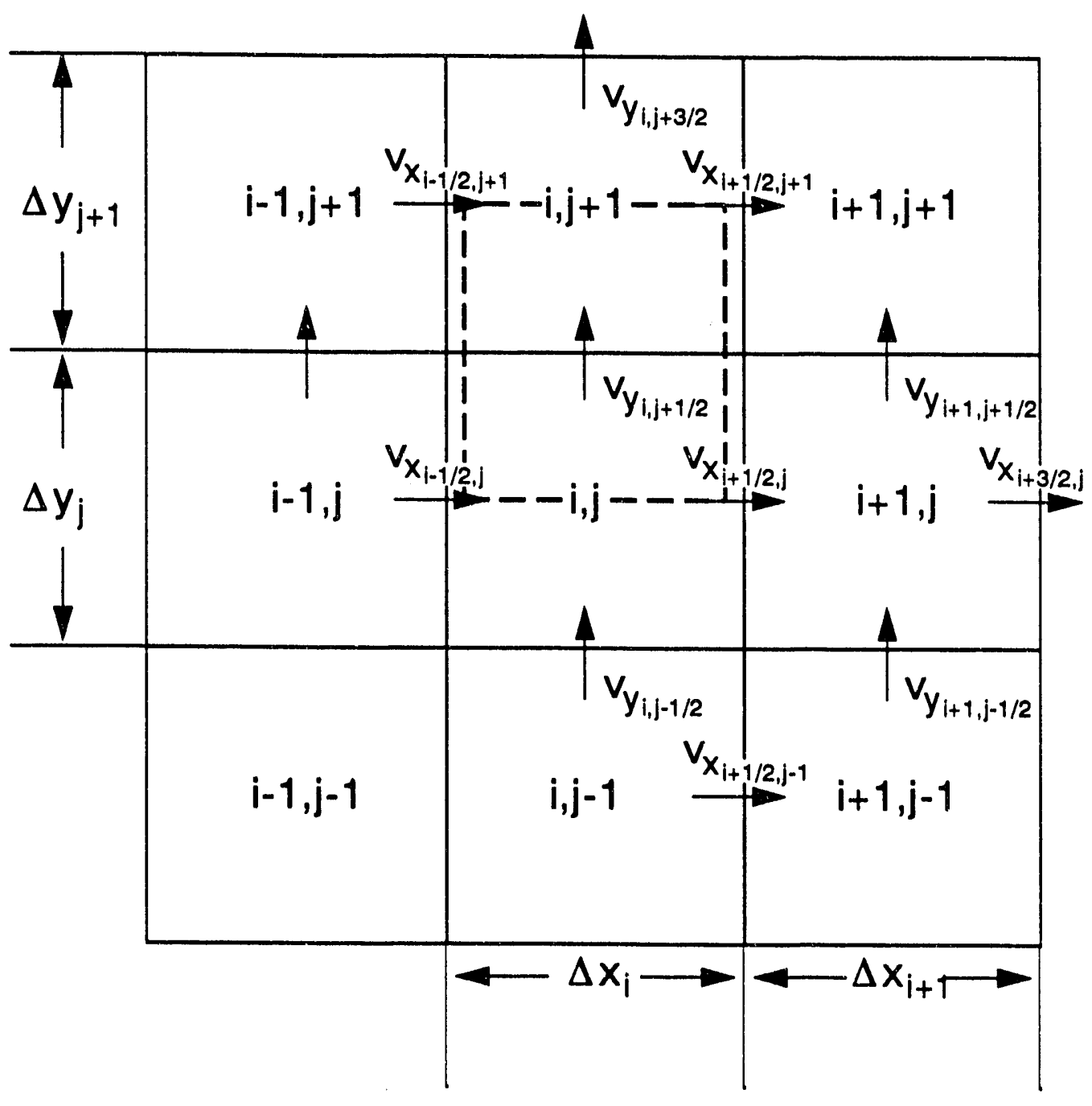

M552 rer-0492-11

Figure 3-4. Control volume for the momentum cell about $v_{y_{i, j+1 / 2, k}}$. 


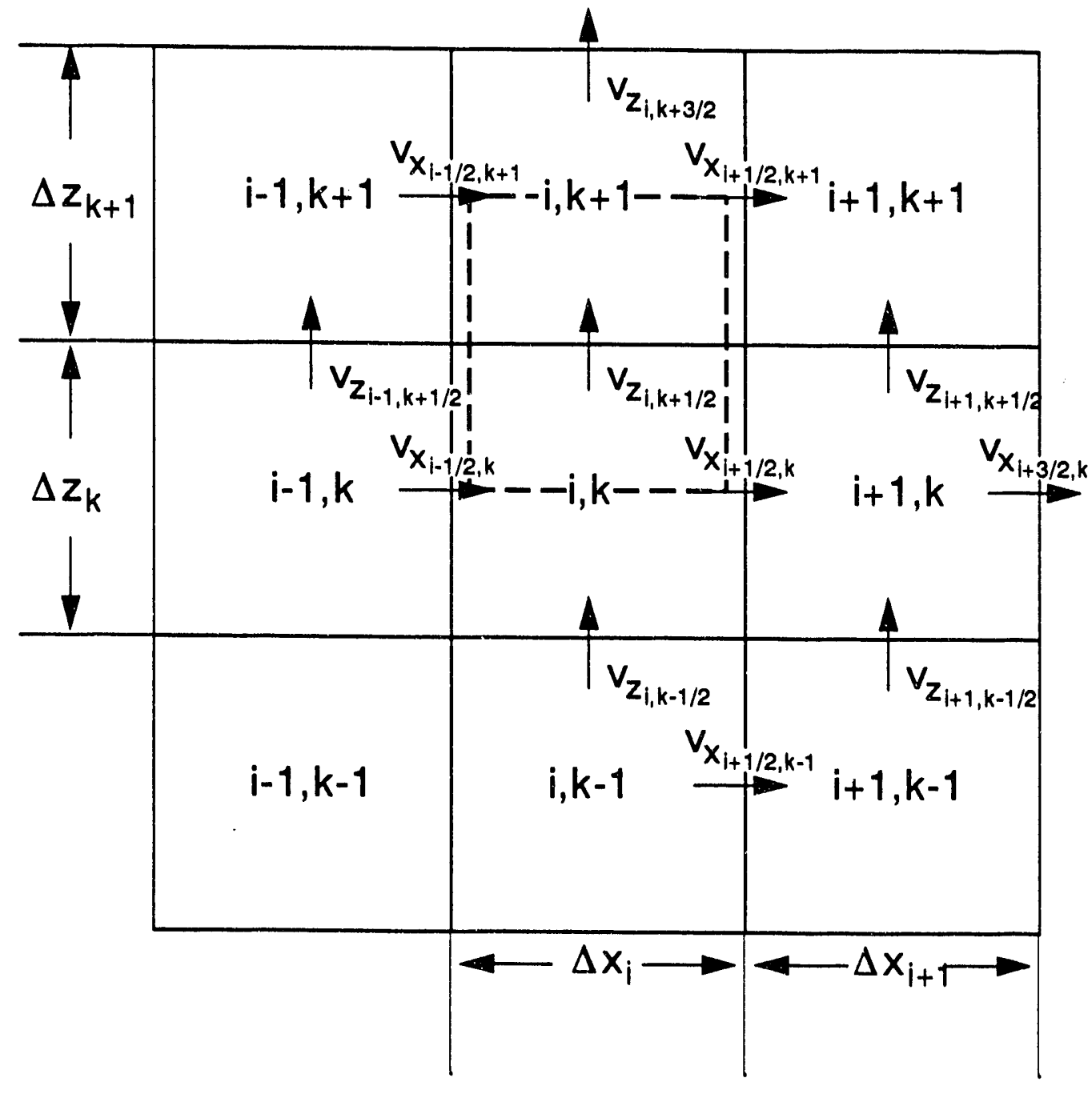

M552 rer-0492-12

Figure 3-5. Control volume for the momentum cell about $v_{z_{i, j, k+1 / 2}}$. 


\subsubsection{Terms at Interior Boundaries}

The terms for the momentum equations tangent and normal to an interior boundary require special treatment. Free slip at these boundaries is assumed. This is the correct boundary condition when there are no viscous terms coded, since the fluid is modeled as an inviscid fluid with internal resistance modeled using friction factors. The mathematical boundary conditions are (a) normal velocity (normal to wall) is zero, and (b) the shear stress on the wall is zero.

Now the shear stress is given by

$$
\sigma_{x y}=\mu\left(\frac{\partial v_{x}}{\partial y}+\frac{\partial v_{y}}{\partial x}\right)
$$

where $\mu$ is the viscosity. From condition (a) $\left(v_{x}=0\right.$ on wall), we have $\partial v_{x} / \partial y=0$ at the wall and from the second condition, $\frac{\partial v_{y}}{\partial x}=0$ at the wall for $\sigma_{x y}=0$. Hence the two velocity conditions are

$$
\text { (a) }\left.v_{x}\right|_{\text {wall }}=0
$$

and

(b) $\left.\frac{\partial v_{y}}{\partial x}\right|_{\text {wa11 }}=0$

In the code, provisions are made to store these boundary velocities. The 3-D component is surrounded by "pseudo-cells" which contain the same type of information as the interior cells, but these cells are not included in the momentum flux calculation. They provide a convenient method for setting the boundary velocities.

3.2.2.1 Side Cells. Discussions of the ' $x-y$ plane' case is given since the extension to the full 3-dimensional case is straightforward. Figure 3-6 shows the same cells as Figure $3-1$, but located next to a side wall. This figure shows all the usual junction velocities that are defined with the multidimensional component with solid arrows and all the other junction velocities needed for the momentum equations as shaded arrows. These other velocities are a function of the boundary conditions at the wall. 


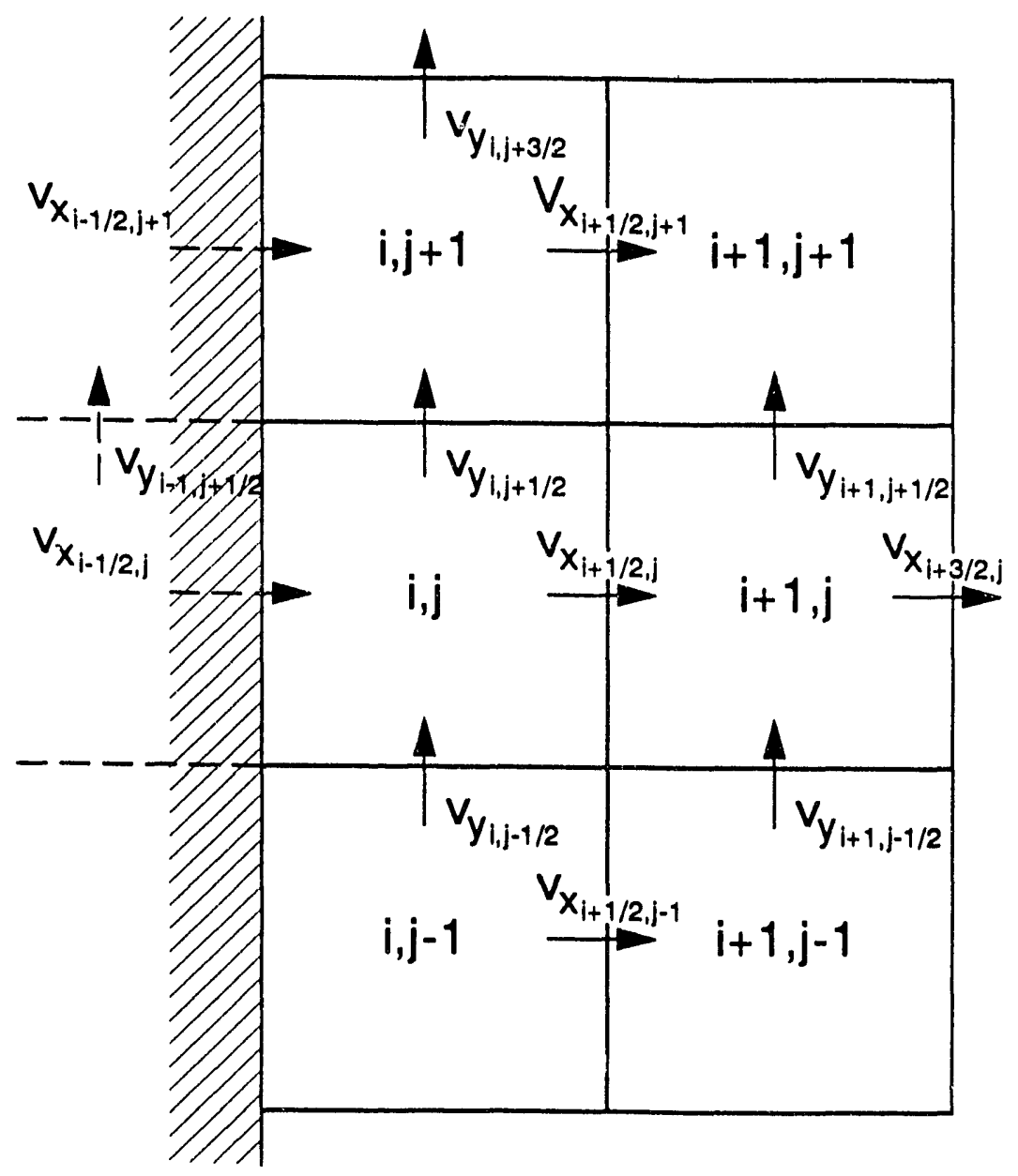

M552 rex-0482-13

Figure 3-6. Side cell notation. 
For these cells, the finite difference equations for $v_{x_{i+1 / 2, j}}$ and $v_{y_{i, j+1 / 2}}$ need to be discussed. In writing the interior cell equations for $v_{x_{i+1 / 2, j}}$ and $v_{y_{i, j+1 / 2}}$, the usual junction velocities plus the velocities indicated by shaded arrows that do not exist in the multidimensional component would be needed. Thus velocities normal to the momentum cell velocity are set to zero and velocities tangent to the momentum cell velocity are set to that momentum cell velocity, and are given by

$$
\begin{aligned}
& v_{x_{i-1 / 2, j}}^{n}=0 \\
& v_{x_{i-1 / 2, j+1}^{n}}^{n}=0
\end{aligned}
$$

and

$$
v_{y_{i-1, j+1 / 2}}^{n}=v_{y_{i, j+1 / 2}}^{n}
$$

If these 'boundary condition' values given by Equations (3-14) and (3-15) are used for the shaded velocities in Figure 3-6, then Equations (3-2) and $(3-5)$, will hold at a side cell.

\subsubsection{Corner Cells. Additional considerations must be made at} corners. Figure 3-7 shows all the junctions velocities that can be obtained from interior cell equations ( $i$ superscript) or side cell equations (s superscripts). The single $v_{x}^{c}$ or $v_{y}^{c}$ (superscript $c$ meaning corner cell junction velocities)must be determined using corner cell momentum equations.

Figure 3-8, shows a corner where the corner cell $v_{x}^{c}$ velocity is $v_{x_{i+1 / 2, j}}$. All the velocities needed to evaluate the $x$-momentum equation for $v_{x_{i+1 / 2, j}}$ at an interior cell are shown. The solid arrows are normal junction velocities from the multidimensional component, and the shaded arrows represent the velocities that must be obtained by extrapolated boundary conditions: 


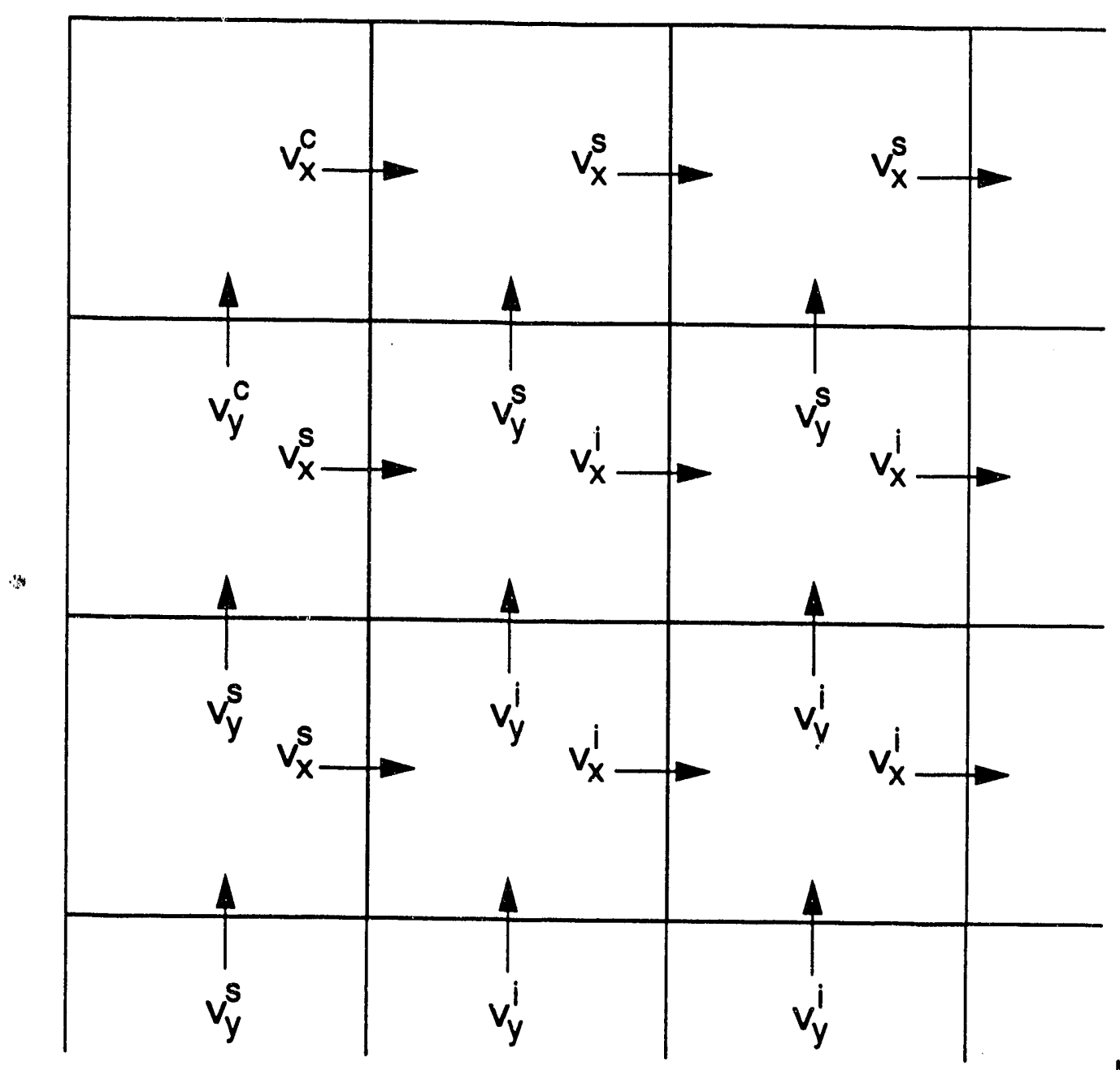

M552 rer-0482-14

Figure 3-7. Interior, side, and corner cells. 


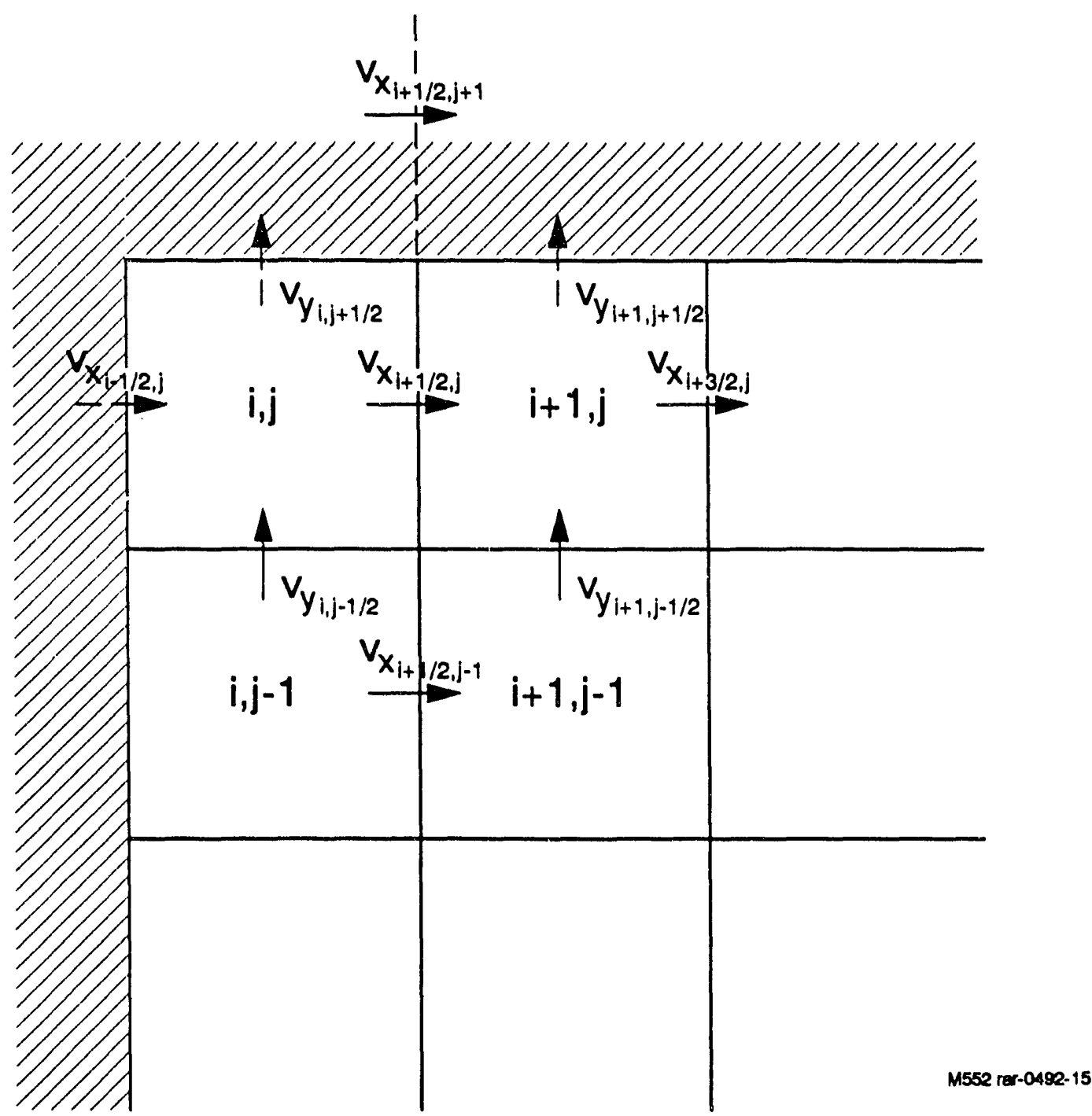

Figure 3-8. Corner cell extrapolation velocities for x-momentum equation. 


$$
\begin{aligned}
& v_{x_{i-1 / 2, j}}^{n}=0 \\
& v_{x_{i+1 / 2, j+1}^{n}}^{n}=v_{x_{i+1 / 2, j}}^{n} \\
& v_{y_{i, j+1 / 2}^{n}}^{n}=0 \\
& v_{y_{i+1, j+1 / 2}^{n}}^{n}=0
\end{aligned}
$$

Figure 3-9, shows a corner/side region where the velocity must be set by extrapolation. All the velocities needed to evaluate the y-momentum equation for $v_{y}, j+1 / 2$ are shown. As before, the solid arrows are the defined velocities from the multidimensional component, and the velocities next to the shaded arrows come from the extrapolated boundary conditions:

$$
\begin{aligned}
& v_{x_{i-1 / 2, j}}^{n}=0 \\
& v_{x_{i-1 / 2, j+1}}^{n}=0 \\
& v_{y_{i, j+3 / 2}}^{n}=0 \\
& v_{y_{i-1, j+1 / 2}^{n}}^{n}=v_{y_{i, j+1 / 2}}^{n}
\end{aligned}
$$

Figure 3-10 shows a corner/side region where all the velocities that must be set by extrapolation. This figure is easily checked by referring to the shaded velocities in Figures 3-7 through 3-9. Thus, Equation (3-2) and (3-5) again have the required behavior at corners when these extrapolated values are used. The extension to the full 3-D case is similar to the above 2-D case. 


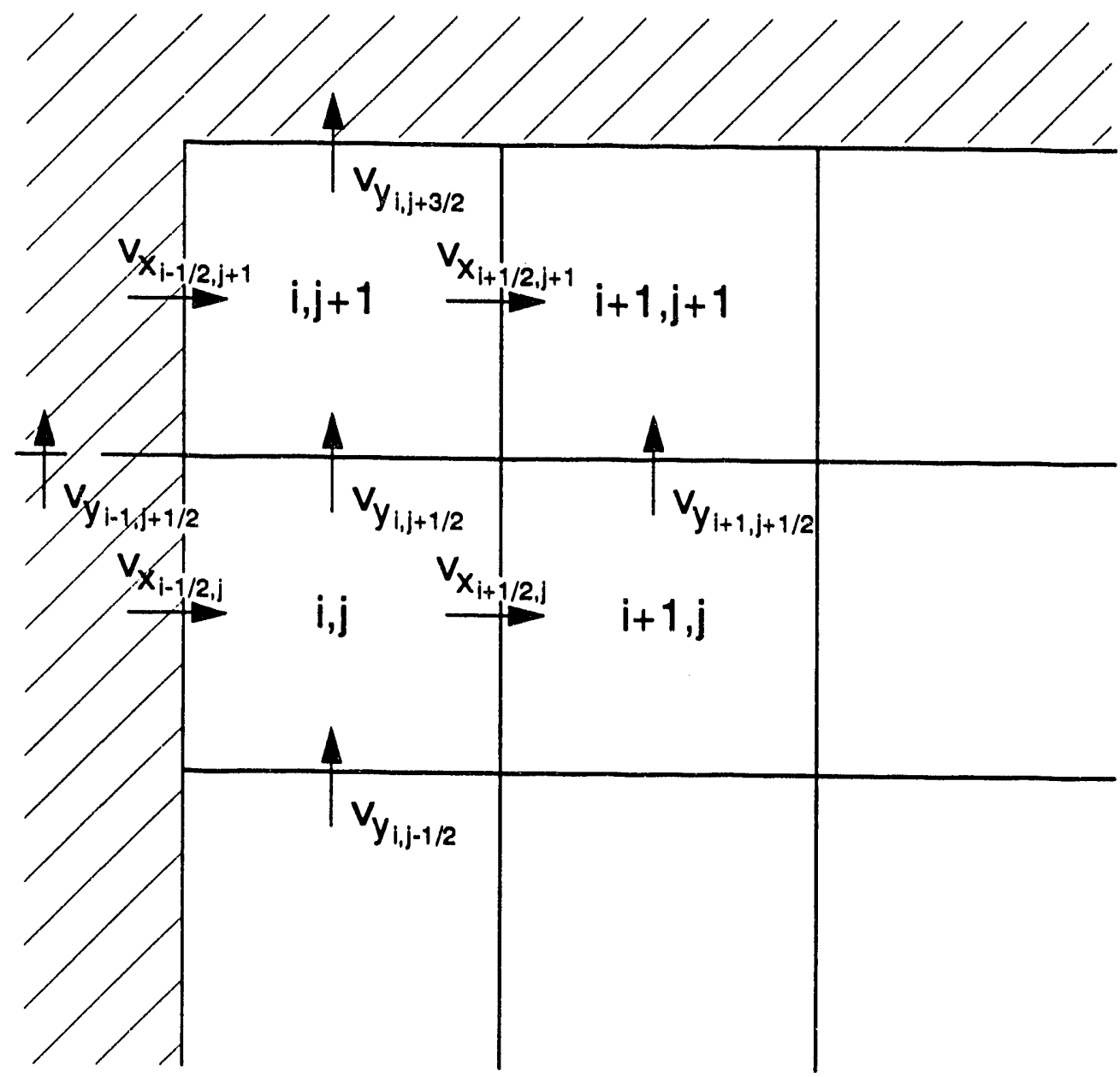

M552 rer-0482-16

Figure 3-9. Corner cell extrapolation velocities for $y$-momentum equation. 


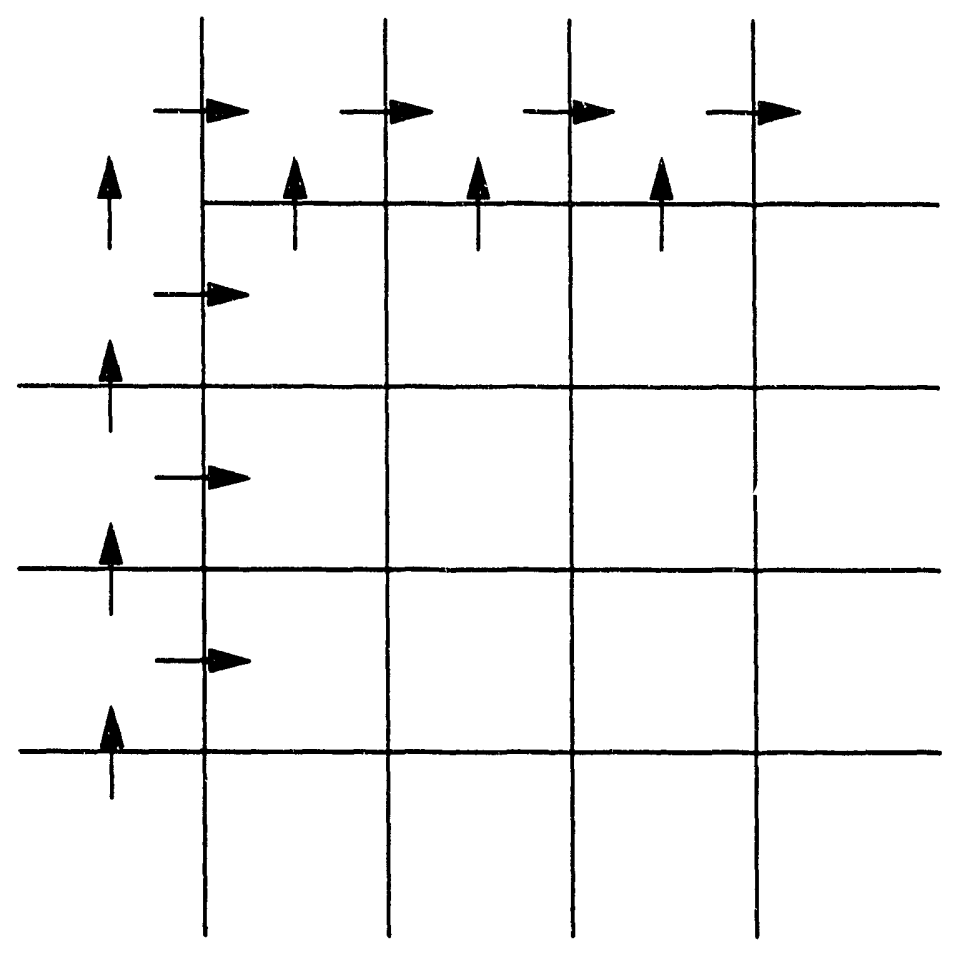

M552-WHT-492-17

Figure 3-10. Necessary extrapolations. 


\subsubsection{Terms for 1-D Connections}

It is anticipated that the case for 1-D component connections to 3-D components will be used when pipes are connected to large 3-D regions and the representation in the 3-D region is coarse. An example is the hot and cold leg connections to the core vessel when only 3 or 4 rings are used in the vesse1. In this case there is not enough resolution in the 3-D region to resolve the motion as the 1-D flow expands (inflow case) and diffuses to fill the 3-D region (see Figure 3-11) as in Figure 3-12.

There is not enough cell structure to resolve the velocity profiles shown. In this case all we can do is make sure the appropriate losses are accounted for at the inlet (or exit) junction.

\subsubsection{Pipe Connections. In Figure 3-11:}

- Junctions 6, 8, and 4, etc., are treated as normal 3-D interior junctions.

- Junctions 11 and 12 are treated as interior junctions using free slip extrapolations at the boundary wall.

- Junctions 9 and 10 are also treated as interior junctions using free slip extrapolations at the boundary.

- Junction 1 is treated as a normal 1-D junction.

We now consider the momentum equations (acceleration and pressure terms) for junctions $2,3,5$, and 7 . It should be noted that this case (1-D pipe to 3-D gross) is always used with the pipe diameter having an area less than or equal to the 3-D cell 'face area' to which it is connected. The junction at 2 may also have an orifice located at the junction cross-section. 

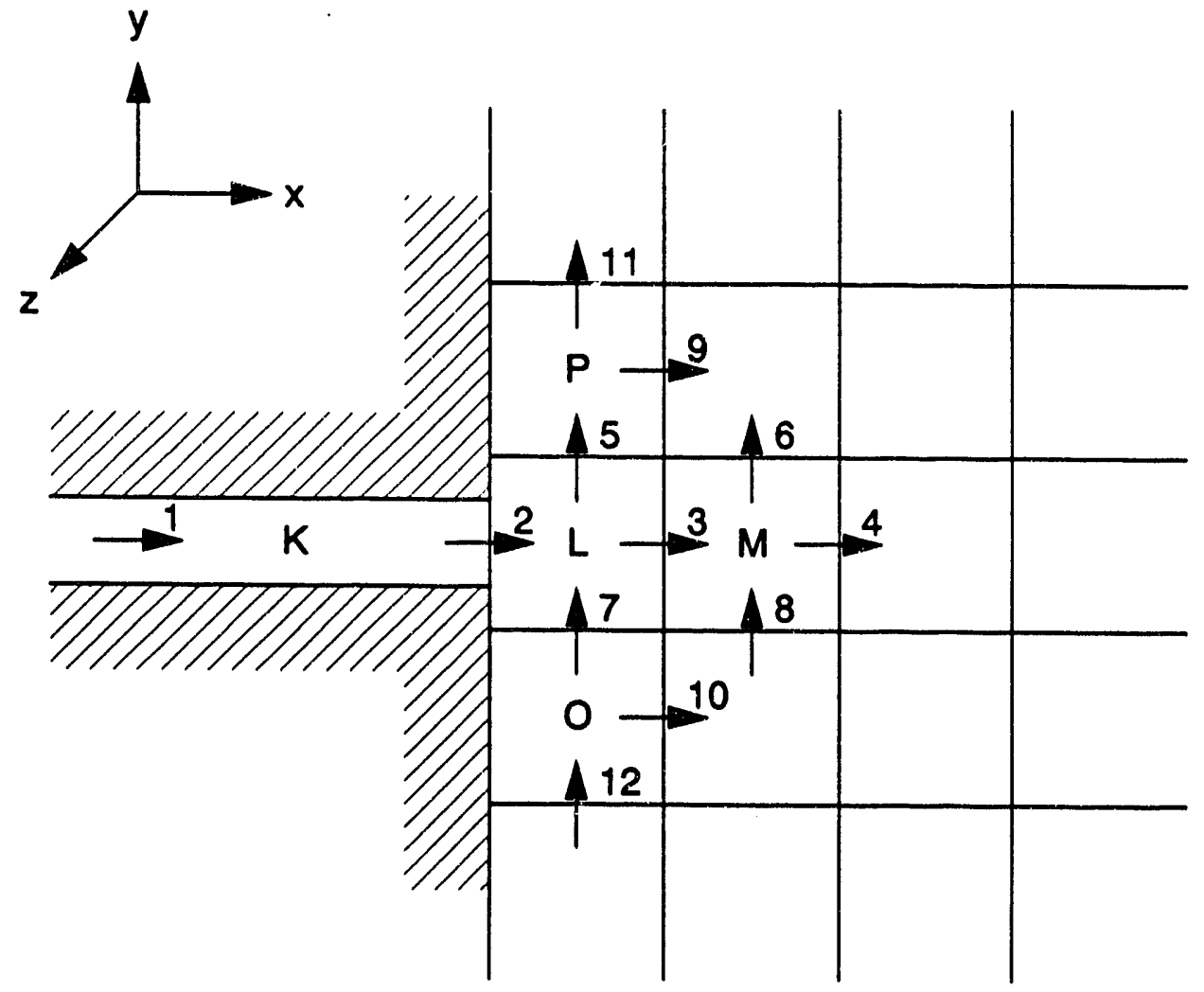

M552-WHT-492-18

Figure 3-11. Gross features, 1-D to 3-D. 

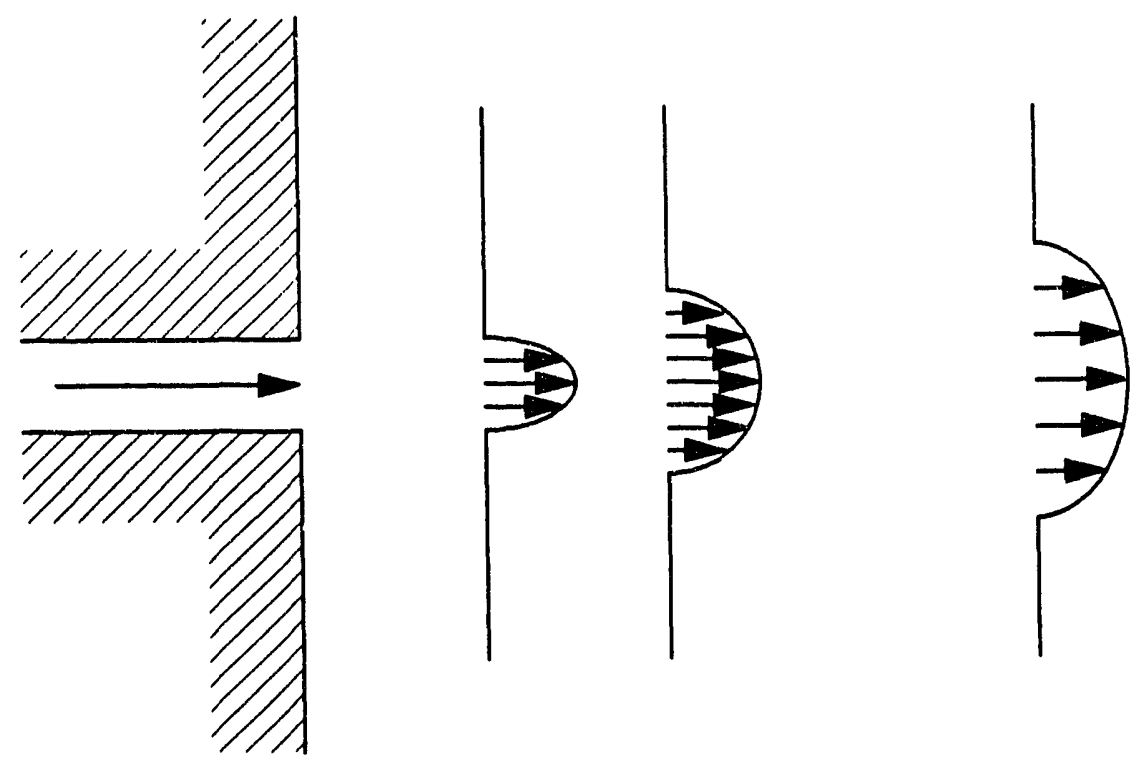

M552-WHT-492-19

Figure 3-12. Axial velocity profile, expanding flow. 
Junction 2, because of the large cell at $L$, can be treated like a 1-D junction from cell center $K$ to cell center $L$ where the cell face area should be taken as infinity in the loss calculation. This will allow the HLOSS subroutine to calculate all the appropriate loss terms. As discussed in the RELAP5 multidimensional assessment report, ${ }^{3-1}$ some problems exist in the 1-D-to-3-D connections. This approach is an interim approach, but further development and assessment is required.

The frictional terms should include only cell $K$ contributions, i.e., in the inertial and wall friction terms, 'DX' (delta length) should be treated as a zero length. Gravity terms are considered between the elevation from junction 2 to cell center $L$. The momentum flux terms from volume $L$ are set to zero, i.e., dropped from the junction 2 momentum equation because the 'axial' velocity is small in the large 3-D region i.e., like crossflows. With these changes, the regular single junction coding will be sufficient at junction 2 .

Referring to Figure 3-13, the velocities at junctions 3,5 , and 7 can now be discussed. Figure $3-13$ is exactly like Figure 3-11 except the junction velocity $v_{j 2}$ has been multiplied by the area ratio $A_{j 2} /\left(D X_{L} D Z_{L}\right)$ to scale it to an equivalent velocity $v_{1 D-3 D}$ (i.e., transporting the same mass) acting on the area $D X_{L}{ }^{*} D Z_{L}$.

The usual interior equations are formed at junctions 3,5 , and 7 . To use the usual equations at these junctions, we need $v_{1 D-3 D}$ and the extrapolated velocities shown in Figure 3-13 along the wall.

If we were at a free slip wall, then the extrapolated tangential velocities would have been $v_{5}$ and $v_{7}$ instead of $v_{5} / 2$ and $v_{7} / 2$. The reason for the half is that junction 5 sees half of the extrapolated cell as free slip and half (from the pipe) with zero tangential component. The average value is therefore $(1 / 2) v_{5}$. (Same for $v_{7}$ ).

3.2.3.2 Branch Connections. For a typical branch connection, simulating a plenum, (Figure 3-14), we envision a single large 1-D cell connected to several 3-D cells by several special junctions $(1,2,3,4$ in this figure). The junctions $1,2,3$, and 4 are multiple junctions connected to the single volume L. They may also have an orifice at any of the junction locations. 


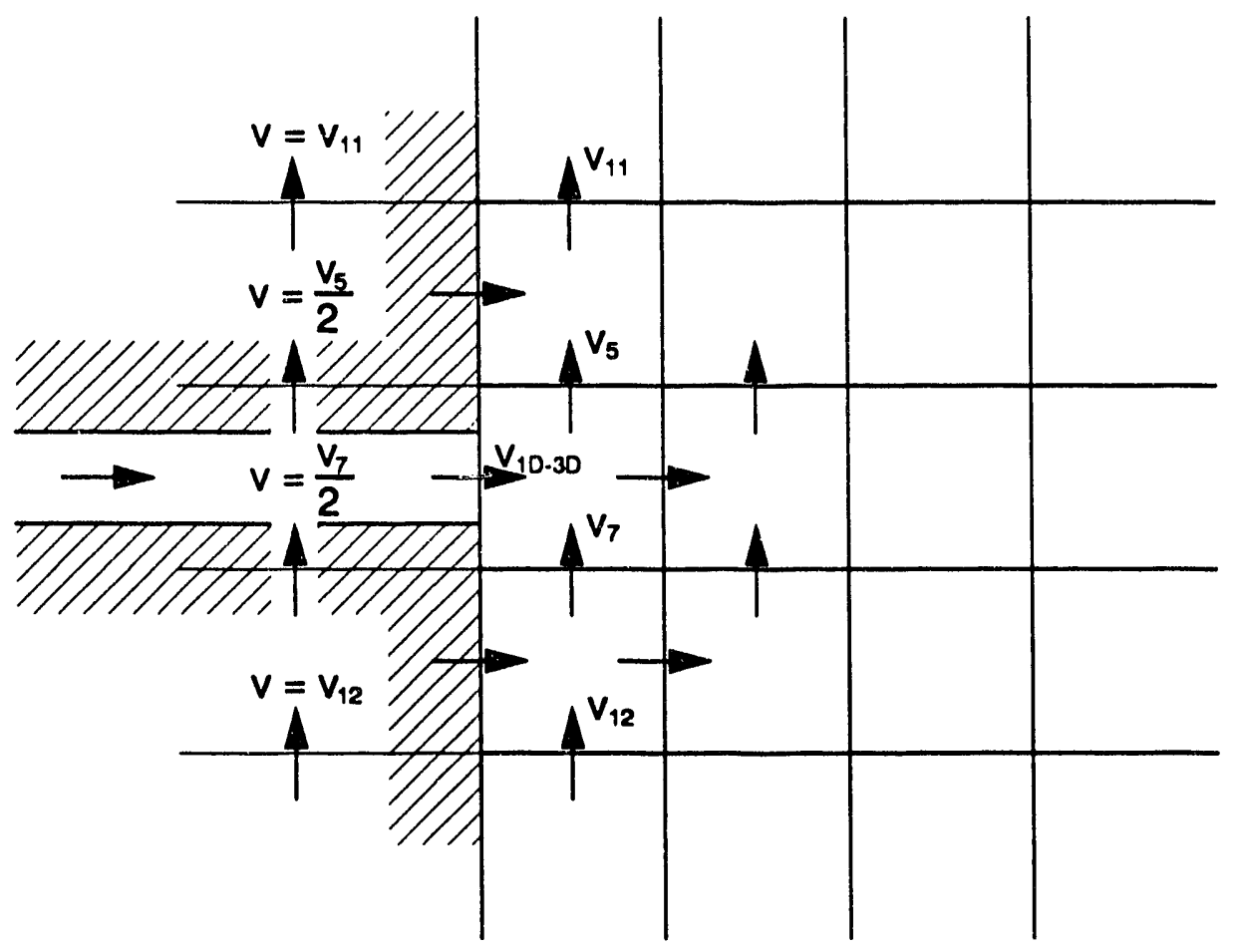

M552-WHT-492-20

Figure 3-13. Velocities needed for junctions 3, 5, and 7 . 


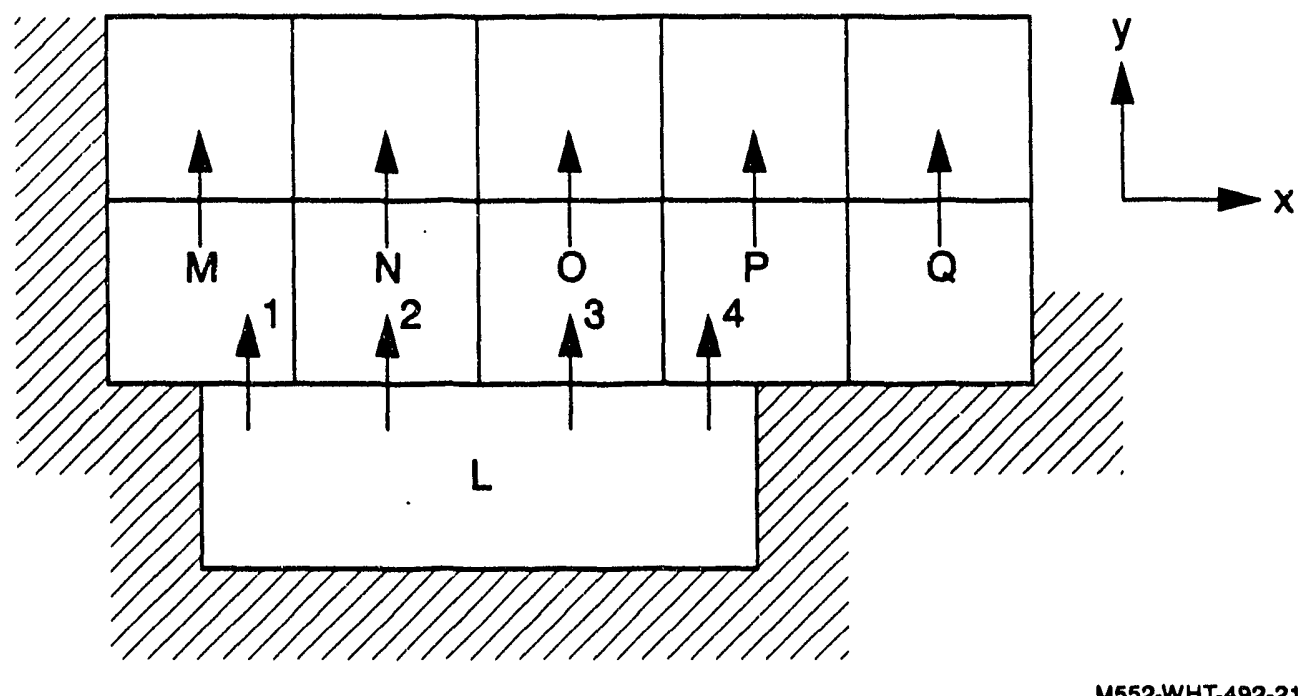

M552-WHT-492-21

Figure 3-14. Typical branch. 
The treatment of connecting junctions $1,2,3$, and 4 for a typical plenum connection is given as:

- The existing "Abrupt Area Change" model for multiple junctions on the branch volume will compute the partitioned flow areas of volume $L$ that are associated with the area change used in the loss calculation. The area change for the loss model would be calculated between the partitioned flow areas of volume $L$ and the 3-D cells (flow area equals $D X * D Z$ ) connected to volume $L$ via junctions $1,2,3$, and 4 . The loss calculation would include the effect of an orifice, if one exists. In defining the junction areas, they should add up to the flow area of volume $L$, and be partitioned appropriately to each junction.

- Al1 momentum flux calculations at junctions $1,2,3$, and 4 , or at least the contribution from the $3-D$ volumes (including $L$ cell contributions) would be dropped, i.e., like crossflow junctions. Also, all the flux terms (they are small in the plenum volume since large area implies small velocity) would be dropped.

- All other terms should be calculated as is done for a regular junction.

Figure 3-15 is exactly like Figure 3-14, except the junction velocities $v_{j 1}, v_{j 2}, v_{j 3}$, and $v_{j 4}$ have been multiplied by the area ratios:

$$
\frac{A_{j 1}}{D X_{M} D Z_{M}} \quad \frac{A_{j 2}}{D X_{N} D Z_{N}} \quad \frac{A_{j 3}}{D X_{0} D Z_{0}} \quad \frac{A_{j 4}}{D X_{P} D Z_{P}}
$$

to scale them to equivalent velocities acting on the 3-D areas. After being scaled, the velocities are called $v_{31}, v_{32}, v_{33}$, and $v_{34}$.

If the normal 3-D interior equations are used then the shaded velocities must be known. They should be "extrapolated" with the values shown on the figure. 


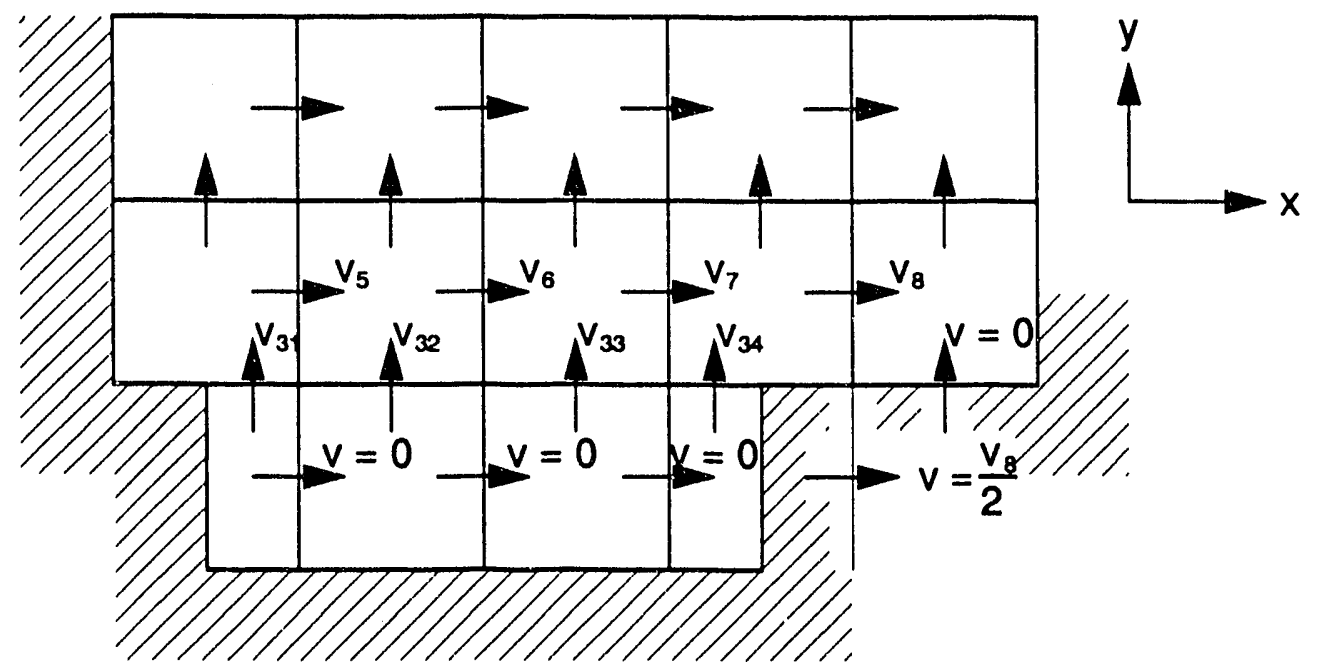

M552-WHT-492-22

Figure 3-15. Extrapolated velocities for a plenum. 
If the normal 3-D interior equations are used then the shaded velocities must be known. They should be "extrapolated" with the values shown on the figure.

All tangential velocities that are inside the plenum are extrapolated as zero velocities. The first tangential velocity outside the plenum is extrapolated as $1 / 2$ the value of the corresponding inside value. If further tangential extrapolations are needed for junctions further from the plenum, the free slip extrapolation is used.

\subsection{Cylindrical Coordinates}

The spatial gradient terms of the momentum flux terms are given in Equation (2-18), and are repeated here for reference. They are:

$$
\begin{aligned}
& v_{r} \frac{\partial v_{r}}{\partial r}+\frac{v_{\theta}}{r} \frac{\partial v_{r}}{\partial \theta}-\frac{v_{\theta}^{2}}{r}+v_{z} \frac{\partial v_{r}}{\partial z}, \\
& v_{r} \frac{\partial v_{\theta}}{\partial r}+\frac{v_{\theta}}{r} \frac{\partial v_{\theta}}{\partial \theta}+\frac{v_{r} v_{\theta}}{r}+v_{z} \frac{\partial v_{\theta}}{\partial z} \text {, and } \\
& v_{r} \frac{\partial v_{z}}{\partial r}+\frac{v_{\theta}}{r} \frac{\partial v_{z}}{\partial \theta}+v_{z} \frac{\partial v_{z}}{\partial z},
\end{aligned}
$$

where there are additional acceleration terms that were not in the Cartesian coordinate formulations,

$$
-\frac{v_{\theta}{ }^{2}}{r} \text { and } \frac{v_{r} v_{\theta}}{r} \text {. }
$$

The finite difference form and implementation of these equations are given in the next section.

\subsubsection{Momentum Flux Terms}

The finite difference form of the flux terms are given in the same format as before. Also given here are the discussions on special consideration or treatment of some terms of the spatial fluxes. 
The $r$-face finite difference flux term for the momentum cell centered on face $i+1 / 2, j, k$, Figure $3-16$, is

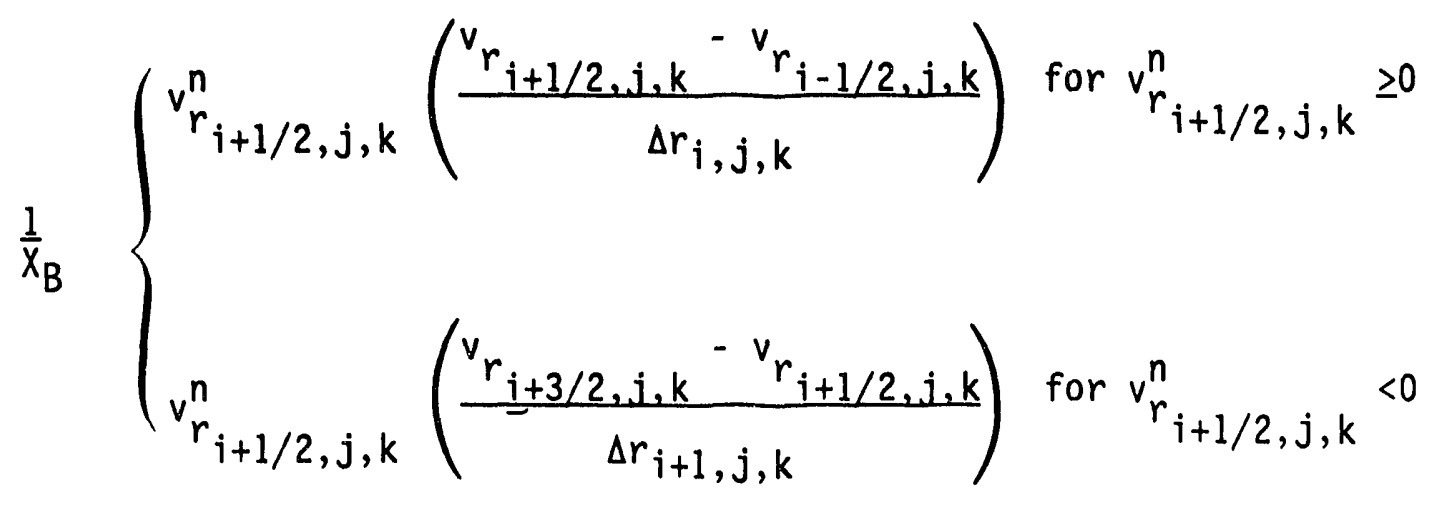

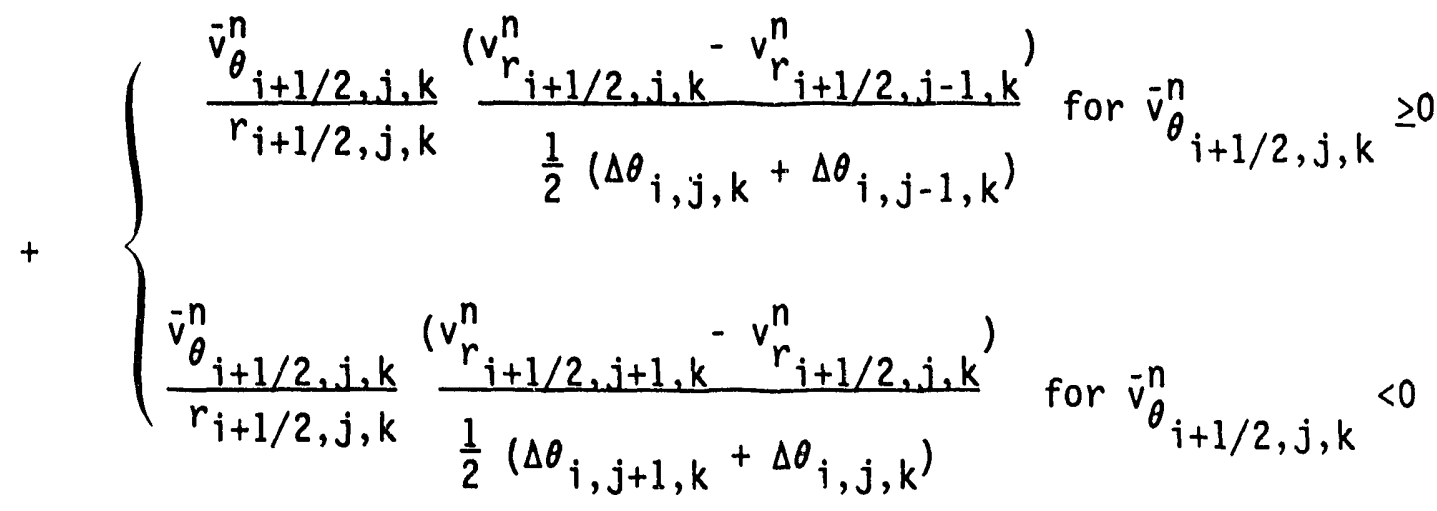

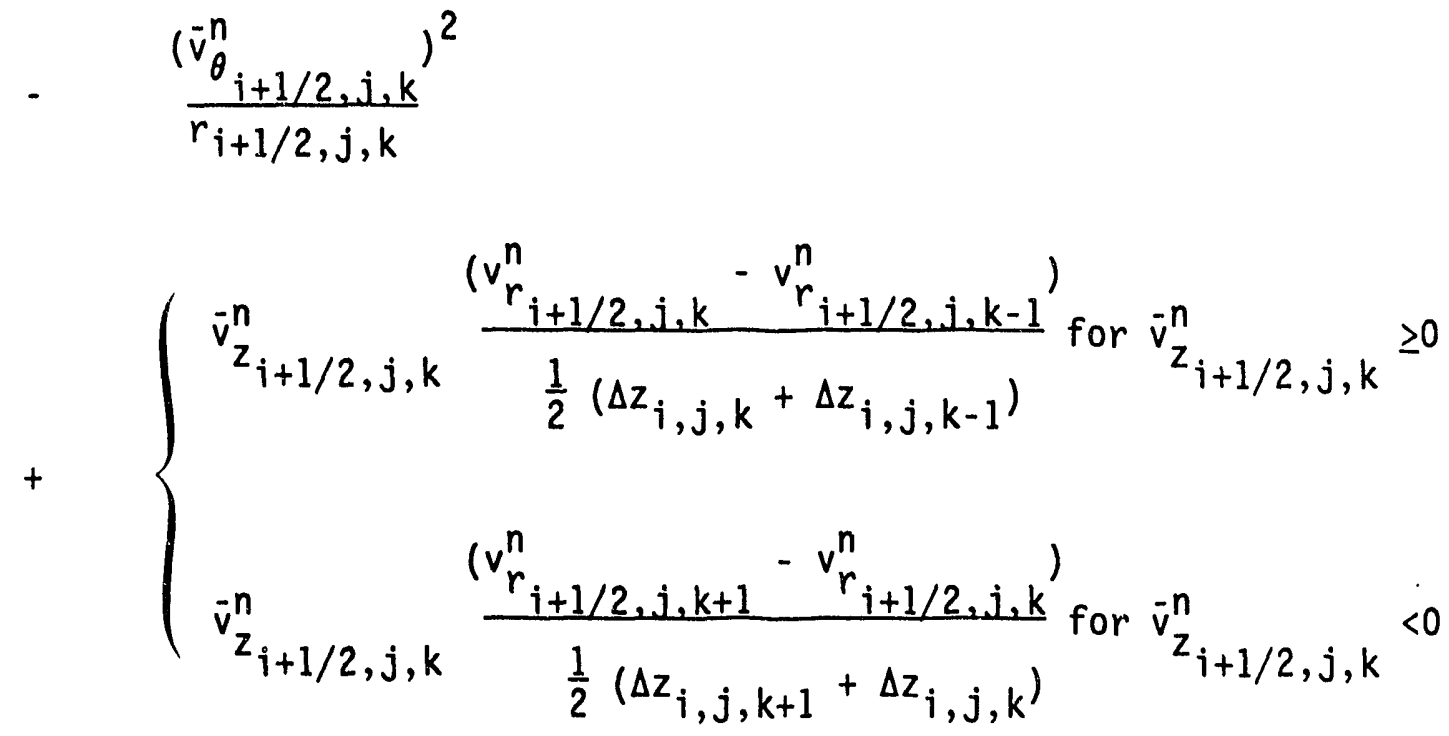

where

$\bar{v}_{\theta_{i+1 / 2, j, k}}^{-n}=\frac{1}{4}\left(v_{\theta_{i, j+1 / 2, k}}^{n}+v_{\theta_{i, j-1 / 2, k}^{n}}+v_{\theta_{i+1, j+1 / 2, k}^{n}}+v_{\theta_{i+1, j-1 / 2, k}^{n}}\right)$ 


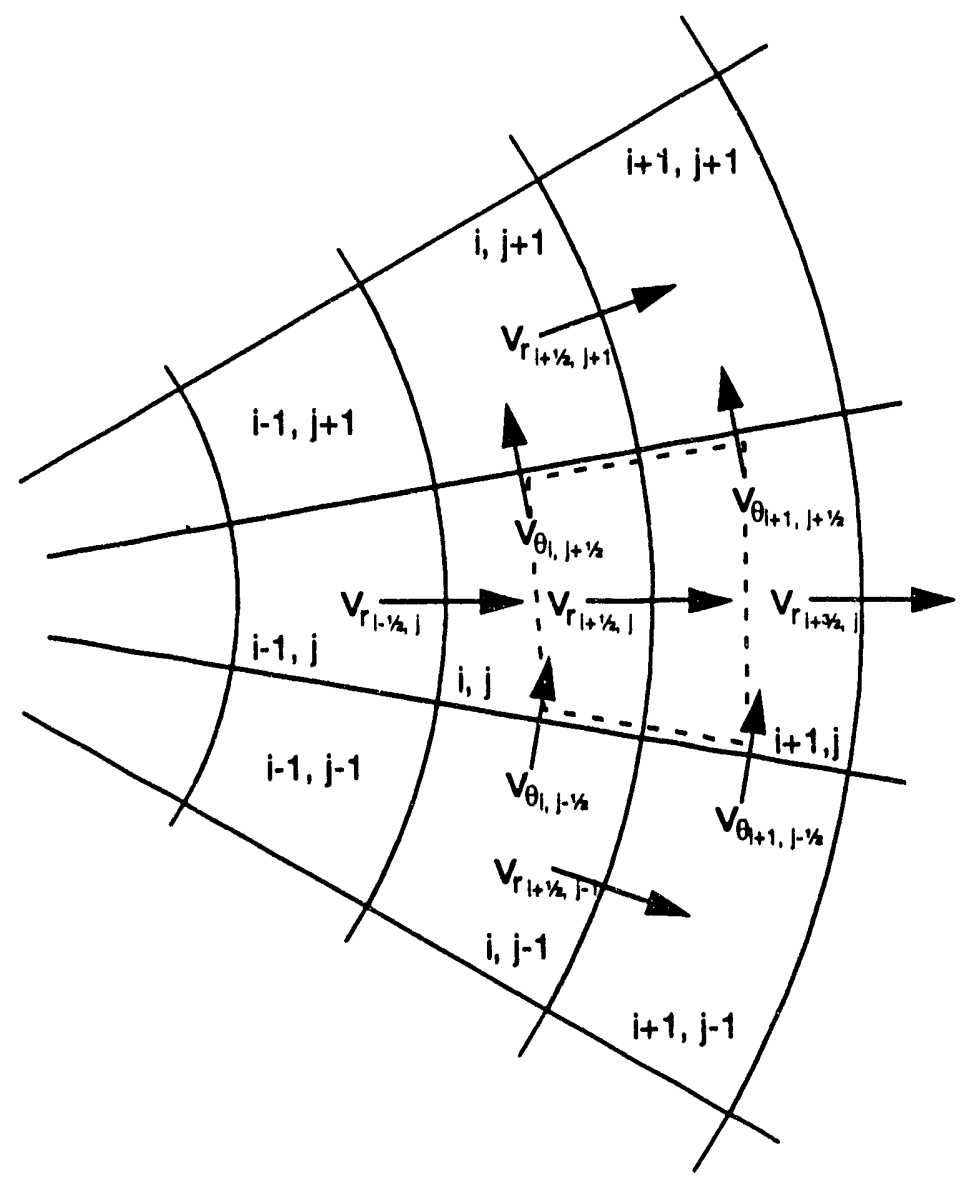

M552-WHT-492-23

Figure 3-16. Control volume for the momentum cell about $v_{r_{i+1 / 2, j, k}}$. 


$$
v_{z_{i+1 / 2, j, k}}^{n}=\frac{1}{4}\left(v_{z_{i, j, k+1 / 2}^{n}}^{n}+v_{z_{i, j, k-1 / 2}^{n}}^{n}+v_{z_{i+1, j, k+1 / 2}^{n}}^{n}+v_{z_{i+1, j, k-1 / 2}^{n}}^{n}\right)
$$

and

$$
X_{B}=2 \frac{A_{i+1 / 2}-A_{i-1 / 2}}{A_{i+1 / 2}^{2} A_{i-1 / 2}} \frac{A_{i+1}^{2} A_{i}^{2}}{A_{i+1}^{2}-A_{i}^{2}} \frac{r_{i+1}-r_{i}}{r_{i+1 / 2}-r_{i-1 / 2}} \text { for } v_{r_{i+1 / 2}}>0
$$

is a Bernoulli correction factor based on the area variation between sequential radial hydrodynamic volumes and the junctions connecting them. $X_{B}$ can be determined from the following development.

In pure steady incompressible single phase radial flow (without gravity and losses due to friction) with no variation in the $\theta$-direction and $z$-direction, the radial momentum equation ( $r$ component of Equation (2-20)) simplifies to

$$
\rho v_{r} \frac{\partial v_{r}}{\partial r}=-\frac{\partial P}{\partial r}
$$

which can also be written as

$$
\rho \frac{\partial\left(\frac{1}{2} v_{r}^{2}\right)}{\partial r}=-\frac{\partial P}{\partial r}
$$

The finite difference form of the radial momentum Equation (3-31) for flow in the positive $r$-direction, in this case (see Figure $3-17$ ), is given by

$$
\rho v_{r_{i+1 / 2}} \frac{\left(v r_{i+1 / 2}-v r_{i-1 / 2}\right)}{r_{i+1 / 2}-r_{i-1 / 2}}=-\frac{\left(P_{i+1}-P_{i}\right)}{r_{i+1}-r_{i}}
$$

This finite difference form of Equation (3-31) does not exactly match the finite difference form (Bernoulli equation) of Equation (3-32). Equation (3-33) can be made the same as the finite difference form of Equation (3-32), if it is written as 


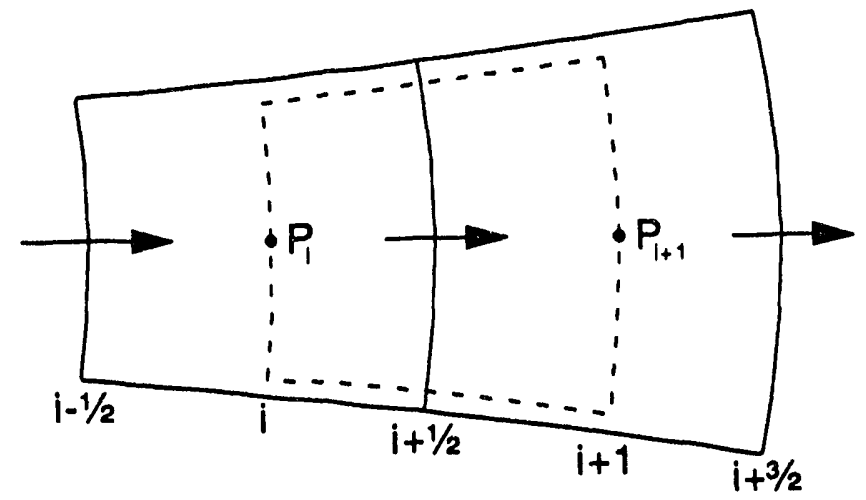

M552-WHT-492-24

Figure 3-17. Control volume for Bernoulli correction. 


$$
\frac{\rho v_{i+1 / 2}}{x_{B}} \frac{\left(v_{r_{i+1 / 2}}-v_{r_{i-1 / 2}}\right)}{r_{i+1 / 2}-r_{i-1 / 2}}=-\frac{\left(p_{i+1}-p_{i}\right)}{r_{i+1}-r_{i}}
$$

Solving for $X_{B}$ when radial flow is constant, gives Equation (3-30).

The factor for reverse flow is similar. This correction factor is always applied on the radial finite difference terms in Equation (3-27).

The $\theta$-face finite difference flux term over the control volume shown in Figure $3-18$ is

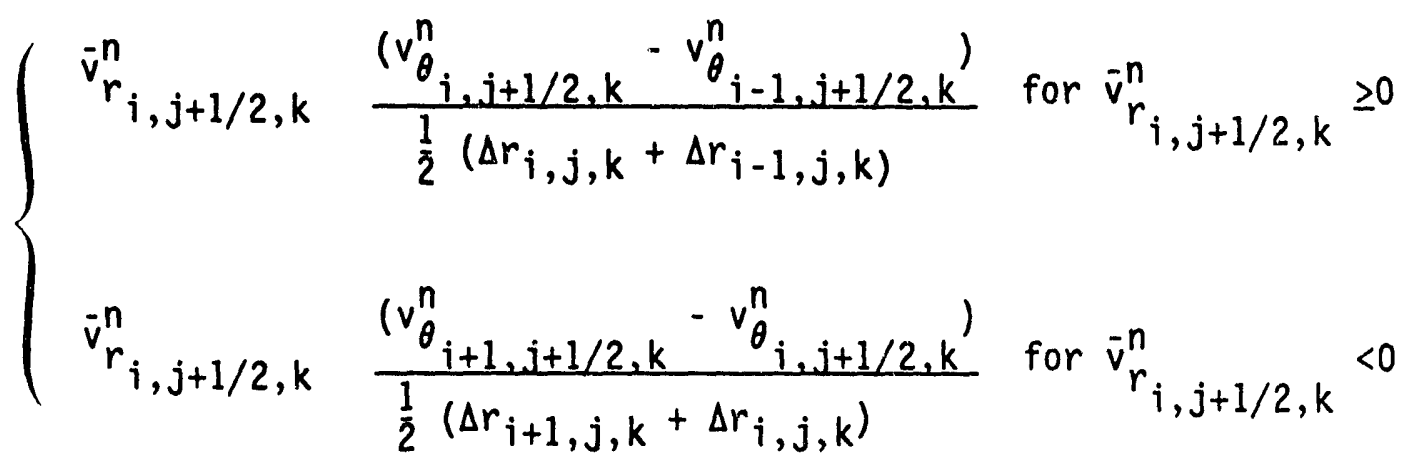

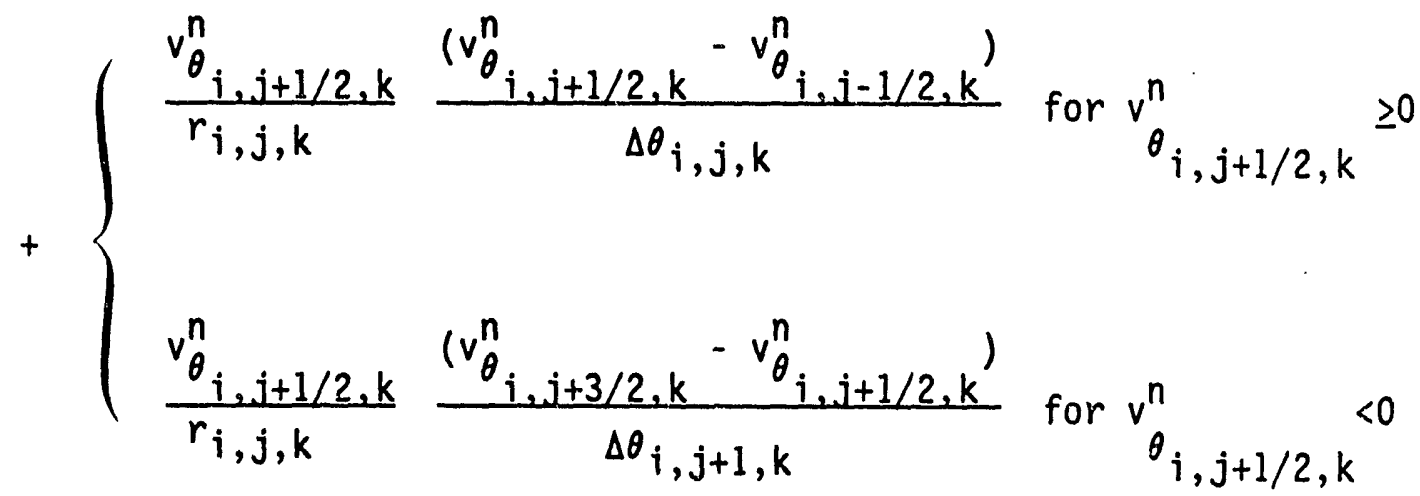

$$
\begin{aligned}
& +\left\{\begin{array}{l}
\left.\frac{v_{r_{i, j+1 / 2, k}}^{n} \frac{1}{2}\left(v_{\theta}^{n}\right.}{\frac{1}{2}\left(r_{i-1, j, k}+r_{i, j, k}\right)}+v_{\theta_{i, j+1 / 2, k}}^{n}\right) \\
\frac{\bar{v}_{r_{i, j+1 / 2, k}}^{n}\left(v_{\theta_{i, j+1 / 2, k}}^{n}+v_{\theta}^{n}\right.}{\frac{1}{2}\left(r_{i, j, k}+r_{i+1, j, k}\right)} \text { for } v_{r_{i, j+1 / 2, k}}^{n} \geq 0
\end{array}\right. \\
& \text { 3-32 }
\end{aligned}
$$




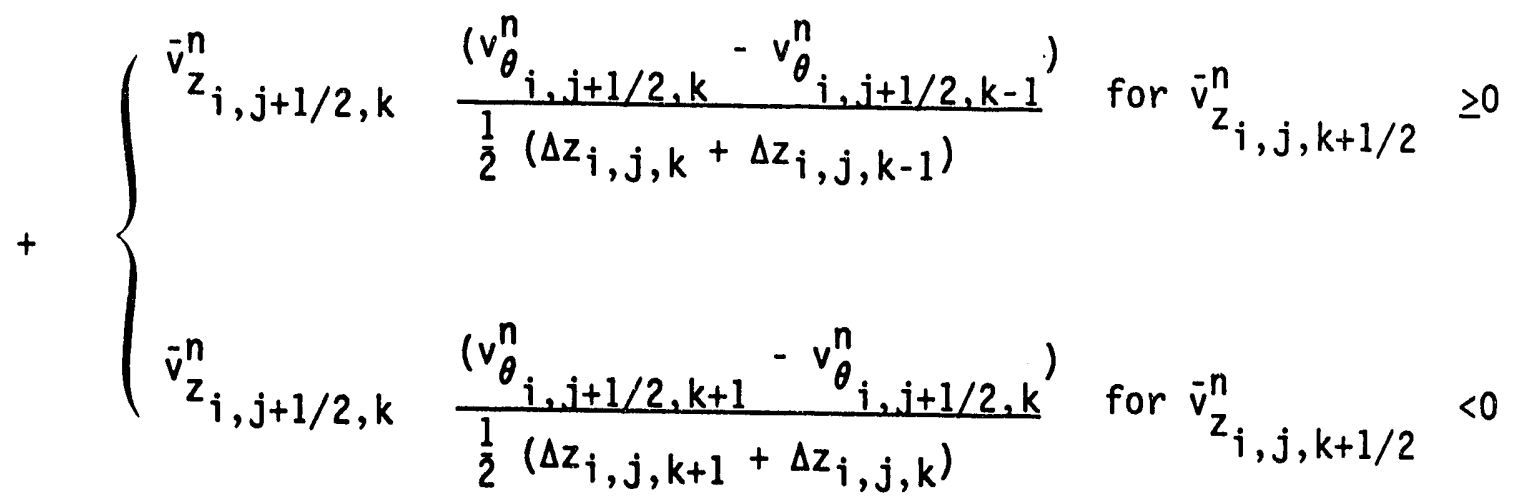

where

$$
\bar{v}_{r_{i, j+1 / 2, k}}^{n}=\frac{1}{4}\left(v_{r_{i+1 / 2, j, k}}^{n}+v_{r_{i-1 / 2, j, k}}^{n}+v_{r_{i+1 / 2, j+1, k}}^{n}+v_{r_{i-1 / 2, j+1, k}}^{n}\right)^{\prime}
$$

and

$$
v_{z_{i, j, k+1 / 2}}^{n}=\frac{1}{4}\left(v_{z_{i, j, k+1 / 2}^{n}}^{n}+v_{z_{i, j, k-1 / 2}^{n}}^{n}+v_{z_{i, j+1, k+1 / 2}^{n}}+v_{z_{i, j+1, k-1 / 2}^{n}}\right)
$$

The $\frac{v_{r} v_{\theta}}{r}$ acceleration term uses an azimuthal velocity and radius at the upwind edge of the control volume. This was done as a result of asymmetric behavior noted an $r-\theta$ symmetric flow test problem run during the developmental assessment. ${ }^{3-1}$ The problem is shown in the following analysis.

In pure steady incompressible single phase radial and azimuthal flow (without gravity and losses due to friction) with no variation in the $\theta$ direction and $z$ direction, the radial and azimuthal momentum equations ( $r$ component and $\theta$ component of Equation (2-20)) simplify to

$$
\begin{aligned}
& r: \quad v_{r} \frac{\partial v_{r}}{\partial r}-\frac{v_{\theta}^{2}}{r}=-\frac{1}{\rho} \frac{\partial P}{\partial r} \\
& \theta: \quad \frac{\partial v_{\theta}}{\partial r}=-\frac{v_{\theta}}{r}
\end{aligned}
$$




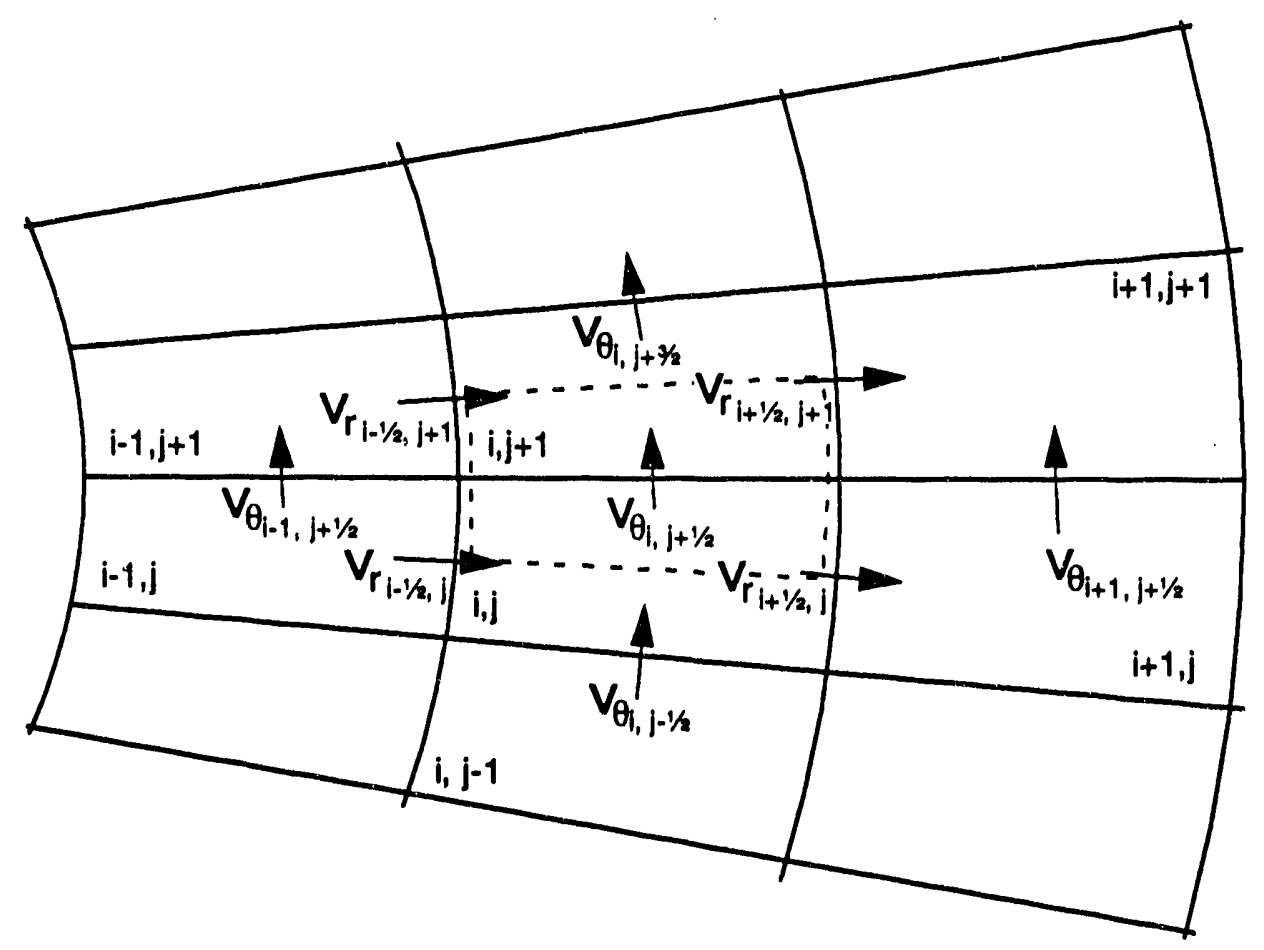

M552-WHT-482-25

Figure 3-18. Control volume for the momentum cell about $v_{\theta_{i, j+1 / 2, k}}$. 
For flow in the negative $r$ direction (radial flow), using the nomenclature shown in Figure 3-19, we can write the $\theta$ finite. difference momentum equation as

$$
\frac{v_{\theta_{i+1}}-v_{\theta_{i}}}{r_{i+1}-r_{i}}=-\frac{v_{\theta_{i}}}{r_{i}}
$$

and rearranging yields

$$
v_{\theta_{i}}=\frac{v_{\theta_{i+1}}}{1-\frac{r_{i+1}-r_{i}}{r_{i}}}
$$

As $r_{i}$ decreases, $v_{\theta}$ changes sign. By using an upwind average velocity and average radius, the difference equation

$$
\frac{v_{\theta_{i+1}}-v_{\theta_{i}}}{r_{i+1}-r_{i}}=-\frac{1}{2} \frac{\left(v_{\theta_{i+1}}+v_{\theta_{i}}\right)}{\frac{1}{2}\left(r_{i+1}+r_{i}\right)}
$$

reduces to

$$
v_{\theta_{i}}=v_{\theta_{i+1}} \frac{r_{i+1}}{r_{i}}
$$

which is the expected result from continuity.

Lastly, the $z$-face finite difference flux terms centered at $v_{z_{i, j, k+1 / 2}}{ }^{i s}$

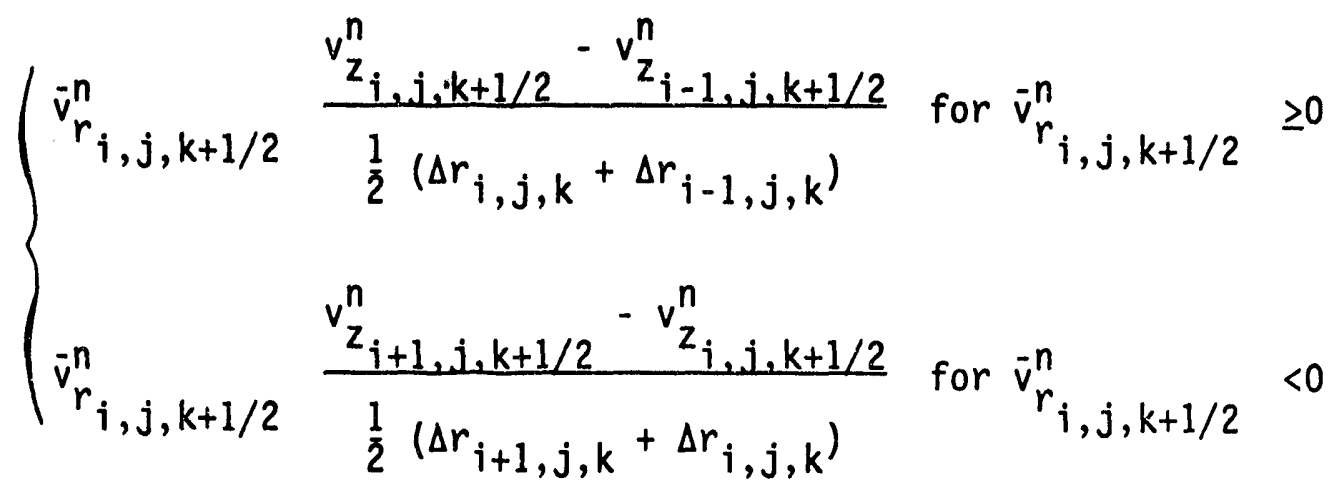

$$
\begin{aligned}
& \text { 3-35 }
\end{aligned}
$$




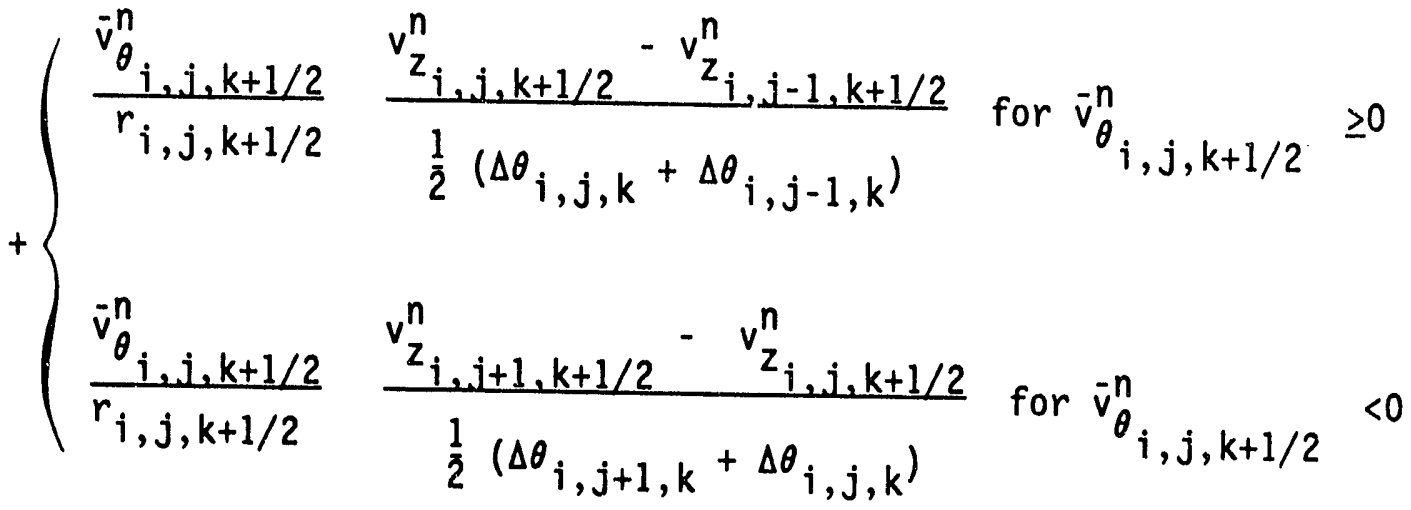

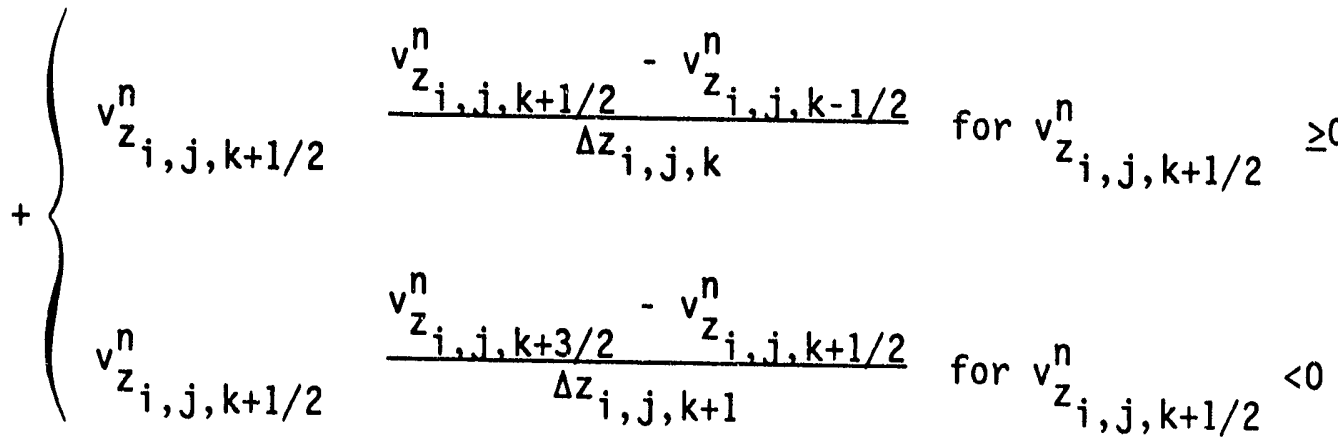

where

$$
v_{r_{i, j, k+1 / 2}}^{n}=\frac{1}{4}\left(v_{r_{i-1 / 2, j, k}}^{n}+v_{r_{i+1 / 2, j, k}}^{n}+v_{r_{i-1 / 2, j, k+1}}^{n}+v_{r_{i+1 / 2, j, k+1}^{n}}^{n}\right)
$$

and

$$
v_{\theta_{i, j, k+1 / 2}^{n}}^{n}=\frac{1}{4}\left(v_{\theta_{i, j-1 / 2, k}^{n}}+v_{\theta_{i, j+1 / 2, k}^{n}}+v_{\theta_{i, j-1 / 2, k+1}^{n}}+v_{\theta_{i, j+1 / 2, k+1}^{n}}\right)
$$

\subsubsection{Terms at Interior Boundaries}

For the cylindrical geometry case, interior walls and corners are treated the same as in the Cartesian case, as described in Section 3.2.2. However, there is a special case that arises in the cylindrical geometry that requires special treatment. This case is the center of the cylindrical region. 


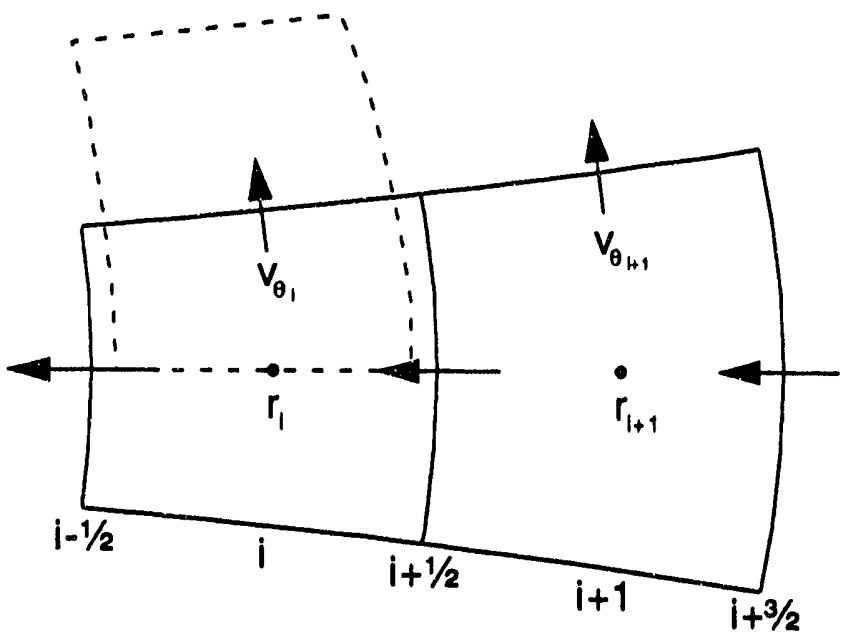

M552-WHT-492-26

Figure 3-19. Control volume for cell at $v_{\theta_{i}}$. 
In the finite difference scheme a singularity arises at $r=0$ in cylindrical coordinates. At this point, radial, azimuthal, and axial velocities do not exist. In order to perform the finite differencing of the control volume neighboring the singularity, the following methods are employed. The radial momentum for the control volume shown in Figure 3-20 requires a velocity $\left(v_{r_{0}}\right)$ at $r_{0}(r=0)$ to use in the radial convective term. The velocity used is

$$
v_{r_{0}}=\frac{v_{r}-v_{r}^{-}}{2} \quad \text { for } v_{r}>0
$$

where $v_{r_{0}}$ is the velocity used at $r_{0}$, and $v_{r}^{-}$and $v_{r}$ are the radial velocities at $r$ shown in Figure 3-20. Hence, the convective difference term in the radial momentum equation becomes

$$
v_{r} \frac{\partial v_{r}}{\partial r}=v_{r}\left(\frac{v_{r}-v_{r_{0}}}{\Delta r}\right)
$$

where

$$
\Delta r=r-r_{0}=r
$$

The differencing for the radial term in the azimuthal momentum equation is similar and the velocities used are shown in Figure 3-21,

$$
v_{r} \frac{\partial v_{\theta}}{\partial r}=v_{r}\left(\frac{v_{\theta}+v_{\theta}^{-}}{\Delta r}\right) \quad \text { for } v_{r}>0
$$

where

$$
v_{r}=\frac{\left(v_{r}^{-}+v_{r_{0}}^{-}\right)+\left(v_{r}^{+}+v_{r_{0}}^{+}\right)}{4}
$$

Similar techniques are used for the axial finite difference equations.

Specifying $r_{0}>0$ in the input to the multidimensional model creates a cylindrical wall at the center of the geometry. This wall is treated the same as other wall boundaries previously discussed. 


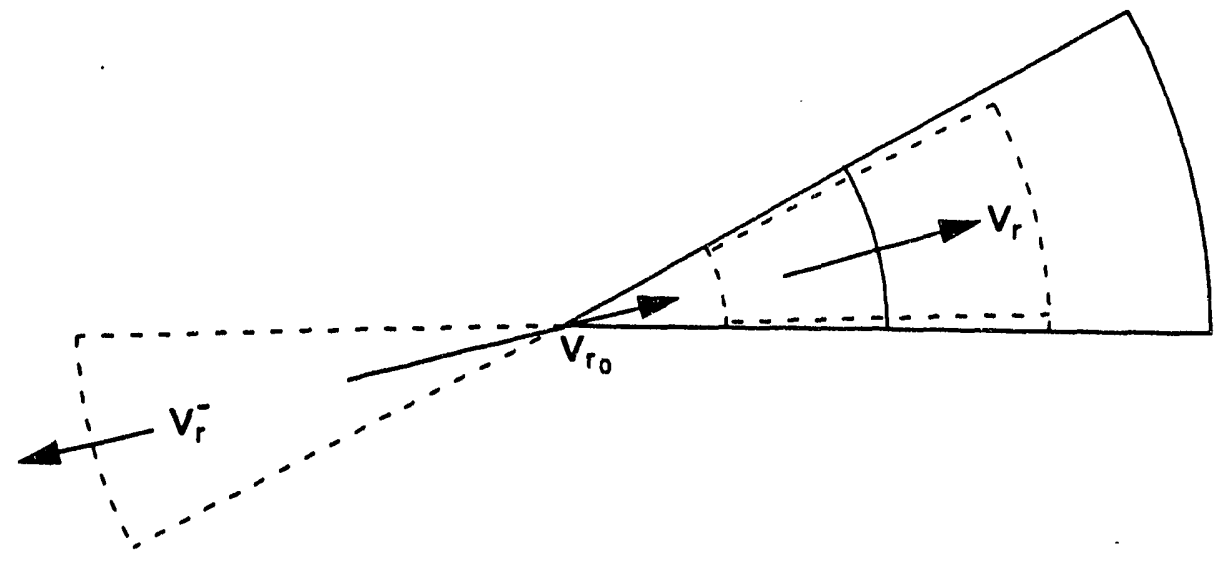

M552-WHT-492-27

Figure 3-20. Control volume for radial momentum flux. 


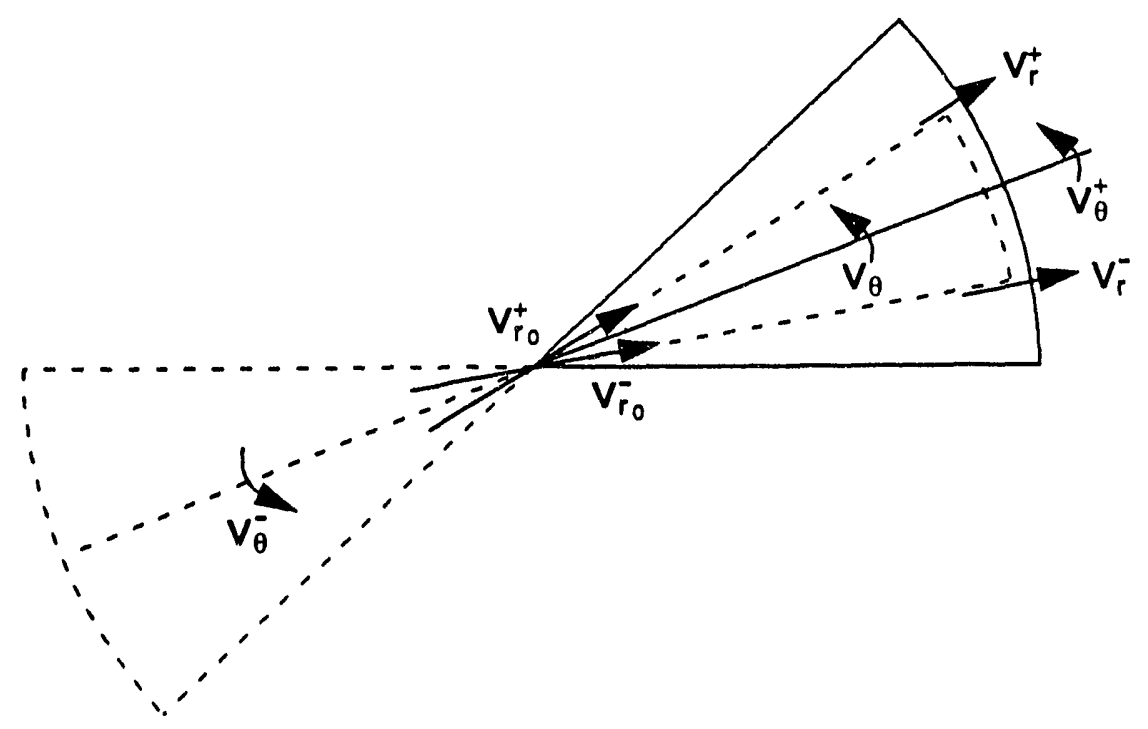

M552-WHT-492-28

Figure 3-21. Control volume for azimuthal momentum flux. 


\subsubsection{Terms for 1-D Connections}

The 1-D connections to a 3-D cylindrical geometry are discussed in this section. The radial connections are given first, then the $\theta$-connections are discussed, followed by the axial connections.

The radial connections are treated differently depending on the location of the connection, either at the center or the outer ring. For the center, or inner ring, the extrapolated velocities that are perpendicular to the radial direction are unchanged. This is independent of the face connections of the 1-D to 3-D junction.

For connections to the outer face of the outer ring, the extrapolated velocities perpendicular to the $r$-direction are modified by reducing their magnitude as a function of the junction face areas. The extrapolated velocity is:

$v_{\theta_{i+1, j+1 / 2}}=\frac{1}{2} \frac{\left(A_{i+1 / 2, j}-A_{j}\right)}{A_{i+1 / 2, j}}+\frac{1}{2} \frac{\left(A_{i+1 / 2, j+1}-A_{j+1}\right)}{A_{i+1 / 2, j+1}} v_{\theta_{i, j+1 / 2}}$

as shown in Figure 3-22, where $A_{j}$ and $A_{j+1}$ are the junction areas of the external connections, and $A_{i+1 / 2, j}$ and $A_{j+1 / 2, j+1}$ are the $r$-face areas of the surface of the multidimensional component. This is performed only for the junctions connected to the exit $r$-face of the outer ring.

Connections to $\theta$-faces are not accounted for in the 3-D momentum flux terms. Therefore, crossflow types of connections must be specified.

Connections to $z$-faces by 1-D junctions sets the extrapolated velocities surrounding the $z$-direction to zero. At this point, the only testing of $z$-face connections has been with plenums, hence the appropriate boundary condition. 


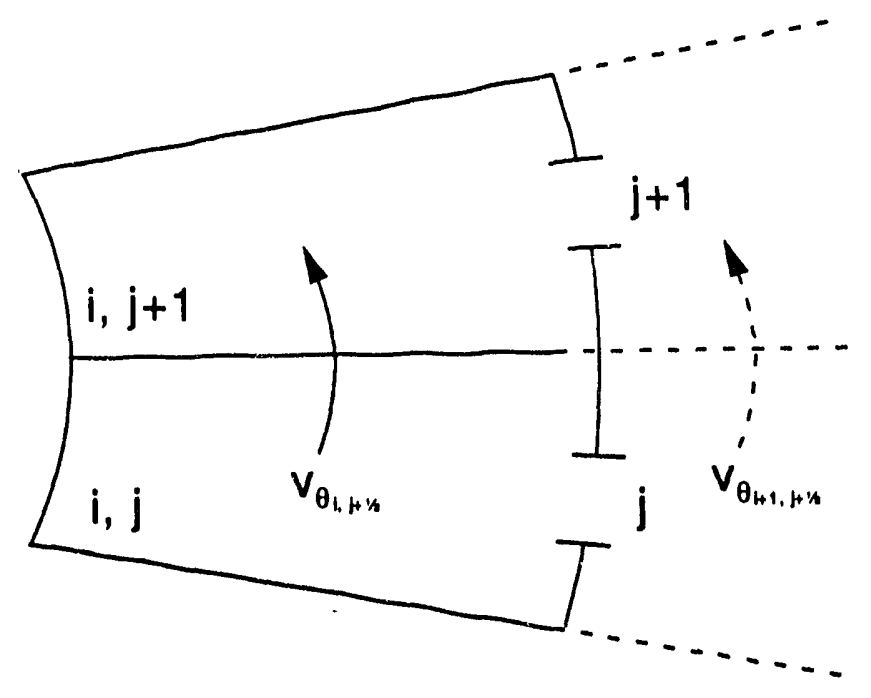

M552-WHT-482-29

Figure 3-22. External connections in $r-\theta$ geometry. 
At this point, all connections made to interior cells of a 3-D region are not accounted for in the 3-D momentum flux calculations. For system modeling purposes, crossflow connections should be used at interior faces/cells of the multidimensional component.

As discussed in the RELAP5 multidimensional assessment report, $3-1$ some problems exist for the 1-D-to-3-D connections. This approach is an interim approach, but further development and assessment are required.

\subsection{Programming Constderations}

The implementation of the 3-D model into the RELAP5 code started in January 1985 with the implementation of the 3-D model ${ }^{3-2}$ into the ATHENA ${ }^{3-3}$ code. The original model had three constraints applied in its development in addition to its stated goals. They were: (1) use the dynamic storage philosophy currently in ATHENA, (2) minimal storage usage, and (3) apply vectorization techniques to the model. Similar goals were used during FY-91 for implementing the 3-D model into RELAP5.

The dynamic storage and the extensive use of the "FTB" memory management used by RELAP5 precluded a normal installation of a 3-D model into the code. The problems encountered consist of how and where to store information for the 3-D advancement variables since minimal storage requires the existing 1-D volume and junction variables to be used where possible, and new storage for other variables. Thus, the approach taken was to make the 3-D component have its own storage block and use pointers and offsets to transfer information between the volume, junction, and 3-D variable storage blocks.

The storage structure for the multidimensional component includes locations for the extrapolated normal and tangential velocities. These locations are called pseudo-cells and contain either the extrapolated or externally connected velocities. 


\subsection{Routines}

The routines for the 3-D component are listed below with appropriate comments concerning their purpose.

FLUX3D. This is the main routine for calculating the 3-D momentum flux terms. It is called from VEXPLT at a point where the momentum flux terms are being calculated. Two arguments are passed, the index to the momentum flux terms (sum and difference form), and the component index to the 3-D component.

The first operation is to zero out the 3-D component storage for the velocities. Then, the inverted junction table indices are advanced to the first volume in the 3-D component.

Now, the inverted junction table can be used to set the 3-D junction velocities. The procedure is similar to the volume average velocity calculation, i.e., loop over inlets and outlets of the volume, except now the inlet and outlet velocities are stored in their respective $x$, $y$, and $z$ locations instead of being summed. Additionally, the necessary velocity extrapolations for the normal and tangential velocity boundary conditions are set, as well as the external connecting velocities. The initial zero out of the array sets the normal velocities $(v=0)$, while the above second step sets all the others (tangential, interior, and exterior).

The internal, side, corner, and external velocities are now loaded into the 3-D velocity array so that Equations (3-2) through (3-4), (3-6) through (3-7), and (3-8) through $(3-10)$ are evaluated. These terms are stored in CONVF and CONVG for the liquid and vapor flux terms at each defined (in the 3-D component) junction, and are transferred back to the calling routine VEXPLT.

VEXPLT. This existing subroutine calculates the momentum equations by combining various terms, including the momentum flux terms. This routine was modified to call the subroutine FLUX3D where the momentum flux terms for the 3-D component are returned in the subscripted 
variables CONVF and CONVG. Upon returning to VEXPLT, CONVF and CONVG are used to calculate CONVS and CONVD for use in the sum and difference momentum equations.

R3DCMP. This subroutine reads the RELAP5 input for the 3-D component. The currently available options are for uniform input. Processing duties include calculating storage requirements, securing sufficient dynamic storage, and initializing non-input variables.

I3DCMP. This routine resolves volume and junction block indices for the 3-D component.

CMP3DT. This is the common deck containing the 3-D component variables. The variables, and their definitions are:

nxcel1 Number of volumes in the $x$-direction.

nyce 11 Number of volumes in the $y$-direction.

nzce11 Number of volumes in the $y$-direction.

ncells Total number of volumes for the 3-D component.

njcel1 Total number of interior junctions for the 3-D component.

Calculated by:

$($ nxcel1 - 1)(nycel1)(nzcel1) +

$($ nxcel1) (nycell -1) (nzcel1) +

(nxce11)(nyce11)(nzcel1 -1)

nblsiz Storage requirements for volume oriented variables in the $3-D$ component.

nblskp Skip factor for the variables in above variables.

nb2siz Storage requirements for junction oriented variables in the 3-D component.

nblskp Skip factor for the above variables.

nxjuns Number of $x$-oriented junctions (nxce11 - 1)(nyce11)(nzcel1)

nyjuns Number of y-oriented junctions (nxce11)(nycel1 - 1) (nzcel1)

nzjuns Number of z-oriented junctions (nxce11)(nycel1)(nzce11 - 1)

cart3d Flag for rectangular or cylindrical coordinates.

offs3d Offset to 3-D component variables.

rzro3d Multiplier for rectangular or cylindrical coordinates.

rpm3d Multiplier for rectangular or cylindrical coordinates. 


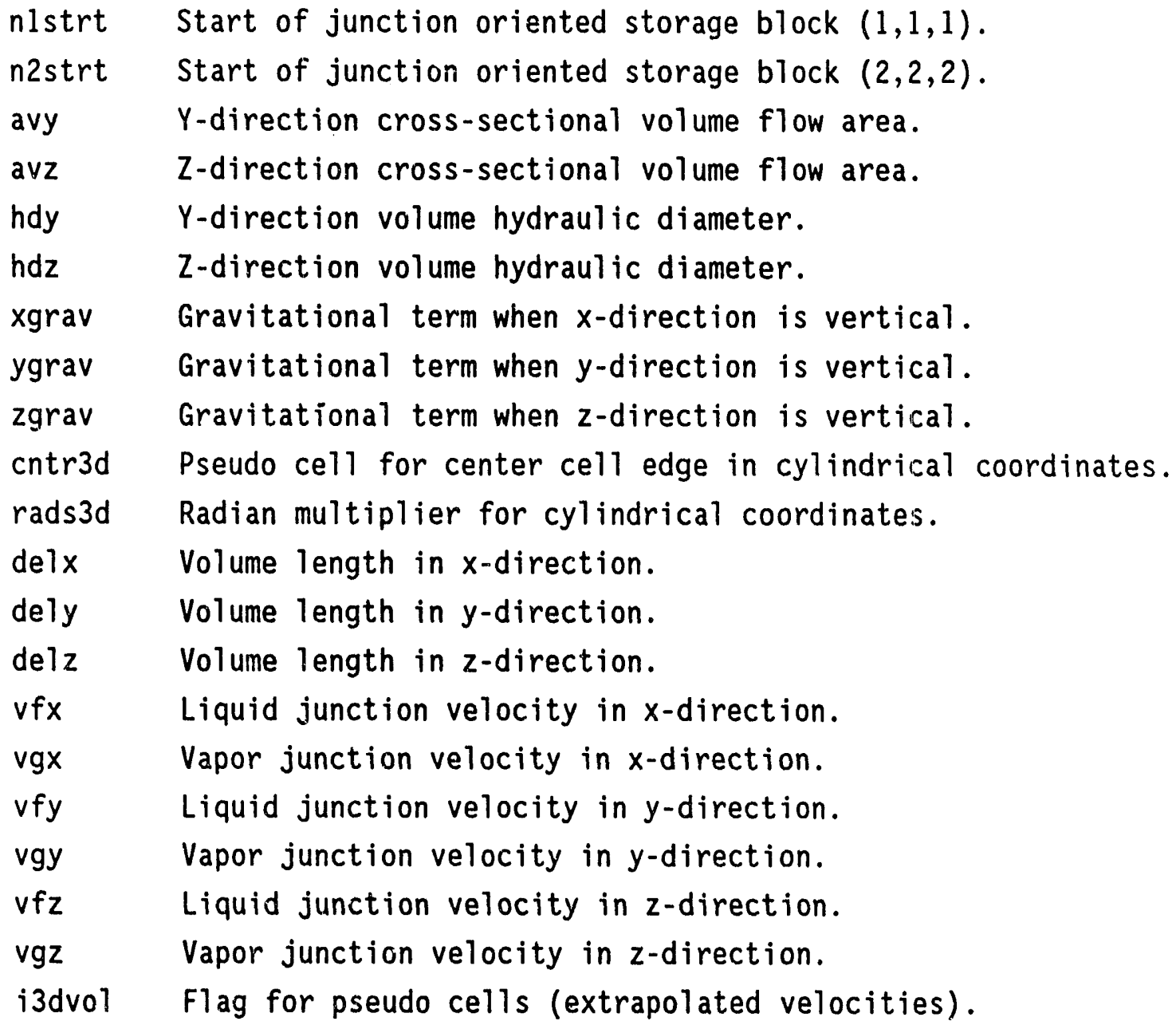

\subsection{References}

3-1. K. E. Carlson et al., Developmental Assessment of the Multidimensional Component in RELAP5 for Savannah River Site Thermal Hydraulic Analysis, EGC-EAST-9803, Rev. 0, July 1992.

3-2. K. E. Carlson, ATHENA Multidimensional Model, EGG Idaho, Inc., Draft Report, September 1987.

3-3. K. E. Carlson, P. A. Roth, and V. H. Ransom, ATHENA Codo Manual: EGG-RTH-7397, September 1986. 


\subsection{MULTIDIMENSIONAL CONSTITUTIVE MODELS}

\subsection{InTRODUCTION}

The following topics will be covered in this section: flow regimes, interphase friction, virtual mass, interphase heat transfer, wall friction, wall heat transfer regimes, and wall heat transfer. Since the 3-D model is being implemented in RELAP5/MOD2.5, which is primarily based on RELAP5/MOD2, one should consult both the RELAP5/MOD2 Code Manua $1^{4-1}$ and the RELAP5/MOD2 Models and Correlations document ${ }^{4-2}$ regarding the items presented in this section. The changes that were made to RELAP5/MOD2 in going to RELAP5/MOD2.5 can be found in the SCDAP/RELAP5/MOD2 Code Manual. 4-3 since the implementation of the 3-D model is a major change to the code, it is felt that the safest and least risky method is to first implement the 3-flow regime method. This method requires the least amount of coding, and hopefully will have minimal errors associated with it. After the integration and testing of the new 3-D input processing, volume velocities for each direction, additional momentum flux terms, 1-D to 3-D connections, and this 3-flow regime method, perhaps implementation of the 1 -flow regime method can begin.

\subsection{Flow Regimes}

The same volume flow maps (horizontal and vertical/annular) that are currently used in RELAP5/MOD2.5 for the normal 1-D volumes will also be used for 3-D volumes. These volume flow maps are shown in Figures 4-1 and 4-2. For the horizontal volume flow map, the volume flow regimes are bubbly (BBY), slug (SLG), annular mist (ANM), mist (MST), and horizontally stratified (HST). There are transitions between these regimes, if needed. For the vertical/annular volume flow map, the volume flow regimes are bubbly (BBY), slug (SLG), annular mist (ANM), mist (MST), inverted annular (IAN), inverted slug (ISL), and vertically stratified (VST). There are also transitions between these regimes, if needed. 

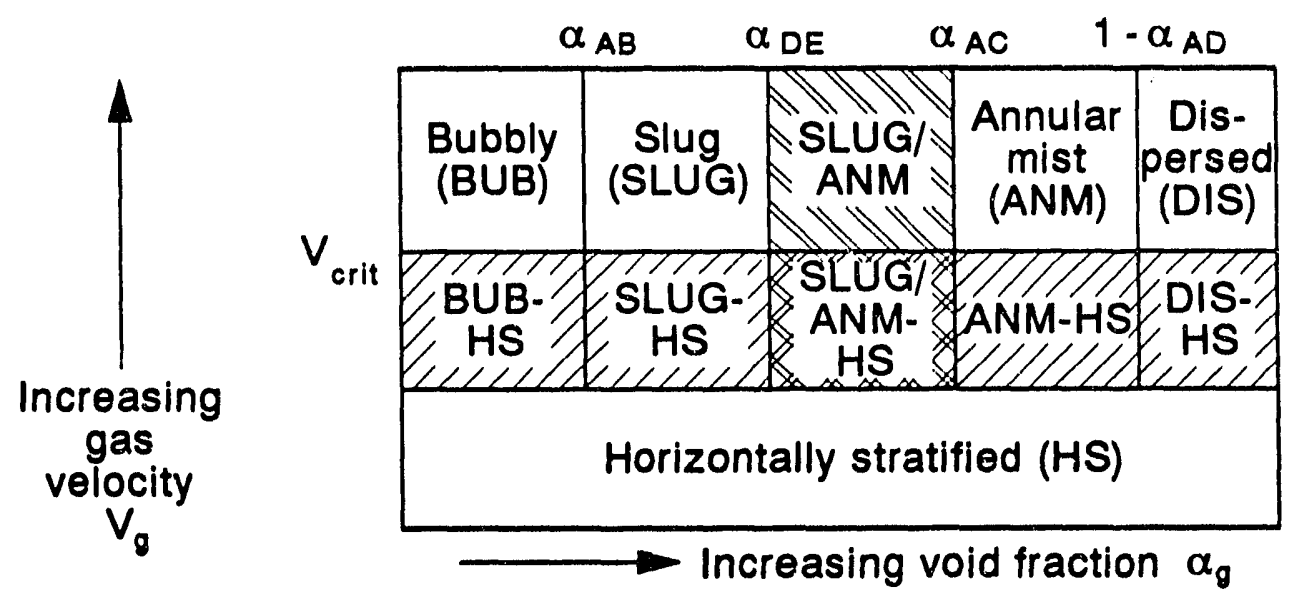

M552-WHT-492-30

Figure 4-1. Horizontal flow map and flow regimes. 


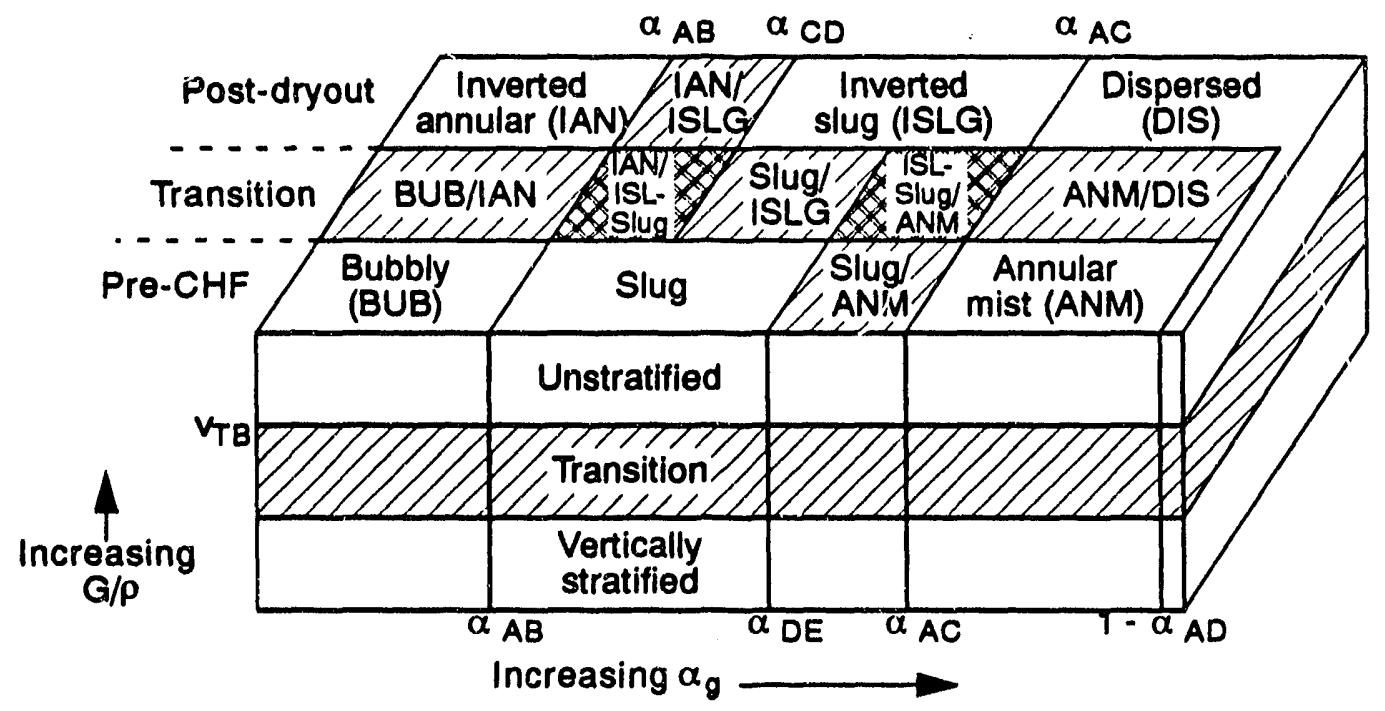

M552-WHT-492-31

Figure 4-2. Vertical/annular flow map and flow regimes. 
For the current constitutive models, the 3-flow regime method is used. In general, this means that for a 3-D volume, there are 3 directions, 3 volume flow maps, and 3 volume flow regimes. Thus, there will be a volume flow map and a volume flow regime associated with each direction. This is the general approach used in parts of the TRAC-B code $4-4,5$ and the THERMIT code. ${ }^{4-6}$ The notation used in this section is as follows: $\Delta z_{1}, \Delta z_{2}$, and $\Delta z_{3}$ are the elevations in the 3 directions; $\Delta x_{1}, \Delta x_{2}$, and $\Delta x_{3}$ are the lengths in the 3 directions; $v_{g 1}, v_{g 2}$, and $v_{g 3}$ are the vapor volume velocities in the 3 directions, $v_{f 1}, v_{f 2}$, and $v_{f 3}$ are the liquid volume velocities in the 3 directions, and $G_{1}, G_{2}$, and $G_{3}$ are the volume mass fluxes in the 3 directions. The terms $G_{1}, G_{2}$, and $G_{3}$ are given by:

$$
\begin{aligned}
& G_{1}=\left|\alpha_{g} \rho_{g} v_{g 1}+\alpha_{f} \rho_{f} v_{f 1}\right| \\
& G_{2}=\left|\alpha_{g} \rho_{g} v_{g 2}+\alpha_{f} \rho_{f} v_{f 2}\right| \\
& G_{3}=\left|\alpha_{g} \rho_{g} v_{g}+\alpha_{f} \rho_{f} v_{f 3}\right|
\end{aligned}
$$

For volume direction 1 , the direction 1 elevation angle $\phi_{1}$, is determined from

$$
\phi_{1}=\sin ^{-1}\left(\Delta z_{1} / \Delta x_{1}\right)
$$

For a horizontal map ( $\left|\phi_{1}\right| \leq 15$ degrees), $v_{g l}$ vs $\alpha_{g}$ is used to determine the flow regime (BBY, SLG, ANM, MST, HST) in direction 1. There is a mass flux criterion that uses $G_{1}$ to limit the slug flow regime. For a vertical/annular map $\left(\left|\phi_{1}\right|>15\right.$ degrees), $G_{1} / \rho=\left|\alpha_{g} \rho_{g} v_{g l}+\alpha_{f} \rho_{f} v_{f l}\right| / \rho$ vs $\alpha_{g}$ is used to determine the flow regime (BBY, SLG, ANM, MST, IAN, ISL, VST) in direction 1. As with the horizontal map in direction 1 , there is a mass flux criterion that uses $G_{1}$ to 1 imit the slug flow region.

For volume direction 2, the direction 2 elevation angle $\phi_{2}$ is determined from 


$$
\phi_{2}=\sin ^{-1}\left(\Delta z_{2} / \Delta x_{2}\right) .
$$

For a horizontal map ( $\left|\phi_{2}\right| \leq 15$ degrees), $v_{g 2}$ vs $\alpha_{g}$ is used to determine the flow regime (BBY, SLG, ANM, MST, HST) in direction 2. There is a mass flow criterion that uses $G_{2}$ to limit the slug flow region. For a vertical/annular map $\left(\left|\phi_{2}\right|>15\right.$ degrees $)$, $G_{2} / \rho=\left|\alpha_{g} \rho_{g} v_{g 2}+\alpha_{f} \rho_{f} v_{f 2}\right| / \rho$ vs $\alpha_{g}$ is used to determine the flow regime (BBY, SLG, ANM, MST, IAN, ISL, VST) in direction 2. As with the horizontal map in direction 2, there is a mass flux criterion that uses $G_{2}$ to 1 imit the slug flow region.

For volume direction 3 , the direction 3 elevation angle $\phi_{3}$ is determined from

$$
\phi_{3}=\sin ^{-1}\left(\Delta z_{3} / \Delta x_{3}\right)
$$

For a horizontal map ( $\left|\phi_{3}\right| \leq 15$ degrees), $v_{g 3}$ vs $\alpha_{g}$ is used to determine the flow regime (BBY, SLG, ANM, MST, HST) in direction 3. There is a mass flux criterion that uses $G_{3}$ to limit the slug flow region. For a vertical/annular map $\left(\left|\phi_{3}\right|>15\right.$ degrees), $G_{3} / \rho=\left|\alpha_{g} \rho_{g} v_{g 3}+\alpha_{f} \rho_{f} v_{f 3}\right| / \rho$ vs $\alpha_{g}$ is used to determine the flow regime (BBY, SLG, ANM, MST, IAN, ISL, VST) in direction 3. As with the horizontal map in direction 3 , there is a mass flux criterion that uses $G_{3}$ to 1 imit the slug flow region.

For future consideration for the constiultive models, the 1-flow regime method has been recommended by Rich Dimenna ${ }^{4-7}$ and John Trapp. 4-8 In general, this means that for a 3-D volume, there are 3 directions, 1 volume flow map, and 1 volume flow regime. This approach is more physically correct, in that when one takes a picture of the flow, there is only 1 flow regime. The 1-flow regime method is currently under consideration for includsion in the code. Additional notation needed in this section are as follows: $G$ is average volume mass flux, $v_{g}$ is the average vapor volume velocity, and $v_{f}$ is the average liquid volume velocity. To determine the volume flow map (either horizontal or vertical/annular), the following terms will need to be calculated: 


$$
\begin{aligned}
& G=\sqrt{G_{1}{ }^{2}+G_{2}{ }^{2}+G_{3}{ }^{2}} \\
& v_{g}=\sqrt{v_{g 1}{ }^{2}+v_{g 2}{ }^{2}+v_{g 3^{2}}} \\
& v_{f}=\sqrt{v_{f 1}{ }^{2}+v_{f 2}{ }^{2}+v_{f 3}{ }^{2}}
\end{aligned}
$$

Next, $G_{1}, G_{2}$, and $G_{3}$ will need to be compared to $G$ to find the dominate flow direction, and thus deciding if the flow map is horizontal or vertical. If the horizontal flow map is used, $v_{g} v s \alpha_{g}$ will then be used to determine the flow regime (BBY, SLG, ANM, MST, HST). If the vertical/annular flow is used, $G / \rho$ vs $\alpha_{g}$ will then be used to determine the flow regime (BBY, SLG, ANM, MST, IAN, ISL, VST).

\subsection{INTERPHASE FrICTION}

For the current model using the 3-flow regime method, three volume interphase drag coefficients are calculated. The correlations used are the same as those used in 1-D volumes. The volume interphase drag coefficient in direction 1 (FIVOL ${ }_{1}$ ) is calculated using direction 1 flow regime, $v_{f 1}, v_{g l}$, and $G_{1}$. The volume interphase drag coefficient in direction 2 ( $F I V O L_{2}$ ) is calculated using direction 2 flow regime, $v_{f 2}$, $v_{g 2}$, and $G_{2}$. The volume interphase drag coefficient in direction 3 $\left(\mathrm{FIVOL}_{3}\right.$ ) is calculated using direction 3 flow regime, $v_{f 3}, v_{g}$, and $G_{3}$. Next, at junctions between two 3-D volumes, volume-weighting similar to that used between two 1-D volumes are used. The notation used is as follows: $K 1$ is the index for the 'from' or $K$ volume in direction $1, L 1$ is the index for the 'to' or $L$ volume in direction $1, K 2$ is the index for the 'from' or $K$ volume in direction 2, $L 2$ is the index for the 'to' or $L$ volume in direction 2, $\mathrm{K} 3$ is the index for the 'from' or $K$ volunie in direction 3 , $L 3$ is the index for the 'to' or $L$ volume in direction 3 , and $V$ is the volume. The junction interphase drag coefficient in direction $1\left(F I J_{1}\right)$ is calculated from

$$
\mathrm{FIJ}_{1}=\frac{\mathrm{FIVOL}_{1}(\mathrm{~K} 1) \cdot V(K 1)+\mathrm{FIVOL}_{1}(\mathrm{~L} 1) \cdot \mathrm{V}(\mathrm{LI})}{V(K 1)+V(L 1)}
$$


The junction interphase drag coefficient in direction $2\left(F I J_{2}\right)$ is calculated from

$$
\mathrm{FIJ}_{2}=\frac{F I \mathrm{FOL}_{2}(K 2) \cdot V(K 2)+F I V O L_{2}(L 2) \cdot V(L 2)}{V(K 2)+V(L 2)}
$$

The junction interphase drag coefficient in direction $3\left(F I J_{3}\right)$ is calculated from

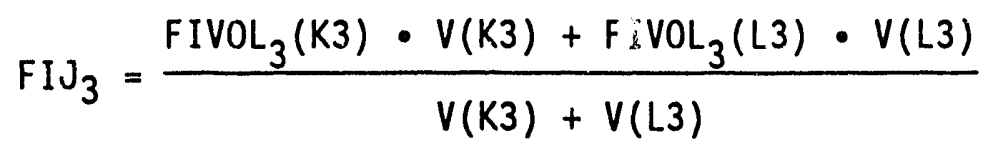

For future consideration using the 1 -flow regime method, only 1 volume interphase drag coefficient (FIVOL) will be calculated for each 3-D volume. It will be calculated using the volume flow regime, $v_{f}, v_{g}$, and $G$. The junction interphase drag coefficient in direction 1 (FIJ $)_{1}$ will be calculated from

$$
F I J_{1}=\frac{F I V O L(K 1) \cdot V(K 1)+F I V 0 L(L 1) \cdot V(L 1)}{V(K 1)+V(L 1)}
$$

The junction interphase drag coefficient in direction $2\left(F I J_{2}\right)$ wi1l be calculated from

$$
F I J_{2}=\frac{F I V O L(K 2) \cdot V(K 2)+F I V O L(L 2) \cdot V(L 2)}{V(K 2)+V(L 2)}
$$

The junction interphase drag coefficient in direction $3\left(\mathrm{FIJ}_{3}\right)$ will be calculated from

$$
F I J_{3}=\frac{F I V O L(K 3) \cdot V(K 3)+F I V O L(L 3) \cdot V(L 3)}{V(K 3)+V(L 3)}
$$




\subsection{Virtual Mass}

For the current model using the 3-flow regime method, three virtual mass coefficients are calculated. The volume virtual mass coefficient in direction $1\left(F A A V_{1}\right)$ is calculated using direction 1 flow regime, $v_{f l}, v_{g l}$, and $G_{1}$. The volume virtual mass coefficient in direction $2\left(F_{A A V}\right)$ is calculated using direction 2 flow regime, $v_{f 2}, v_{g 2}$, and $G_{2}$. The volume virtual mass coefficient in direction $3\left(F^{\prime} A V_{3}\right)$ is calculated using direction 3 flow regime, $v_{f 3}, v_{g 3}$, and $G_{3}$. Currently, for the 1-D case, the volume virtual mass coefficient depends only on $\alpha_{g}$, and this is also used for the 3-D case. Thus, FAAV $_{1}=$ FAAV $_{2}=$ FAAV $_{3}$. Recent RELAP5 calculations by Richards and Stopher ${ }^{4-9}$ for 1-D volumes indicate that the virtual mass coefficient should be set to 0 for stratified flow as suggested in the RELAP5 code manual. 4-1 This is not done in the present 1-D coding, and needs to be investigated for both $1-D$ and 3-D. Next, at junctions between two 3-D volumes, volume-weighting similar to that used between two 1-D volumes are used. The notation is the same as in the interphase drag. The junction virtual mass coefficient in direction $1\left(F^{\prime} A J_{1}\right)$ is calculated from

$$
\text { FAAJ }_{1}=\frac{\text { FAAV }_{1}(K 1) \cdot V(K 1)+F_{1 A V}(L 1) \cdot V(L 1)}{V(K 1)+V(L 1)}
$$

The junction virtual mass coefficient in direction $2\left(F A A J_{2}\right)$ is calculated from

$$
\mathrm{FAAJ}_{2}=\frac{\mathrm{FAAV}_{2}(K 2) \cdot V(K 2)+\mathrm{FAAV}_{2}(L 2) \cdot V(L 2)}{V(K 2)+V(L 2)}
$$

The junction virtual mass coefficient in direction $3\left(F^{\prime} A J_{3}\right)$ is calculated from

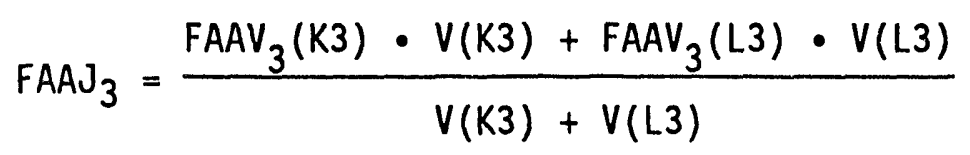


For future consideration using the 1 -flow regime method, only 1 volume virtual mass coefficient (FAAV) will be calculated for each 3-D volume. It will be calculated using the volume flow regime, $v_{f}, v_{g}$, and $G$. The junction virtual mass coefficient in direction 1 (FAAJ $)_{1}$ will be calculated from

$$
\text { FAAJ }_{1}=\frac{F A A V(K 1) \cdot V(K 1)+F A A V(L 1) \cdot V(L 1)}{V(K 1)+V(L 1)}
$$

The junction virtual mass coefficient in direction $2\left(\mathrm{FAAJ}_{2}\right)$ will be calculated from

$$
\text { FAAJ }_{2}=\frac{F A A V(K 2) \cdot V(K 2)+F A A V(L 2) \cdot V(L 2)}{V(K 2)+V(L 2)}
$$

The junction virtual mass coefficient in direction $3\left(\mathrm{FAAJ}_{3}\right)$ will be calculated from

$$
\mathrm{FAAJ}_{3}=\frac{\text { FAAV }(K 3) \cdot V(K 3)+\operatorname{FAAV}(L 3) \cdot V(L 3)}{V(K 3)+V(L 3)}
$$

\subsection{Interphase Heat Transfer}

For the current model using the 3-flow regime method, three sets of volume interphase heat transfer coefficients are calculated. The same correlations used in the 1-D case are used in the 3-D case. The volume interphase heat transfer coefficients in direction 1 ( $H I F_{1}$ and $\left.H I G_{1}\right)$ are calculated using direction 1 flow regime, $v_{f l}, v_{g l}$, and $G_{1}$. The volume interphase heat transfer coefficients in direction $2\left(\mathrm{HIF}_{2}\right.$ and $\left.\mathrm{HIG}_{2}\right)$ are calculated using direction 2 flow regime, $v_{f 2}, v_{g 2}$, and $G_{2}$. The volume interphase heat transfer coefficients in direction $3\left(\mathrm{HIF}_{3}\right.$ and $\left.\mathrm{HIG}_{3}\right)$ are calculated using direction 3 flow regime, $v_{f 3}, v_{g 3}$, and $G_{3}$. Since only one volume liquid interphase heat transfer coefficient (HIF) and one volume vapor interphase heat transfer coefficient (HIG) are needed, some averaging is used. The notation used is as follows: $D_{1}, D_{2}$, and $D_{3}$ are the volume hydraulic diameters in the 3 directions. The formulas used are 


$$
\begin{aligned}
& H I F=\frac{H I F_{1} \cdot D_{1}+H I F_{2} \cdot D_{2}+H I F_{3} \cdot D_{3}}{D_{1}+D_{2}+D_{3}} \\
& H I G=\frac{H I G_{1} \cdot D_{1}+H I G_{2} \cdot D_{2}+H I G_{3} \cdot D_{3}}{D_{1}+D_{2}+D_{3}}
\end{aligned}
$$

If the flow regime is vertically stratified (VST) in one of the directions, the HIF is set to the HIF from the vertically stratified direction, and HIG is set to the HIG from the vertically stratified direction.

For future consideration using the 1-flow regime method, only 1 volume liquid interphase heat transfer coefficient (HIF) and 1 volume vapor interphase heat transfer coefficient (HIG) will be calculated for each 3-D volume. They will be calculated using the volume flow regime, $v_{f}, v_{g}$, and $G$.

\subsection{WALL FRICTION}

For the current model using the 3-flow regime method, three sets of volume wall void fractions are calculated. The same formulas used in the 1-D case are also used in the 3-D case. The volume wall void fractions in direction $1\left(\alpha_{\mathrm{fWl}}\right.$ and $\left.\alpha_{\mathrm{gwl}}\right)$ are calculated using direction 1 flow regime, $v_{f 1}, v_{g l}$, and $G_{1}$. The volume wall void fractions in direction 2 $\left(\alpha_{f w 2}\right.$ and $\left.\alpha_{g w 2}\right)$ are calculated using direction 2 flow regime, $v_{f 2}$, $v_{\mathrm{g} 2}$, and $G_{2}$. The volume wall void fractions in direction $3\left(\alpha_{\mathrm{fw}}\right.$ and $\left.\alpha_{g_{3} 3}\right)$ are calculated using direction 3 flow regime, $v_{f 3}, v_{g 3}$, and $G_{3}$. The same wall friction correlations used in the 1-D case are used in the 3-D case. The volume wall friction coefficients in direction 1 (FWFVOL 1 and FWGVOL $_{1}$ ) are calculated using direction 1 wall void fractions, $v_{f l}$, $v_{g l}$, and $G_{1}$. The volume wall friction coefficients in direction 2 (FWFVOL 2 and FWGVOL $_{2}$ ) are calculated using direction 2 wall void fractions, $v_{f 2}, v_{g 2}$, and $G_{2}$. The volume wall friction coefficients in direction 3 ( $\mathrm{FWFVOL}_{3}$ and $\mathrm{FWGVOL}_{3}$ ) are calculated using direction 3 wall void fractions, $v_{f 3}, v_{g}$, and $G_{3}$. Next, at junctions between two 3-D 
volumes, length-weighting similar to that used between two 1-D volumes will be used. The notation is the same as in the interphase drag. The junction wall friction coefficients in direction 1 (FWFJ 1 and $\left.F W J_{1}\right)$ are calculated from

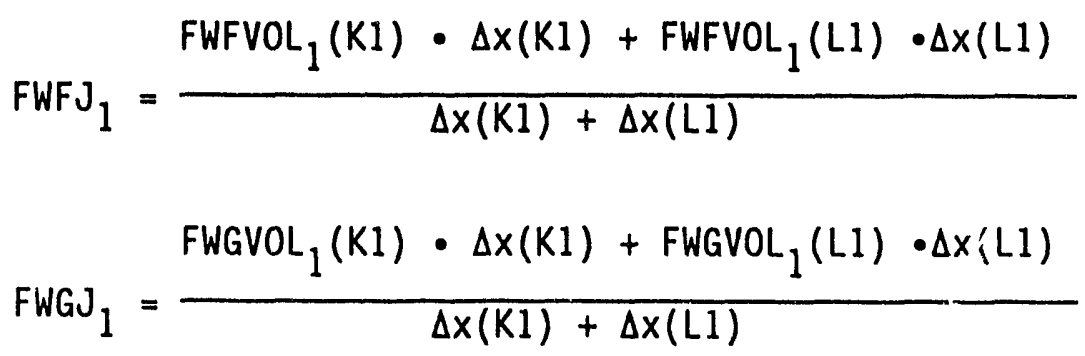

The junction wall friction coefficients in direction $2\left(\mathrm{FWFJ}_{2}\right.$ and $\mathrm{FWGJ}_{2}$ ) are calculated from

$\mathrm{FWFJ}_{2}=\frac{\mathrm{FWFVOL}_{2}(\mathrm{~K} 2) \cdot \Delta x(K 2)+\mathrm{FWFVOL}_{2}(\mathrm{~L} 2) \cdot \Delta x(\mathrm{~L} 2)}{\Delta x(\mathrm{~K} 2)+\Delta x(\mathrm{~L} 2)}$
$\mathrm{FWGJ}_{2}=\frac{\mathrm{FWGVOL}_{2}(\mathrm{~K} 2) \cdot \Delta x(K 2)+\mathrm{FWGVO}_{2}(\mathrm{~L} 2) \cdot \Delta x(\mathrm{~L} 2)}{\Delta x(\mathrm{~K} 2)+\Delta x(\mathrm{~L} 2)}$

The junction wali friction coefficients in direction $3\left(\mathrm{FWFJ}_{3}\right.$ and $\mathrm{FWGJ}_{3}$ ) are calculated from

$$
\begin{aligned}
& \mathrm{FWFJ}_{3}=\frac{\mathrm{FWFVOL}_{3}(\mathrm{~K} 3) \cdot \Delta x(K 3)+\mathrm{FWFVOL}_{3}(\mathrm{~L} 3) \cdot \Delta x(\mathrm{L3})}{\Delta x(K 3)+\Delta x(\mathrm{L3})} \\
& \mathrm{FWGJ}_{3}=\frac{\mathrm{FWGVL}_{3}(\mathrm{~K} 3) \cdot \Delta x(K 3)+\mathrm{FWGVOL}_{3}(\mathrm{~L} 3) \cdot \Delta x(\mathrm{~L} 3)}{\Delta x(\mathrm{~K} 3)+\Delta x(\mathrm{~L} 3)}
\end{aligned}
$$

For future consideration using the 1-flow regime method, only 1 volume liquid wall void fraction $\left(\alpha_{f w}\right)$ and 1 volume vapor wall void fraction $\left(\alpha_{g w}\right)$ will be calculated for each 3-D volume. They will be calculated using the volume flow regime, $v_{f}, v_{g}$, and $G$. For the volume wall frictions, the current thinking is to allow different correlations in different directions. This is what is done in THERMIT. 4-6 The volume 
wall friction coefficients in direction 1 (FWFVOL 1 and FWGVOL $_{1}$ ) will be calculated using wall void fractions, $v_{f 1}, v_{g l}$, and $G_{1}$. The volume wall friction coefficients in direction 2 (FWFVOL 2 and FWGVOL $_{2}$ ) will be calculated using wall void fractions, $v_{f 2}, v_{g 2}$, and $G_{2}$. The volume wall friction coefficients in direction 3 (FWFVOL 3 and $\mathrm{FWGVOL}_{3}$ ) will be calculated using wall void fractions, $v_{f 3}, v_{g 3}$, and $G_{3}$. The junction wall friction coefficients in direction 1 (FWFJ 1 and $F W G_{1}$ ) will be calculated from

$$
\begin{aligned}
& \mathrm{FWFJ}_{1}=\frac{\mathrm{FWFVOL}_{1}(\mathrm{~K} 1) \cdot \Delta x(K 1)+\mathrm{FWFVOL}_{1}(\mathrm{~L} 1) \cdot \Delta x(\mathrm{~L} 1)}{\Delta x(\mathrm{~K} 1)+\Delta x(\mathrm{LI})} \\
& \text { FWGVOL }_{1}(K 1) \cdot \Delta x(K 1)+F W G V O L_{1}(L 1) \cdot \Delta x(L 1) \\
& \mathrm{FWGJ}_{1}=\frac{1}{\Delta x(K 1)+\Delta x(L 1)}
\end{aligned}
$$

The junction wall friction coefficients in direction $2\left(F^{2} F_{2}\right.$ and $\mathrm{FWGJ}_{2}$ ) will be calculated from

$$
\begin{aligned}
& \mathrm{FWFJ}_{2}=\frac{\mathrm{FWFVOL}_{2}(\mathrm{~K} 2) \cdot \Delta x(K 2)+\mathrm{FWFVOL}_{2}(\mathrm{LL}) \cdot \Delta x(L 2)}{\Delta x(K 2)+\Delta x(L 2)} \\
& \mathrm{FWGJ}_{2}=\frac{\mathrm{FWGOL}_{2}(\mathrm{~K} 2) \cdot \Delta x(K 2)+\mathrm{FWGVOL}_{2}(\mathrm{~L} 2) \cdot \Delta x(L 2)}{\Delta x(K 2)+\Delta x(L 2)}
\end{aligned}
$$

The junction wall friction coefficients in direction $3\left(\mathrm{FWFJ}_{3}\right.$ and $\mathrm{FWGJ}_{3}$ ) will be calculated from

$$
\begin{aligned}
& \mathrm{FWFJ}_{3}=\frac{\mathrm{FWFOL}_{3}(\mathrm{~K} 3) \cdot \Delta x(K 3)+\mathrm{FWFVOL}_{3}(\mathrm{~L} 3) \cdot \Delta x(\mathrm{~L} 3)}{\Delta x(\mathrm{~K} 3)+\Delta x(\mathrm{~L} 3)} \\
& \mathrm{FWGJ}_{3}=\frac{\mathrm{FWGOL}_{3}(\mathrm{~K} 3) \cdot \Delta x(\mathrm{~K} 3)+\mathrm{FWGVO}_{3}(\mathrm{~L} 3) \cdot \Delta x(\mathrm{~L} 3)}{\Delta x(\mathrm{~K} 3)+\Delta x(L 3)}
\end{aligned}
$$




\subsection{Wall heat transfer Regimes}

The same wall heat transfer regimes as used in the 1-D case (see Figure 4-3) are used in the 3-D case. The wall temperature $\left(T_{w}\right)$, saturation temperature $\left(T_{s a t}\right)$, and vapor void fraction $\left(\alpha_{g}\right)$ are the primary variables used in the decision logic, and they are all independent of direction.

\section{8 Wall Heat transfer}

For the current model using the 3-flow regime method, the same heat transfer correlations used in the 1-D are used. If the flow regime in the adjacent volume is vertically stratified in one of the directions, then the heat transfer coefficients are modified as is done in the 1-D case.

For future consideration using the 1-flow regime method, the same heat transfer correlations used in the 1-D case will be used. If the flow regime in the adjacent volume is vertically stratified, then the heat transfer coefficients will be modified as is done in the 1-D case.

\subsection{References}

4-1. V. H. Ransom, et a1., RELAP5/MOD2 Code Manual, Volume 1, NUREG/CR-4312, EGG-2396, Rev. 1, March 1987.

4-2. R. A. Dimenna, et a1., RELAP5/MOD2 Models and Correlations, NUREG/CR-5194, EGG-2531, August 1988.

4-3. C. M. Allison and E. C. Johnson (Eds), SCDAP/RELAP5/MOD2 Code Manual, Volume 1, NUREG/CR-5273, EGG-2555, September 1989.

4-4. W. L. Weaver, et al., TRAC-BFI Manual: Extensions to TRAC-BD1/MOD1, NUREG/CR-4391, EGG-2417, August 1986.

4-5. S. Z. Rouhani, et al., TRAC-BF1 Models and Correlations, EG\&G Idaho, Inc., Draft Report, February 1988.

4-6. J. Loomis, et a1., THERMIT: A Computer Program for Three-Dimensional Therma7-Hydraulic Analysis of Light Water Reactor Cores, EPRI NP-2032, September 1981.

4-7. R. A. Dimenna, Private Communication, Westinghouse Savannah River, November 1990. 


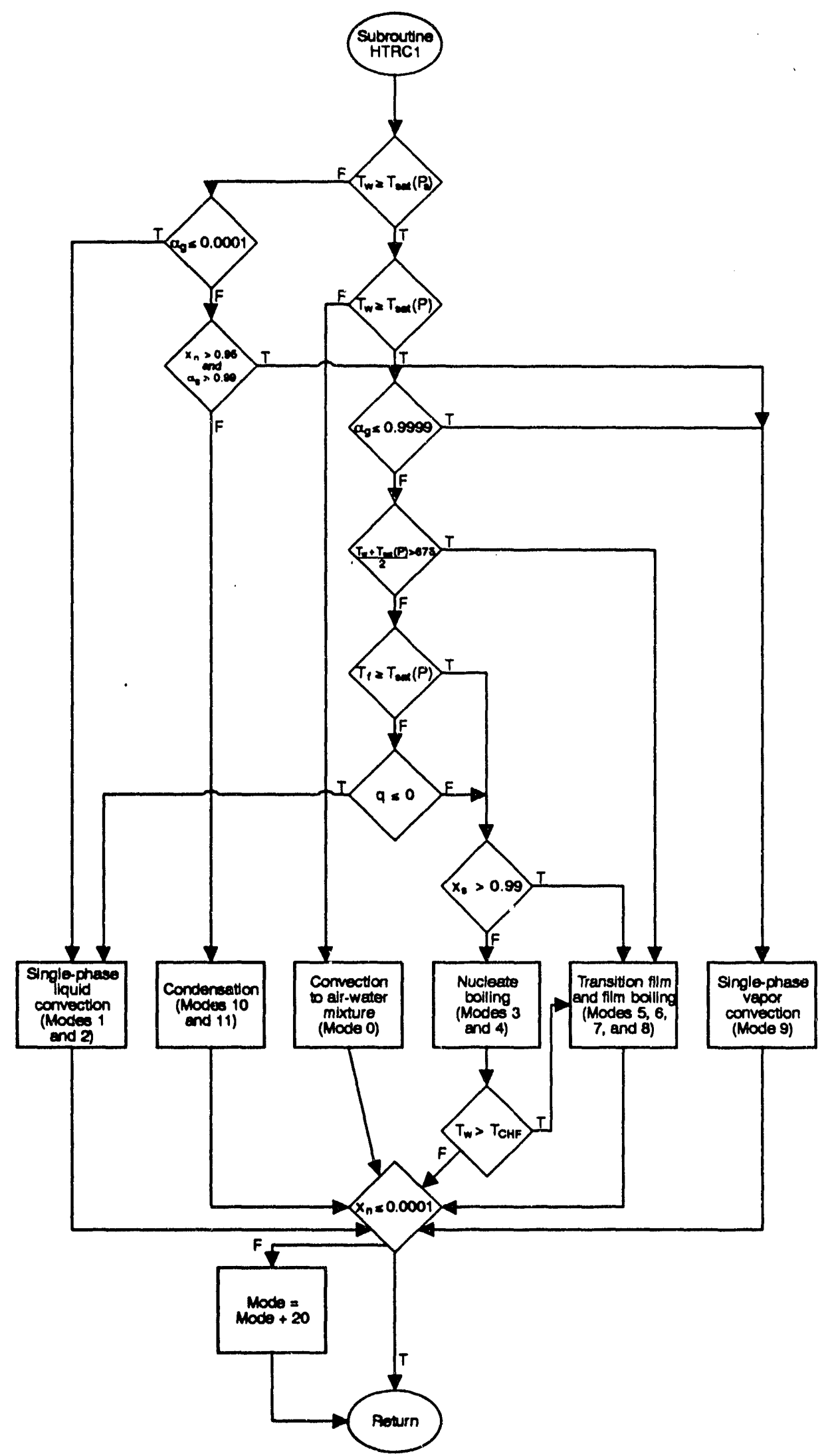

M552-WHT-492-32

Figure 4-3. Wall heat transfer regimes. 
4-8. J. A. Trapp, Private Communication, University of Colorado, November 1990.

4-9. C. G. Richards and I. S. Stopher, Stratified Flow Benchmark Calculations Using RELAP5/MOD2, 1990 Joint RELAP5 and TRAC-BWR International User Seminar, September 17-20, 1990, Chicago, Ilinois. 


\subsection{MULTIDIMENSIONAL INPUT REQUIREMENTS}

A multidimensional component is indicated by MULTID on Card CCCO000. This component defines a one, two, or three dimensional array of volumes and the internal junctions connecting the volumes. The multidimensional component is described as a three dimensional component but can be reduced to two or one dimensions by defining only one interval in the appropriate coordinate directions. The geometry can be either Cartesian $(x, y, z)$ or cylindrical $(r, \theta, z)$. In cylindrical geometry, the $r$ direction can start at zero or nonzero, and $\theta$ can cover 360 degrees (i.e., a full circle) or can cover less than 360 degrees (wedge shape, semicircle, etc).

An orthogonal, three dimensional grid is defined by mesh interval input data in each of the tihree coordinate directions. The edges of the hydrodynamic volumes are defined by the grid lines. Given NX intervals in the $x$ or $r$ coordinate direction, NY intervals in the $y$ or $\theta$ coordinate direction, and $N Z$ intervals in the $z$ coordinate direction, $N X \star N Y \star N Z$ volumes are defined. The number of volumes in a three dimensional component is limited to 999 volumes. Volumes are numbered CCCXYYZZO where $X, Y Y$, and $Z Z$ are the position numbers in the three coordinate directions. Position numbers in each coordinate direction start with one at the origin and increase consecutively in the positiva coordinate direction. $X$ represents the position number for the first coordinate direction which is $x$ in Cartesian geometry and $r$ in cylindrical geometry. The use of one digit for the first coordinate limits the number of volumes in that coordinate to nine volumes. YY represents the position number of the second coordinate direction which is $y$ in Cartesian geometry and $\theta$ in cylindrical geometry. The positive $\theta$ direction is in the counter clockwise direction. $Z Z$ represents the position number of the third coordinate which is the $z$ coordinate direction in both geometries. The use of two digits for the second and third directions allows up to 99 volumes in those coordinate directions. The maximum values of $X, Y Y$, and $Z Z$ are $N X, N Y$, and $N Z$ respectively.

The volume face number is given by CCCXYYZZF where the face number, $F$, is added to the volume number. The face numbers are 1 and 2 for the inlet and outlet faces respectively of the $x$ or $r$ coordinate, 3 and 4 for the 
inlet and outlet faces for the $y$ or $\theta$ coordinate, and 5 and 6 for the inlet and outlet faces for the $z$ coordinate. The volume-face number is the volume connection code used in the "from" and "to" portion of junction input.

Junctions for this component are generated between all internal faces, that is all faces common to the volumes in the component. The number of junctions is $(N X-1) * N Y * N Z+N X *(N Y-1) * N Z+N X * N Y *(N Z-1)$ for Cartesian geometry and for cylindrical geometry where the $\theta$ coordinate does not cover a full circle. When the $\theta$ coordinate covers 360 degrees, the number of junctions increases by $N X * N Z$. The coordinate directions of the junctions are aligned in the positive directions of the coordinates. For numbering purposes, the junctions are associated with the "from" face of the two volumes being joined. Thus, the junctions are numbered CCCXYYZZF where $F$ is limited to 2,4 , or 6 .

External junctions may connect to any exterior faces of the volumes and also any interior faces. External junctions connecting to internal faces imply branching or merging flow since internal junctions connect all internal faces. Some adjustments to the volumes and flow areas of the volumes and flow areas of the junctions should be made to account for the piping necessary to reach the internal face.

\subsection{CaRd CCCO001, MULTID Information Card}

This card is required.

WI(I) NUMBER OF $X$ OR R COORDINATE INTERVALS (NX). This word must be greater than zero and less than 10.

W2(I) NUMBER OF $Y$ OR $\theta$ COORDINATE INTERVALS (NY). This word must be greater than zero and less than 100.

W3(I) NUMBER OF $Z$ COORDINATE INTERVALS (NZ). This word must be greater than zero and less than 100. 
W4(I) VELOCITY/MASS FLOW FLAG. This word is optional and if missing, is assumed to be zero. The initial junction conditions are velocities if zero is entered and are mass flows if one is entered.

W5(I) GEOMETRY AND $\theta$ FLAG. This word is optional and if missing, is assumed to be zero. If this word is zero, Cartesian geometry is indicated, and if the magnitude of this word is one, cylindrical geometry is indicated. If the sign of this word is positive, $\theta$ is assumed to extend to 360 degrees; if the sign is negative, $\theta$ is assumed to extend to less than 360 degrees. The extra plane of junctions perpendicular to the $\theta$ direction exists when this quantity is one and does not exist when this quantity is zero or minus one. The sign is appropriate only to cylindrical geometry.

W6(R) VALUE OF INNERMOST RADIAL COORDINATE ( $m, f t)$. This word is optional and if missing is assumed 0.0. This word must be zero in Cartesian geometry, and must be greater than or equal to zero in cylindrical geometry. A nonzero value allows the specification of a cylinder with a hollow center.

W7(I) THREE DIMENSIONAL FLAG. This word is optional and if missing is assumed to be zero. If this word is zero, the three dimensional momentum equations are used. If this word is one, the normal one dimensional momentum equations are used on each of the coordinate directions. Cross flow indicators may not be used if this word is zero but may be used if this word is one.

\subsection{Card CCC0002, Rotation Angle Data Card}

This card is optional and if missing, the following angles are assumed to be zero.

W1(R) HORIZONTAL ROTATION ANGLE (degrees). 
W3(R) AZIMUTHAL ROTATION ANGLE (degrees).

The names of the angles above are not very accurate but are used here to relate to the angles used in other components such as a pipe. In a pipe or single volume, the horizontal angle specified the rotation of a volume in the horizontal plane, the vertical angle specified the rotation of a volume in the vertical plane, and the azimuthal angle was not used. The horjzontal angle had to be entered, but other than being checked for its absolute value being less than or equal to 360 degrees, was not used. The vertical angle, whose absolute angle had to be equal to or less than 90 degrees, was checked and was used to specify the direction of gravity.

The angles used in MULTID are more accurately known as Euler angles which are commonly used to describe the position of a rigid body in space. The reference coordinates are the standard right hand $x, y, z$ coordinate system in Cartesian geometry. The $z$ axes of both Cartesian and cylindrical geometries coincide and are oriented in the vertical direction with the positive direction being upward. The $x, y$ and $r, \theta$ coordinates are in the horizontal plane with the $\theta=0$ line coinciding with the $x$ axis. The coordinate system of the multidimensional component is initially aligned with the reference coordinates. The first angle specifies the rotation of the component's coordinate system about its original $z$ axis, the second angle is the rotation of the component's coordinate system about its new $x$ axis, and the third angle is the rotation of the component's coordinate system about its new $z$ axis. A positive angle specifies counterclockwise rotation as viewed by an observer on the positive part of the rotation axis looking towards the origin. In a 90 degree counterclockwise rotation about the $z$ axis, the position of the $x$ axis would be moved to the previous position of the $y$ axis. Similarly, a 90 degree counterclockwise rotation about the $x$ axis would move the $y$ axis to the previous position of the $z$ axis. The input range of each Euler angle is from 0.0 through plus or minus 360 degrees. 
The effects of the horizontal and vertical angles of the pipe volumes and the Euler angles are similar within the limitations of not having an azimuthal angle and the vertical angle being limited to 90 degrees.

The most common usage of the Euler angle data is to move the axial coordinate in cylindrical geometry from the vertical direction to the horizontal plane. The following input accomplishes that: 0.090 .00 .0 . If the azimuthal noding consisted of six 60 degree mesh intervals, two of the azimuthal grid lines would coincide with the vertical direction. If the third angle was specified as plus or minus 30 degrees, the center of two of the azimuthal intervals would lie on a vertical line.

The Euler angle data has not been completely implemented for cylindrical geometry and the vertical rotation angle (Word 2) must be 0.0 or 180.0 . The rotation capability was not a requirement for the initial multidimensional capability, and, as a result, it was not fully implemented in the other portions of the code. It is anticipated this will become fully operational in the future.

\subsection{Cards cccoxnn, Mesh Interval Cards}

These required cards enter the mesh interval data for the three coordinate directions. The digit $x$ is 1 for the $x$ or $r$ coordinate, 2 for the $y$ or $\theta$ coordinate, and 3 for the $z$ coordinate. The NN digits which may range from 01 through 99 sequences the cards within a series and need not be consecutive. One or more sets of data in sequential expansion format may be entered on each card.

WI (R) MESH INTERVAL ( $m, f t$, or degrees).

W2(I) VOLUME COORDINATE NUMBER. The number of the first volume coordinate number next to the origin is 1 and the last volume number is NX for $X$-coordinate, NY for $y$ coordinate, or NZ for $z$ coordinate.

The last volume coordinate number must equal NX, NY, or NZ depending on the value of $x$. 
In cylindrical geometry, the $\theta$ coordinates are entered in degrees. The sum of the intervals cannot exceed 360 degrees. The sum must equal 360 degrees if $W 5$ on Card CCCOOOl is 1 and must not equal 360 degrees if W5 is -1 .

Presently a further restriction exists on the $\theta$ intervals when the innermost radius is zero. For this case, the number of $\theta$ intervals must be even and the intervals must have 180 degree symmetry.

\subsection{Cards cccinnn, Volume Option Cards}

These cards are optional. NNN ranges from 001 through 999 and need not be consecutive.

These cards use an overlay format. The first six words define a rectangular solid in Cartesian geometry and an annular segment in cylindrical geometry. Tre solid figure consists of volumes where the $x$ or $r$ coordinate number ranges from $X 1$ through $X 2$, the $y$ or $\theta$ coordinate number ranges from $Y 1$ through $Y 2$, and the $z$ coordinate number ranges from $Z 1$ through $\mathrm{Z2}$.

The quantities following the six overlay numbers apply to the jolumes within the solid defined by the overlay. Overlays may reference any volume more than once; the data used in a volume is the information in the last overlay referencing that volume. Default data is stored in volumes not referenced by an overlay.

$\begin{array}{ll}\text { W1(I) } & \text { X1 } \\ \text { W2(I) } & \text { X2 } \\ \text { W3(I) } & \text { Y1 } \\ \text { W4(I) } & \text { Y2 } \\ \text { W5(I) } & \text { Z1 }\end{array}$


W6(I) 22

W7 $(R)$ VOLUME FACTOR. Default is 1.0 . This quantity must be greater than zero and less than or equal to one. The original volume of each volume is computed from the mesh interval data. The actual volumes's volume may be reduced by the factor in this word to account for solid material such as fuel pins within the volume. It could also account for piping which accesses interior volumes. The volume flow areas for the three coordinate directions are computed from the actual volume divided by the volume's length along the coordinate direction. The length is the appropriate mesh interval except for the $\theta$ direction where it is the mesh interval times the radius to the midpoint of the volume.

W8(I) SCALER VOLUME FLAG. This word applies to the scaler oriented options for a volume.

W9(I) X OR R COORDINATE VOLUME FLAG.

W10(I) Y OR $\theta$ COORDINATE VOLUME FLAG.

W11(I) $Z$ COORDINATE VOLUME FLAG.

W8 through w11 use a packed format pvbfe. Only the e option may be used for the scaler volume flag (W8), and the e option may not be used for the coordinate flags. The default values for the flags are 00000 .

The digit $\mathrm{p}$ specifies whether the water packing scheme is to be used. $\mathrm{p}=0$ specifies that the water packing scheme is to be used for the volume, and $\mathrm{p}=1$ specifies that the water packing scheme is not to be used.

The digit $\underline{v}$ specifies whether the vertical stratification model is to be used. $\underline{v}=0$ specifies that the vertical stratification model is to be used, and $\underline{v}=1$ specifies that the vertical stratification model is not to be used. 
The digit $\underline{b}$ specifies the interphase friction to be used. $\underline{b}=1$ means that the Bestion/Analytis rod bundle interphase friction model is to be applied; $\underline{b}=0$ means that the normal interphase friction model is to be applied.

The digit $f$ specifies whether wall friction is to be computed. $f=0$ specifies that wall friction effects are to be computed for the volume, and $f=1$ specifies that wall friction effects are not to be computed.

The digit e specifies if nonequilibrium or equilibrium is to be used. $\underline{e}=0$ specifies that a nonequilibrium (unequal temperature) calculation is to be used, and e=l specifies that an equilibrium (equal temperature) calculation is to be used. Equilibrium volumes should not be connected to nonequilibrium volumes. The equilibrium option is provided only for comparison to other codes.

\subsection{Cards cCc2nnN, Volume friction Data Cards}

These cards are optional. NNN ranges from 001 through 999 and need not be consecutive.

These cards use an overlay format similar to the CCCINNN cards described above. Not all volumes need to be referenced by the overlay.

$\begin{array}{ll}\text { W1(I) } & \text { XI } \\ \text { W2(I) } & \text { X2 } \\ \text { W3(I) } & \text { Y1 } \\ \text { W4(I) } & \text { Y2 } \\ \text { W5(I) } & \text { Z1 } \\ \text { W6(I) } & \text { Z2 }\end{array}$


W7(R) WALL ROUGHNESS FOR THE $X$ OR R COORDINATE $(m, f t)$. Default value is zero.

W8(R) HYDRAULIC DIAMETER FOR THE $X$ OR $R$ COORDINATE $(m, f t)$. Default value is zero. If zero, the hydraulic diameter is computed from four times the flow area divided by the wetted perimeter. The flow area and wetted perimeter are appropriate for the geometry and the coordinate direction.

W9(R) WALL ROUGHNESS FOR THE Y OR $\theta$ COORDINATE $(\mathrm{m}, \mathrm{ft})$. Defautt value is zero.

W10(R) HYDRAULIC DIAMETER FOR THE $Y$ OR $\theta$ COORDINATE $(\mathrm{m}, \mathrm{ft})$. Default value is zero. If zero, the hydraulic diameter is computed from four times the flow area divided by the wetted perimeter. The flow area and wetted perimeter are appropriate for the geometry and the coordinate direction.

W11(R) WALL ROUGHNESS FOR THE $z$ COORDINATE $(m, f t)$. Default value is zero.

W12(R) HYDRAULIC DIAMETER FOR THE $z$ COORDINATE $(m, f t)$. Default value is zero. If zero, the hydraulic diameter is computed from four times the flow area divided by the wetted perimeter. The flow area and wetted perimeter are appropriate for the geometry and the coordinate direction.

\subsection{Cards CCC3001 Through CCC5999, Junction Data Cards}

These cards are optional. The range of card numbers need not be consecutive.

These cards use the first six words as overlay information to specify a range of volumes and in addition use a seventh number to specify the volume face. The information following the face number is applied to the junction originating from the specified face of each volume included in the overlay. Not all junctions need to be referenced by these cards. 


$\begin{array}{ll}\text { W1(I) } & \text { X1 } \\ \text { W2(I) } & \text { X2 } \\ \text { W3(I) } & \text { Y1 } \\ \text { W4(I) } & \text { Y2 } \\ \text { W5(I) } & \text { Z1 } \\ \text { W6(I) } & \text { Z2 }\end{array}$

W7(I) FACE NUMBER. The face number is 1 imited to 2 , 4 , or 6 .

W8(R) JUNCTION AREA FACTOR. Default value is one. This quantity must be greater than zero and less than or equal to one. The original junction area is computed from the mesh interval data. The actual area is the original area times this factor. This quantity can be used to account for solid structures within the volume.

WY $(R)$ FORWARD FLOW ENERGY LOSS COEFFICIENT. Default value is zero.

WIO(R) REVERSE FLOW ENERGY LOSS COEFFICIENT. Default value is zero.

W11(I) JUNCTION CONTROL FLAGS. This word has the packed format fvcahs. The default value is 000000 .

The digit $f$ specifies CCFL options; $f=0$ means that the CCFL mode 1 is not applied, and $f=1$ means that it is applied.

The digit $\underline{v}$ specifies horizontal stratification entrainment/ pullthrough options; $\underline{v}=0$ means a centrally (side) located junction; $\underline{v}=1$ means an upward-oriented junction; $\underline{v}=2$ means a downward-oriented junction; and $\underline{v}=3$ means the model is not applied. 
The digit $\underline{c}$ specifies choking options. $\underline{c}=0$ means that the choking model is applied, and $c=1$ means that the choking model is not applied.

The digit a specifies area change options. $\underline{a}=0$ means either a smooth area change or no area change, and $\underline{a}=1$ means an abrupt area change.

The digit $\underline{h}$ specifies nonhomogeneous or homogeneous; $\underline{h}=0$ specifies the nonhomogeneous (two-velocity momentum equations) option, and $\underline{h}=2$ specifies the homogeneous (single-velocity momentum equation) option. For the homogeneous $(\underline{h}=2)$, the major edit printout will show a 1 .

The digit $\underline{s}$ specifies normal or crossflow junction. $\underline{s}=0$ specifies a normal junction. $\underline{s}=1$ specifies a crossflow junction and that the to volume is a crossflow volume. $\underline{s}=2$ specifies a crossflow junction and that the from volume is a crossflow volume. $\underline{s}=3$ specifies a crossflow junction and that both the from and to volumes are crossflow volumes. If a crossflow junction is specified, it is recommended that loss coefficients (other than 0.0 ) be entered ( $W 9$ and $W 10$ ).

W12(R) JUNCTION HYORAULIC DIAMETER ( $\mathrm{m}, \mathrm{ft})$. This word is optional and is not used in SCDAP/RELAP5/MOD2.5 but is used in SCDAP/RELAP5/MOD3. Default value is zero. If zero is entered, the hydraulic diameter is set to four times the junction area divided by the wetted perimeter.

\subsection{Cards ccc6nnN, Volume Initial Condition Data Cards}

These cards are required. NNN ranges from 001 through 999 and need not be consecutive.

These cards use the first six words as overlay information to specify the range of volumes for which the following initial condition information applies. Each volume must be referenced at least once by these cards. 
W1(I) $\quad X 1$

W2(I) $\quad \times 2$

W3(I) YI

W4(I) Y2

W5(I) $\quad \mathrm{Zl}$

W6(I) Z2

W7(I) CONTROL WORD. This word has the packed format $\underline{\underline{\mathrm{bt}}}$.

The digit $\epsilon$ specifies the fluid. $\epsilon=0$ is the default fluid. $\underline{\epsilon}=1$ specifies water, $\underline{\epsilon}=2$ specifies $D 20$, and $\underline{\epsilon}=3$ specifies hydrogen. The default fluid is that set for the hydrodynamic system by Cards 120 through 129 or this control word in another volume in this hydrodynamic system. The fluid type set on Cards 120 through 129 or these control words must be consistent (i.e., not specify different fluids). If Cards 120 through 129 are not entered and all control words use the default $\underline{\epsilon}=0$, then water is assumed as the fluid.

The digit $\underline{b}$ specifies whether boron is present or not. The digit $\underline{b}=0$ specifies that the volume fluid does not contain boron; $\underline{b}=1$ specifies that a boron concentration in parts of boron per parts of liquid water (which may be zero) is being entered after the other required thermodynamic information.

The digit $\underline{t}$ specifies how the following words are to be used to determine the initial thermodynamic state. Entering $\underline{t}=0$ through 3 specifies only one component (steam/water). Entering $t=4$ through 6 allows the specification of two components (steam/water and noncondensable gas). 
If $\underline{t=0}$, the next four words are interpreted as pressure $\left(\mathrm{Pa}, 1 \mathrm{~b}_{\mathrm{f}} / \mathrm{in}^{2}\right)$, liquid specific internal energy $(\mathrm{J} / \mathrm{kg}, \mathrm{Btu} / \mathrm{lb})$, and vapor void fraction; these quantities will be interpreted as nonequilibrium or equilibrium conditions depending on the volume control flag. If equilibrium, the static quality is checked; but only the nressure and internal energies are used to define the thermodynamic state.

If $t=1$, the next two words are interpreted as temperature $\left(K,{ }^{\circ} \mathrm{F}\right)$ and quality in equilibrium condition.

If $\underline{t=2}$, the next two words are interpreted as pressure $\left(\mathrm{Pa}, 1 \mathrm{~b}_{\mathrm{f}} / \mathrm{in}^{2}\right)$ and quality in equilibrium condition.

If $t=3$, the next two words are interpreted as pressure $\left(\mathrm{Pa}, 1 \mathrm{~b}_{\mathrm{f}} / \mathrm{in}^{2}\right)$ and temperature $\left(\mathrm{K},{ }^{\circ} \mathrm{F}\right)$ in equilibrium condition.

If $t=4$, the next three words are interpreted as pressure $\left(\mathrm{Pa}, 1 \mathrm{~b}_{\mathrm{f}} / \mathrm{in}^{2}\right)$, temperature $\left(\mathrm{K},{ }^{\circ} \mathrm{F}\right)$, and equilibrium quality. This value of $t=4$ is for input of a noncondensable equilibrium state. The equilibrium quality must be $\geq 0$ and $\leq 1$.

If $\underline{t=5}$, the next three words are interpreted as temperature $\left(K,{ }^{\circ} \mathrm{F}\right)$, equilibrium quality, and noncondensable quality. The equilibrium and noncondensable quality must be $\geq 0$ and $\leq 1$.

If $\underline{t=6}$, the next five words are interpreted as pressure $\left(\mathrm{Pa}, 1 \mathrm{~b}_{\mathrm{f}} / \mathrm{in}^{2}\right)$, liquid specific internal energy $(\mathrm{J} / \mathrm{kg}, \mathrm{Btu} / \mathrm{lb})$, vapor specific internal energy $(\mathrm{J} / \mathrm{kg}, \mathrm{Btu} / \mathrm{lb})$, vapor void fraction, and noncondensable quality. The vapor void fraction and noncondensable quality must be $\geq 0$ and $\leq 1$.

W8-13(R) INITIAL CONDITION DATA, Depending on the control word, two through five quantities may be required. Enter only the minimum number required. If entered, boron concentration follows the last required word for thermodynamic conditions. 


\subsection{CaRdS CCC7001 Through CCC9999, Junction Initial Condition Data Cards}

These cards are required. The range of card numbers need not be consecutive.

These cards use the first six words as overlay information to specify a range of volumes and in addition use a seventh number to specify the volume face. The information following the face number is applied to the junction originating from the specified face of each volume included in the overlay. All junctions must be referenced at least once by these cards.

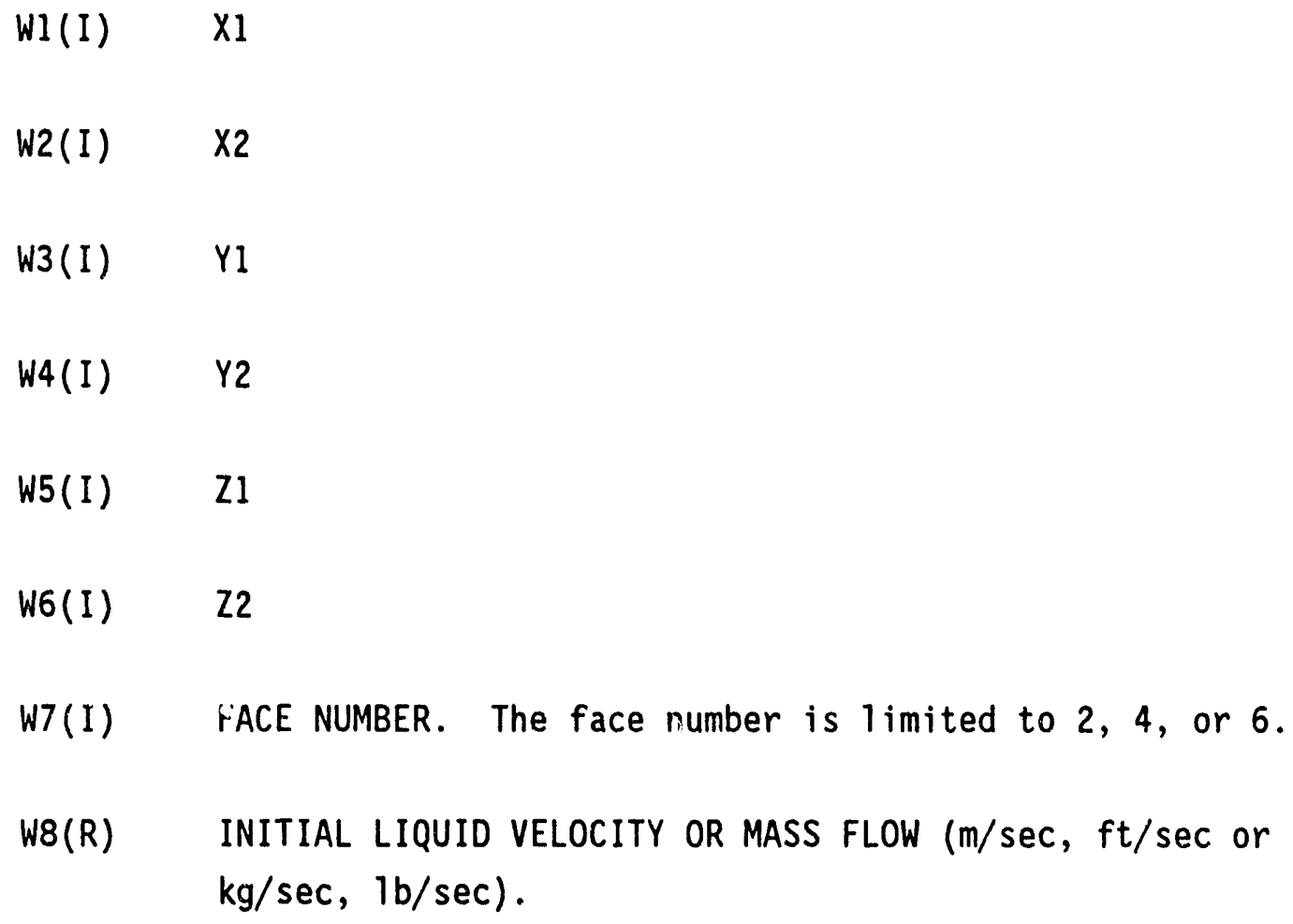

W9(R) INITIAL VAPOR VELOCITY OR MASS FLOW $(\mathrm{m} / \mathrm{sec}, \mathrm{ft} / \mathrm{sec}$ or $\mathrm{kg} / \mathrm{sec}, \mathrm{lb} / \mathrm{sec})$. 


\subsection{MJDELING GUIDELINES AND ASSUMPTIONS}

The multidimensional component has only been operational in RELAP5/MOD2.5 for a short period of time. As a result, there has been limited use of the component to date; however, in the future, its use is expected to increase. With increased usage of the component, more modeling guidelines will be developed. The following guidelines are those that have been developed to date:

1. As indicated in Section 4.5, tho interphase heat transfer coefficients in the 3 directions are weighted by the volume hydraulic diameters in the 3 directions. For water to fall evenly as in the fall problem, the following is required: At a given level, the diameters in a given direction must all be the same value, although they need not be the same as the other 2 direction's hydraulic diameters at this level. Otherwise, the code will calculate different interphase heat transfer coefficients in volumes at a given level, which then results in a different void fraction in volumes at a given level.

2. The 3-D component can be connected to other components externally via either a normal junction or a crossflow junction, depending on the actual flow paths.

3. The 3-D cylindrical component can be modeled either as a solid cylinder or a hollow cylinder. It can also be modeled as a cylindrical wedge. All of these geometries are implemented.

4. The volume factors and junction areas factors must be specified explicitly for the 3-D component.

5. In the junction initial condition cards, the junction face number must be specified explicitly.

6. For pure radial, frictionless flow in and out of a 3-D solid cylinder, the pressure profile within the 3-D component is not sensitive to the number of radial nodes, i.e., a 3-ring model 
produced as good pressure results as an 8-ring model. The user does not have to increase radial nodes to increase accuracy.

7. The 1-D-to-3-D connections appear to exhibit some froblems as evidenced by the problems seen in the external connections to the inner ring of the multidimensional component. Further development and assessment is required to resolve this. 

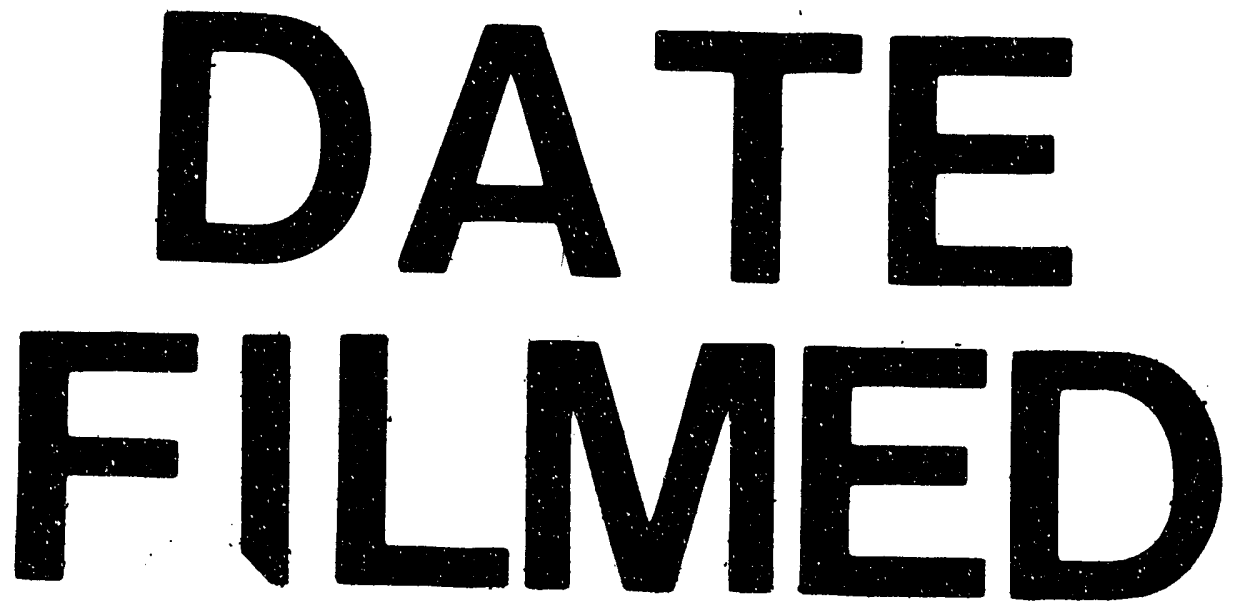

01108193 


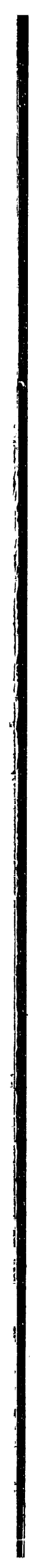

